\title{
THE CHANDRA DEEP FIELD-SOUTH SURVEY: 7 MS SOURCE CATALOGS
}

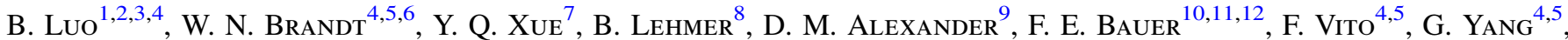 \\ A. R. Basu-ZYCH ${ }^{13,14}$, A. Comastri ${ }^{15}$, R. Gilli ${ }^{15}$, Q.-S. Gu ${ }^{1,2,3}$, A. E. Hornschemeier ${ }^{13}$, A. KoeKemoer ${ }^{16}$, T. LiU $^{7}$,

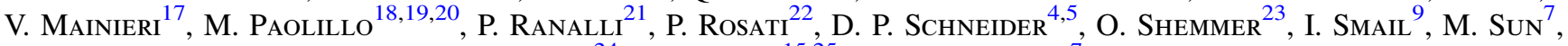 \\ P. Tozzi ${ }^{24}$, C. Vignali ${ }^{15,25}$, AND J.-X. WANG ${ }^{7}$ \\ ${ }^{1}$ School of Astronomy and Space Science, Nanjing University, Nanjing, Jiangsu 210093, China \\ ${ }^{2}$ Key Laboratory of Modern Astronomy and Astrophysics (Nanjing University), Ministry of Education, Nanjing, Jiangsu 210093, China \\ ${ }^{3}$ Collaborative Innovation Center of Modern Astronomy and Space Exploration, Nanjing, Jiangsu 210093, China \\ ${ }^{4}$ Department of Astronomy \& Astrophysics, 525 Davey Lab, The Pennsylvania State University, University Park, PA 16802, USA \\ ${ }^{5}$ Institute for Gravitation and the Cosmos, The Pennsylvania State University, University Park, PA 16802, USA \\ ${ }^{6}$ Department of Physics, 104 Davey Lab, The Pennsylvania State University, University Park, PA 16802, USA \\ ${ }^{7}$ CAS Key Laboratory for Research in Galaxies and Cosmology, Department of Astronomy, \\ University of Science and Technology of China, Hefei, Anhui 230026, China \\ ${ }^{8}$ Department of Physics, University of Arkansas, 226 Physics Building, 835 West Dickson Street, Fayetteville, AR 72701, USA \\ ${ }^{9}$ Centre for Extragalactic Astronomy, Department of Physics, Durham University, Durham DH1 3LE, UK \\ ${ }^{10}$ Instituto de Astrofísica, Facultad de Física, Pontificia Universidad Católica de Chile, 306, Santiago 22, Chile \\ ${ }^{11}$ Millennium Institute of Astrophysics (MAS), Nuncio Monseñor Sótero Sanz 100, Providencia, Santiago, Chile \\ ${ }^{12}$ Space Science Institute, 4750 Walnut Street, Suite 205, Boulder, CO 80301, USA \\ ${ }^{13}$ NASA Goddard Space Flight Center, Code 662, Greenbelt, MD 20771, USA \\ 14 Department of Physics, University of Maryland Baltimore County, Baltimore, MD 21250, USA \\ ${ }_{15}^{5}$ INAF-Osservatorio Astronomico di Bologna, via Ranzani 1, I-40127 Bologna, Italy \\ ${ }^{16}$ Space Telescope Science Institute, 3700 San Martin Drive, Baltimore, MD 21218, USA \\ ${ }^{17}$ European Southern Observatory, Karl-Schwarzschild-Strasse 2, D-85748, Garching bei München, Germany \\ ${ }^{18}$ Dip.di Fisica Ettore Pancini, University of Naples "Federico II", C.U. Monte S. Angelo, Via Cintia, I-80126, Naples, Italy \\ ${ }^{19}$ INFN-Sez.di Napoli, Via Cintia, I-80126, Naples, Italy \\ ${ }^{20}$ Agenzia Spaziale Italiana-Science Data Center, Via del Politecnico snc, I-00133 Roma, Italy \\ ${ }^{21}$ Lund Observatory, Department of Astronomy and Theoretical Physics, Lund University, Box 43, SE-22100 Lund, Sweden \\ ${ }^{22}$ Dipartimento di Fisica e Scienze della Terra, Università degli Studi di Ferrara, Via Saragat 1, I-44122 Ferrara, Italy \\ ${ }^{23}$ Department of Physics, University of North Texas, Denton, TX 76203, USA \\ ${ }^{24}$ INAF-Osservatorio Astrofisico di Arcetri, Largo E. Fermi 5, I-50125, Florence, Italy \\ ${ }^{25}$ Dipartimento di Fisica e Astronomia, Alma Mater Studiorum, Università degli Studi di Bologna, Viale Berti Pichat 6/2, I-40127 Bologna, Italy \\ Received 2016 September 17; revised 2016 October 31; accepted 2016 November 7; published 2016 December 27
}

\begin{abstract}
We present X-ray source catalogs for the $\approx 7$ Ms exposure of the Chandra Deep Field-South (CDF-S), which covers a total area of $484.2 \mathrm{arcmin}^{2}$. Utilizing WAVDETECT for initial source detection and ACIS Extract for photometric extraction and significance assessment, we create a main source catalog containing 1008 sources that are detected in up to three X-ray bands: $0.5-7.0 \mathrm{keV}, 0.5-2.0 \mathrm{keV}$, and 2-7 keV. A supplementary source catalog is also provided, including 47 lower-significance sources that have bright $\left(K_{s} \leqslant 23\right)$ near-infrared counterparts. We identify multiwavelength counterparts for 992 (98.4\%) of the main-catalog sources, and we collect redshifts for 986 of these sources, including 653 spectroscopic redshifts and 333 photometric redshifts. Based on the X-ray and multiwavelength properties, we identify 711 active galactic nuclei (AGNs) from the main-catalog sources. Compared to the previous $\approx 4 \mathrm{Ms}$ CDF-S catalogs, 291 of the main-catalog sources are new detections. We have achieved unprecedented X-ray sensitivity with average flux limits over the central $\approx 1 \operatorname{arcmin}^{2}$ region of $\approx 1.9 \times 10^{-17}, 6.4 \times 10^{-18}$, and $2.7 \times 10^{-17} \mathrm{erg} \mathrm{cm}^{-2} \mathrm{~s}^{-1}$ in the three X-ray bands, respectively. We provide cumulative number-count measurements observing, for the first time, that normal galaxies start to dominate the $\mathrm{X}$-ray source population at the faintest $0.5-2.0 \mathrm{keV}$ flux levels. The highest X-ray source density reaches $\approx 50,500$ $\mathrm{deg}^{-2}$, and $47 \% \pm 4 \%$ of these sources are AGNs $\left(\approx 23,900 \mathrm{deg}^{-2}\right)$.
\end{abstract}

Key words: catalogs - cosmology: observations - diffuse radiation - galaxies: active - surveys - X-rays: galaxies

Supporting material: extended figures, machine-readable tables

\section{INTRODUCTION}

Cosmic X-ray surveys of the distant universe have made enormous advances over the past two decades, since the launches of the Chandra X-ray Observatory (Chandra; e.g., Weisskopf et al. 2000) and X-Ray Multi-Mirror Mission (XMM-Newton; e.g., Jansen et al. 2001). These surveys are a primary source of information about accreting supermassive black holes (SMBHs), i.e., active galactic nuclei (AGNs), in the universe, providing insights about their demographics, physical properties, and interactions with their environments (e.g.,
Brandt \& Alexander 2015 and references therein). Furthermore, X-ray surveys are an essential tool for the study of clusters and groups (e.g., Allen et al. 2011 and references therein), as well as X-ray binary populations in starburst and normal galaxies (e.g., Mineo et al. 2014; Lehmer et al. 2016; and references therein). X-ray surveys with a wide variety of sensitivities and solid angles are required to gain a comprehensive understanding of X-ray source populations in the universe (e.g., see Section 2.1 of Brandt \& Alexander 2015). Such surveys range from shallow, all-sky surveys, the lowest tier of the X-ray surveys "wedding cake," to the highest tier of 
ultra-deep, pencil-beam surveys. Ultra-deep X-ray surveys are particularly important as cosmic "time machines," since fainter X-ray sources of a given type generally lie at higher redshifts and thus earlier cosmic epochs. Furthermore, at a given redshift, such ultra-deep surveys are capable of probing objects with lower observable X-ray luminosities that are generally more typical members of source populations. Additionally, some intrinsically luminous X-ray sources may have low observable X-ray luminosities owing to strong obscuration (e.g., Compton-thick AGNs), and ultra-deep X-ray surveys are one of the key ways of identifying and characterizing such important sources (e.g., see Section 3.3 of Brandt \& Alexander 2015).

The deepest X-ray surveys to date have been conducted in the Chandra Deep Field-South (CDF-S), which is arguably the most intensively studied multiwavelength deep-survey region across the entire sky. Currently, published CDF-S X-ray catalogs exist for the $4 \mathrm{Ms}$ Chandra exposure (covering $465 \mathrm{arcmin}^{2}$; e.g., Xue et al. 2011) and the 3 Ms XMM-Newton exposure (covering $830 \mathrm{arcmin}^{2}$; e.g., Ranalli et al. 2013). In 2013, we proposed to extend the 4 Ms CDF-S observations to a total Chandra exposure of $7 \mathrm{Ms}$, and the new observations were conducted during 2014-2016. The very small Chandra point-spread function (PSF) and low background still allow for significant gains in sensitivity near the field center, and thus the detection of many new sources, even for such long exposures. Furthermore, all previously detected CDF-S sources benefit greatly from the improved photon statistics, which allow better measurements of X-ray positions, photometric properties, spectral properties, and variability; variability studies also benefit from the significantly lengthened time baseline of sensitive CDF-S X-ray observations (e.g., Yang et al. 2016). These better X-ray measurements advance significantly the physical understanding of the sources producing most of cosmic accretion power; e.g., a typical AGN in the CDF-S will have $\approx 40$ times more counts than the same AGN in the COSMOS Legacy Survey (e.g., Civano et al. 2016).

In this paper, we will present CDF-S point-source catalogs derived from the $7 \mathrm{Ms}$ Chandra exposure for use by the community in advancing X-ray deep-survey science projects; previous Chandra Deep Fields catalogs of this type have been widely utilized (e.g., Alexander et al. 2003; Luo et al. 2008; Xue et al. 2011, 2016). We will also present multiwavelength identifications, basic multiwavelength photometry, and spectroscopic/photometric redshifts for the detected X-ray sources. Chandra source-cataloging methodology has advanced greatly over the years since the Chandra launch, providing substantially improved yields of demonstrably reliable sources (e.g., Xue et al. 2016) and improved source characterization. Here we will utilize ACIS Extract (AE; Broos et al. 2010) ${ }^{26}$ as a central part of our point-source cataloging. AE is used as part of an effective two-stage source-detection approach, and it allows for the optimal combination of multiple observations with different aim points and roll angles.

Some key AGN science projects that should be advanced by the $7 \mathrm{Ms}$ CDF-S include investigations of (1) how SMBHs, including those in obscured systems, grow and co-evolve with galaxies through the critical era at $z \approx 1-4$ when massive galaxies were largely assembling, and (2) how SMBHs grow within the first galaxies at $z>4$. Starburst and normal galaxies

\footnotetext{
26 See http://www.astro.psu.edu/xray/docs/TARA/ae_users_guide.html for details on ACIS Extract.
}

are also detected in abundance at the faintest X-ray fluxes; their differential number counts are comparable to those of AGNs at the faintest $0.5-2.0 \mathrm{keV}$ fluxes reached by the $4 \mathrm{Ms}$ CDF-S (e.g., Lehmer et al. 2012). The 7 Ms CDF-S will thus be a key resource for examining how the X-ray binary populations of starburst and normal galaxies evolve over most of cosmic time, both via studies of the directly detected sources and via stacking analyses (e.g., Lehmer et al. 2016; Vito et al. 2016). Owing to its unique combination of great depth and high angular resolution, the $7 \mathrm{Ms}$ CDF-S should serve as a multidecade Chandra legacy. For example, even Athena, a nextgeneration X-ray observatory aiming for launch in $\approx 2028$ (e.g., Barcons et al. 2015), may not be able to reach the flux levels probed in the central region of the $7 \mathrm{Ms}$ CDF-S. X-ray missions capable of substantially surpassing the sensitivity of the $7 \mathrm{Ms}$ CDF-S, such as the X-Ray Surveyor (e.g., Weisskopf et al. 2015), are presently not funded for construction.

The structure of this paper is as follows. In Section 2, we present the new Chandra observations and the reduction details for the full data set. Section 3 describes the creation of observation images, exposure maps, and the main and supplementary source catalogs. In Section 4, we present the main Chandra source catalog in detail. Here, we also present key aspects of the X-ray source characterization and the multiwavelength identifications. We briefly compare the properties of the newly detected sources to those already found in the $4 \mathrm{Ms}$ CDF-S. Section 5 presents the supplementary catalog of X-ray sources that have lower detection significances, but align spatially with bright near-infrared (NIR) sources. In Section 6, we present an analysis of source completeness and reliability, showing that we strike a reasonable balance between these two criteria. Section 7 describes an analysis of the background and sensitivity across the CDF-S. In Section 8, we present cumulative number counts for the main Chandra source catalog, and in Section 9, we provide a summary of the main results. The Chandra source catalogs and several associated data products are being made publicly available along with this paper. ${ }^{27}$

We adopt a Galactic column density of $N_{\mathrm{H}}=8.8 \times 10^{19}$ $\mathrm{cm}^{-2}$ along the line of sight to the CDF-S (e.g., Stark et al. 1992). Coordinates are presented in the J2000.0 system, and magnitudes are in the AB system (Oke \& Gunn 1983). We quote uncertainties at a $1 \sigma$ confidence level and upper/lower limits at a $90 \%$ confidence level. A cosmology with $H_{0}=67.8$ $\mathrm{km} \mathrm{s}^{-1} \mathrm{Mpc}^{-1}, \Omega_{\mathrm{M}}=0.308$, and $\Omega_{\Lambda}=0.692$ (Planck Collaboration et al. 2016) is used.

\section{OBSERVATIONS AND DATA REDUCTION}

\subsection{Observations of the $7 \mathrm{Ms} C D F-S$}

The $7 \mathrm{Ms}$ CDF-S contains observations taken in four separate epochs of time. The basic information on these observations, 102 in total, is listed in Table 1. There were 48 recent observations acquired between 2014 June 9 and 2016 March 24, which constitute the last $3 \mathrm{Ms}$ of exposure of the $7 \mathrm{Ms}$ CDF-S. The first $1 \mathrm{Ms}$ of exposure consists of 11 observations taken between 1999 and 2000 (Giacconi et al. 2002; Rosati et al. 2002; Alexander et al. 2003), the next $1 \mathrm{Ms}$ of exposure consists of 12 observations taken in

\footnotetext{
${ }^{27}$ http://www.astro.psu.edu/users/niel/cdfs/cdfs-chandra.html.
} 
Table 1

Journal of 7 Ms Chandra Deep Field-South Observations

\begin{tabular}{|c|c|c|c|c|c|c|c|}
\hline \multirow{2}{*}{ Obs. ID } & \multirow{2}{*}{$\begin{array}{l}\text { Obs. Start } \\
\text { (UT) }\end{array}$} & \multirow{2}{*}{$\begin{array}{l}\text { Exposure } \\
\text { Time (ks) }\end{array}$} & \multicolumn{2}{|c|}{ Aim Point } & \multirow{2}{*}{$\begin{array}{l}\text { Roll Angle } \\
\quad \text { (deg) }\end{array}$} & \multirow{2}{*}{$\begin{array}{l}\text { Obs. } \\
\text { Mode }\end{array}$} & \multirow{2}{*}{$\begin{array}{l}\text { Pipeline } \\
\text { Version }\end{array}$} \\
\hline & & & $\alpha(\mathrm{J} 2000.0)$ & $\delta(\mathrm{J} 2000.0)$ & & & \\
\hline $1431-0$ & 1999 Oct $15,17: 38$ & 25.1 & 033229.31 & -274822.2 & 47.3 & $\mathrm{VF}$ & 8.4 .5 \\
\hline 441 & 2000 May 27, 01:18 & 53.5 & 033226.91 & $-2748 \quad 19.4$ & 166.7 & $\mathrm{~F}$ & 8.4 .5 \\
\hline 582 & 2000 Jun $03,02: 38$ & 127.6 & 033226.97 & -274818.5 & 162.9 & $\mathrm{~F}$ & 8.4 .5 \\
\hline 2406 & 2000 Dec $10,23: 35$ & 28.7 & 033228.33 & -274836.5 & 332.2 & $\mathrm{~F}$ & 8.4 .5 \\
\hline 2405 & 2000 Dec $11,08: 14$ & 56.3 & 033228.82 & -274843.5 & 331.8 & $\mathrm{~F}$ & 8.4 .5 \\
\hline
\end{tabular}

Note. The 7 Ms CDF-S consists of 102 observations, with a total cleaned exposure time of 6.727 Ms. The average aim point for the merged observations, weighted by the individual exposure times, is $\alpha_{\mathrm{J} 2000.0}=03^{\mathrm{h}} 32^{\mathrm{m}} 28^{\mathrm{s}} .27, \delta_{\mathrm{J} 2000.0}=-27^{\circ} 48^{\prime} 21^{\prime \prime}$. 8 . The observations were performed using Faint (F) or Very Faint (VF) modes.

(This table is available in its entirety in machine-readable form.)

2007 (Luo et al. 2008), and another 2 Ms of exposure includes 31 observations in 2010 (Xue et al. 2011).

All 102 CDF-S observations used the Chandra Advanced CCD Imaging Spectrometer imaging array (ACIS-I; Garmire et al. 2003), which is optimized for Chandra surveys. ACIS-I consists of four CCDs (I0-I3) with $1024 \times 1024$ pixels each; the size of the CCD pixels is 0 ". $492 \times 0$ " 492 , and the ACIS-I array size or the field of view of each observation is $16 ! 9 \times$ $16 ! 9=285 \operatorname{arcmin}^{2}$. Of the 11 observations in the first $1 \mathrm{Ms}$ of CDF-S exposure, 10 (except observation 1431-0) were taken in Faint mode, while the first observation (1431-0) and all the observations in the later $6 \mathrm{Ms}$ of CDF-S exposure were taken in Very Faint mode (see Table 1), which reduces ACIS-I particle background significantly and improves detection of weak sources (Vikhlinin 2001). During the first two observations (1431-0 and 1431-1), the nominal focal-plane temperature was $-110^{\circ} \mathrm{C}$, while it was $-120^{\circ} \mathrm{C}$ for the other observations.

The roll angles of the 102 observations (Table 1) were intentionally allowed to vary, in order to obtain more uniform sensitivity across the field by averaging out some of the CCDgap effects (e.g., see Figure 1 below) and to obtain a larger areal coverage. The total area covered by the $7 \mathrm{Ms}$ CDF-S is $484.2 \operatorname{arcmin}^{2}$, substantially larger than the field of view of ACIS-I. The aim points of individual observations also differ slightly (Table 1); the average aim point for the merged observations, weighted by the individual exposure times, is $\alpha_{\mathrm{J} 2000.0}=03^{\mathrm{h}} 32^{\mathrm{m}} 28^{\mathrm{s}} .27, \delta_{\mathrm{J} 2000.0}=-27^{\circ} 48^{\prime} 21^{\prime \prime} 8$.

\subsection{Data Reduction}

The data for the 102 observations downloaded from the Chandra X-ray Center (CXC) have gone through the CXC pipeline software for basic processing. The software versions are listed in Table 1, and the data for the previous 4 Ms CDF-S observations have been processed with newer versions of the software compared to those presented in the previous catalog papers (Luo et al. 2008; Xue et al. 2011). These data were then reduced and analyzed utilizing Chandra Interactive Analysis of Observations (CIAO; v4.8) ${ }^{28}$ tools, AE (version 2016may25), the MARX ray-tracing simulator (v5.3) that is used in AE to model the Chandra ACIS-I PSF, ${ }^{29}$ and custom software. Most

\footnotetext{
${ }^{28}$ See http://cxc.harvard.edu/ciao/ for details on CIAO.

29 http://space.mit.edu/CXC/MARX/; this version of MARX fixed a PSF issue affecting the PSF simulations of off-axis sources (see https://github. com/Chandra-MARX/marx/pull/21).
}

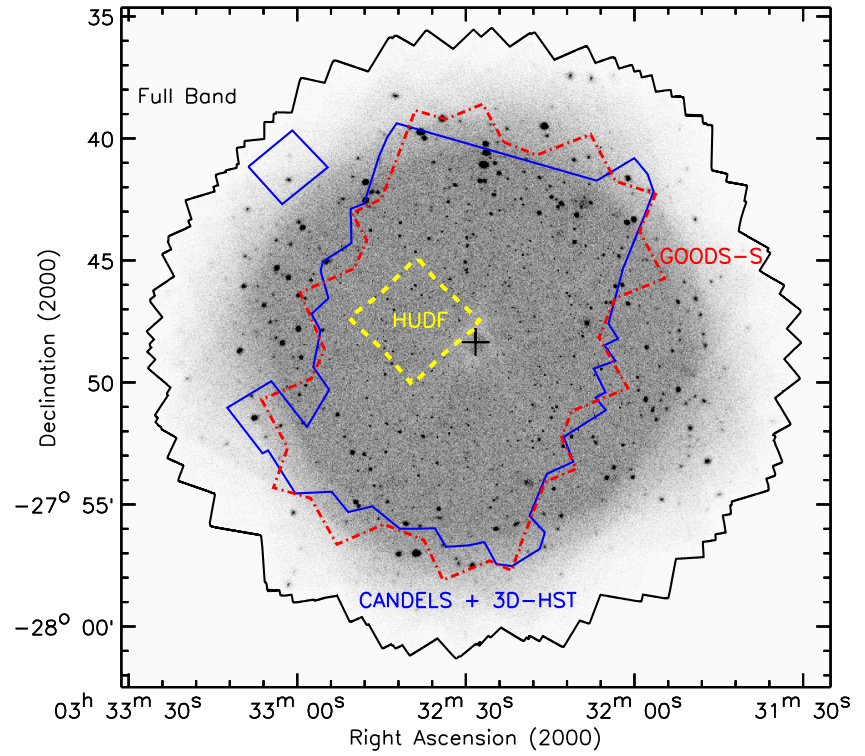

Figure 1. Full-band raw image of the $7 \mathrm{Ms}$ CDF-S in linear gray scale. The black outline surrounding the image indicates the coverage of the entire CDF$\mathrm{S}$. The blue solid, red dash-dotted, and yellow dashed regions show the coverage of the CANDELS + 3D-HST fields, GOODS-S survey, and HUDF, respectively. The central black plus sign marks the average aim point. The apparent lightening of the area surrounding the black plus sign is caused by the relatively low effective exposure in this region due to the ACIS-I CCD gaps (see Figure 2). The apparent scarcity of sources near the field center is largely due to the small PSF size at that location, which makes sources difficult to identify visually in this figure (see Figures 4 and 18 for clarification).

of the procedures are similar to those performed in Luo et al. (2008) and Xue et al. (2011, 2016), and the main steps are described below.

We adopted the CIAO tool ACIS_PROCESS_EVENTS to reprocess level 1 event files, applying Charge Transfer Inefficiency (CTI) corrections for observations with nominal focal-plane temperatures of $-120^{\circ} \mathrm{C}$ (Townsley et al. 2000, 2002), flagging potential cosmic-ray background events for Very Faint mode data (CHECK_VF_PHA=YES), and implementing a custom strippeddown bad-pixel file instead of the standard CXC one. A large fraction of the bad-pixel locations in the standard bad-pixel file appear to contain good $>0.7 \mathrm{keV}$ events that are appropriate for source searching and characterization; instead of rejecting all events falling on these pixels, we chose to exclude manually those events below a row-dependent energy of $0.5-0.7 \mathrm{keV}$ that fall on 
"hot" soft columns (see Section 2.2 of Luo et al. 2008 for details). This approach allows us to recover a significant number of additional good events $(\approx 3.2 \%$ of the total) compared to the standard level 2 data products from the CXC pipeline.

We employed the CIAO tool ACIS_DETECT_AFTERGLOW to identify and flag cosmic-ray afterglow events. Following the procedure of Luo et al. (2008) and Xue et al. (2011), we further employed custom software and removed 113 additional faint afterglow events with three or more total counts falling on the same CCD pixel within 20 s (see Footnote 27 of Xue et al. 2011 for details about this choice). We inspected the background light curve of each observation utilizing the EVENT BROWSER tool in the Tools for ACIS Review \& Analysis (TARA; Broos et al. 2000), and we removed background flares using the CIAO tool DEFLARE with an iterative $3 \sigma$ clipping approach. The $7 \mathrm{Ms}$ CDF-S observations are not significantly affected by background flares. Only four observations (1431-0, 16176, 16184, 17542) were affected by flares longer than $10 \%$ of their durations (up to $\approx 15 \%$ ), while the other observations have milder or no background flares. The cleaned exposure time for each observation is listed in Table 1. In total, $1.2 \%$ of the exposure was removed due to background flares; the total cleaned exposure is 6.727 Ms.

After generating a cleaned event file for each of the 102 observations, the next steps were to register and align these observations to a common astrometric frame and merge them into a combined master event file. The first action was fixing any astrometric offsets of individual observations. For each observation, we created a $0.5-7.0 \mathrm{keV}$ image using the standard ASCA grade set (grades $0,2,3,4,5$ ). We then searched for $\mathrm{X}$-ray sources in the image using WAVDETECT (Freeman et al. 2002) with a " $\sqrt{2}$ sequence" of wavelet scales (i.e., 1, $1.414,2,2.828,4,5.656$, and 8 pixels) and a false-positive probability threshold of $10^{-6}$. A PSF-size image was supplied to the WAVDETECT run that was created with the CIAO tool MKPSFMAP assuming a power-law spectrum with a photon index of $\Gamma=1.4$ and an enclosed counts fraction (ECF) of 0.4 . Depending on the exposure times of the observations, $\approx 40-170$ $\mathrm{X}$-ray sources are detected in the individual data sets. The initial X-ray positions of these WAVDETECT sources were refined using $\mathrm{AE}$, and the centroid positions determined by $\mathrm{AE}$ from its "CHECK_POSITIONS" stage were adopted as the improved positions of these sources. We registered the absolute astrometry of each observation to a common frame by matching the X-ray source positions to the NIR source positions in the Taiwan ECDFS Near-Infrared Survey (TENIS) $K_{s}$-band catalog (Hsieh et al. 2012), where 6651 bright $\left(K_{s} \leqslant 22\right)$ TENIS sources within the field of view of the $7 \mathrm{Ms}$ CDF-S were used. This NIR catalog was chosen as its astrometric frame is consistent with those of other optical/ NIR catalogs (e.g., see the list in Section 4.2 below) ${ }^{30}$ and the

\footnotetext{
$\overline{30}$ The astrometry of the Xue et al. (2011) 4 Ms CDF-S observations was registered to the frame of the Very Large Array catalog of Miller et al. (2013), and there are $\approx 0$.' 2 offsets in right ascension and declination between this frame and those of the optical/NIR catalogs, median $\left(\mathrm{RA}_{\mathrm{TENIS}}-\mathrm{RA}_{\mathrm{VLA}}\right)=$

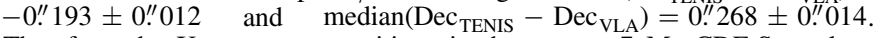
Therefore, the X-ray source positions in the current $7 \mathrm{Ms}$ CDF-S catalogs have the same systematic offsets from the $4 \mathrm{Ms}$ source positions inherited from the different choices of the astrometric systems. We caution that the TENIS astrometric frame is off from that of the first Gaia data release (Gaia Collaboration et al. 2016) by median $\left(\mathrm{RA}_{\mathrm{TENIS}}-\mathrm{RA}_{\mathrm{Gaia}}\right)=$ -0 ". $154 \pm 0$ ". 004 and median(Dec TENIS $-\operatorname{Dec}_{\text {Gaia }}$ ) $=0$ ". $290 \pm 0$ ". 005. The Miller et al. (2013) Very Large Array astrometric frame thus agrees better with the Gaia frame, although there are not enough sources in common for a direct comparison.
}

fractions of X-ray sources with a bright NIR counterpart are high (ranging from $\approx 50 \%-80 \%$ ) in the CDF-S observations.

The matching of the X-ray and TENIS sources as well as the World Coordinate System (WCS) update were performed using the CIAO tool REPROJECT_ASPECT with a $3^{\prime \prime}$ matching radius and a 0 ". 6 residual limit; ${ }^{31} \approx 30-110$ matches are found in the individual observations, and the positional offsets were used to obtain the WCS transformation for each observation. The WCS transformation matrices range from 0 ".007 to 1 ".011 in linear translation, -0.051 to 0.014 in rotation, and 0.9996 to 1.0012 in scaling; the resulting registrations are accurate to $\approx 0$ ". 3 . Although the astrometric offsets are small, registering the observations and correcting for the offsets is a necessary step for detecting very faint X-ray sources and obtaining the bestpossible X-ray source positions as well as reliable photometric properties. The astrometry registered event files were produced with the CIAO tool REPROJECT_EVENTS. We then projected the event files to the astrometric frame of observation $2406^{32}$ using REPROJECT_ASPECT and REPROJECT_EVENTS. Finally, we merged these 102 event files into a master event file using the CIAO tool DMMERGE. We note that X-ray source detection was performed in the merged observation while source characterization was carried out mainly using the individual astrometry registered observations (e.g., the AE photometric extraction).

\section{IMAGES, EXPOSURE MAPS, AND X-RAY SOURCE DETECTION}

\subsection{Image and Exposure Map Creation}

We created X-ray images from the merged event file using the standard ASCA grade set in three bands: $0.5-7.0 \mathrm{keV}$ (full band; FB), 0.5-2.0 keV (soft band; SB), and 2-7 keV (hard band; HB). ${ }^{33}$ The full-band raw image is shown in Figure 1; also illustrated are the fields of view of some of the deepest optical-NIR surveys from the Hubble Space Telescope (HST) within the CDF-S, including the Hubble Ultra Deep Field (HUDF; Beckwith et al. 2006), the Great Observatories Origins Survey Southern field (GOODS-S; Giavalisco et al. 2004), and the combined field of the Cosmic Assembly Near-infrared Deep Extragalactic Legacy Survey (CANDELS; Grogin et al. 2011; Koekemoer et al. 2011) and the 3D-HST survey (Skelton et al. 2014).

Due to the effects of vignetting, gaps between the CCDs, bad-pixel filtering, bad-column filtering, spatially dependent degradation in quantum efficiency due to contamination on the ACIS optical-blocking filters, and slightly different aim points between observations, the effective exposure time of the combined observation cannot reach the total cleaned exposure of $6.727 \mathrm{Ms}$, and it varies across the CDF-S field. Therefore, we constructed effective-exposure maps in the three bands following the basic approach described in Section 3.2 of Hornschemeier et al. (2001), taking into account the above effects. A power-law spectrum with a photon index of $\Gamma=1.4$

\footnotetext{
31 This is a parameter used in REPROJECT_ASPECT to remove source pairs with residual positional offsets larger than the given limit.

${ }^{32}$ The choice of this astrometric frame is to be consistent with our previous CDF-S analyses (Luo et al. 2008; Xue et al. 2011); choosing the frame of another observation does not affect any of the results.

33 The upper energy bound of the bands has been changed from $8 \mathrm{keV}$ in our previous CDF-S analyses (Alexander et al. 2003; Luo et al. 2008; Xue et al. 2011) to $7 \mathrm{keV}$; see Footnote 16 of Xue et al. (2016) for detailed reasoning.
} 


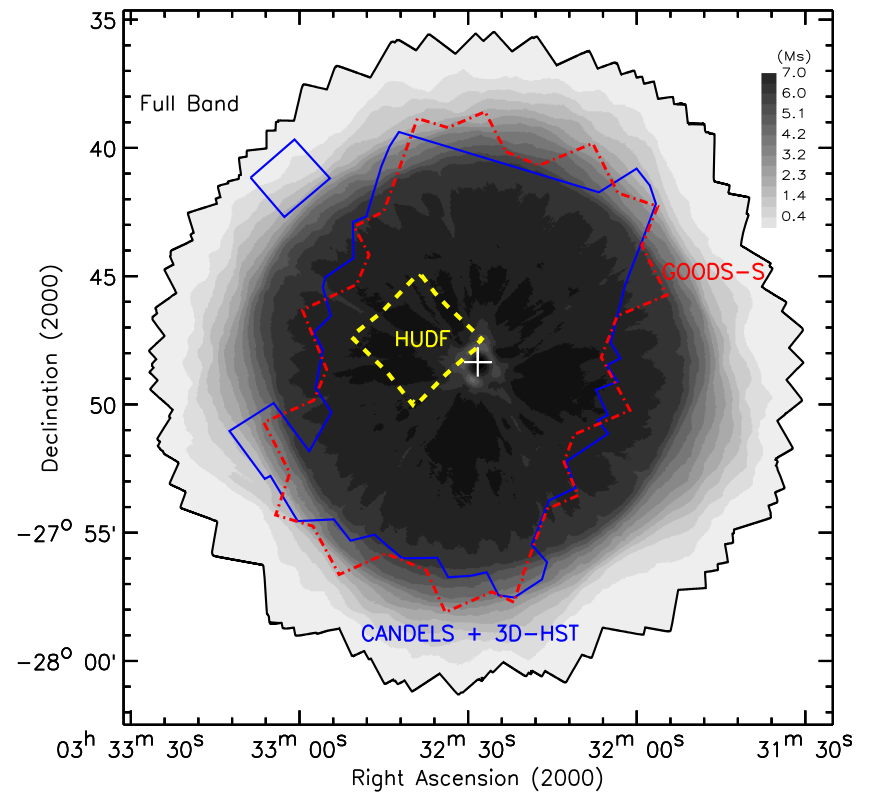

Figure 2. Full-band effective-exposure map of the $7 \mathrm{Ms}$ CDF-S. The linear gray scale bar is shown in the upper right; the displayed effective exposure times are in units of Ms. The maximum effective exposure time is $6.651 \mathrm{Ms}$, which is smaller than the total cleaned exposure of $6.727 \mathrm{Ms}$ as the aim points of individual observations differ. The radial trails and central ring-like structure with relatively low effective exposures are caused by the ACIS-I CCD gaps. The regions and the plus sign are the same as those in Figure 1.

was assumed when creating the exposure maps, which is approximately the slope of the cosmic X-ray background spectrum in the full band (e.g., Marshall et al. 1980; Gendreau et al. 1995; Hasinger et al. 1998). The full-band effectiveexposure map is shown in Figure 2, with a maximum effective exposure time of $6.651 \mathrm{Ms}$.

From the effective-exposure maps, we can derive the survey solid angle as a function of the minimum effective exposure. For the purpose of comparing to the previous $2 \mathrm{Ms}$ and $4 \mathrm{Ms}$ CDF-S results (the definition of the full band and hard band have changed), we show in Figure 3 such a relation in the soft band. Approximately $49 \%$ of the $7 \mathrm{Ms}$ CDF-S field has $>3.5$ Ms effective exposure, while $3.5 \mathrm{Ms}$ is close to the deepest effective exposure achieved in the $4 \mathrm{Ms}$ CDF-S with only $3 \%$ of the field having longer exposure times. In the $7 \mathrm{Ms}$ CDF-S, $\approx 45 \%, 39 \%$, and $9.4 \%$ of the field have $>4 \mathrm{Ms},>5 \mathrm{Ms}$, and $>6 \mathrm{Ms}$ effective exposure, respectively. The survey solid-angle curves for the 4 Ms CDF-S (Xue et al. 2011) and 2 Ms CDF-S (Luo et al. 2008) are similar to the 7 Ms curve in the sense that they are approximately the scaled-down versions of the $7 \mathrm{Ms}$ curve with scaling factors of 1.8 and 3.5 in effective exposure, respectively. The overall larger solid angles of the $7 \mathrm{Ms} C D F-S$ compared to the $4 \mathrm{Ms}$ CDF-S suggest that we are not only able to detect new sources below the $4 \mathrm{Ms}$ CDF-S sensitivity limit, but also detect new sources above that limit owing to the significantly increased solid-angle coverage at any given exposure time.

We created exposure-corrected smoothed images in the 0.5-2.0 keV, 2-4 keV, and 4-7 keV bands following Section 3.3 of Baganoff et al. (2003) using the CIAO tool CSMOOTH. The images and effective-exposure maps were adaptively smoothed with the same scale map in each band, and the smoothed images were divided by the corresponding smoothed exposure maps. These exposure-corrected smoothed images

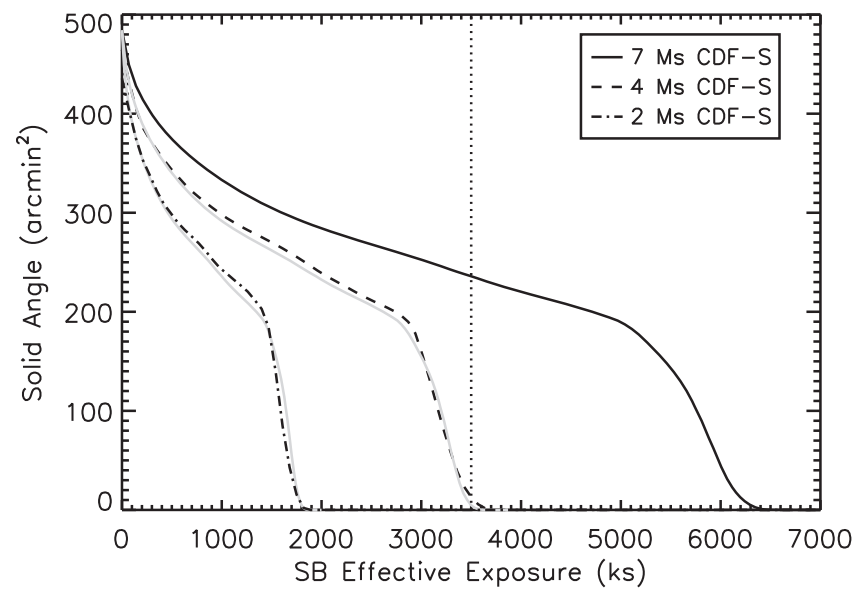

Figure 3. Survey solid angle as a function of minimum soft-band effective exposure for the $7 \mathrm{Ms}$ CDF-S (black solid curve). The vertical dotted line indicates an effective exposure of $3.5 \mathrm{Ms}$, and $235.9 \operatorname{arcmin}^{2}(49 \%)$ of the $7 \mathrm{Ms}$ CDF-S and $13.0 \operatorname{arcmin}^{2}(3 \%)$ of the $4 \mathrm{Ms}$ CDF-S have $>3.5 \mathrm{Ms}$ effective exposure. For comparison, the dashed and dash-dotted curves display the $4 \mathrm{Ms}$ CDF-S (Xue et al. 2011) and 2 Ms CDF-S (Luo et al. 2008) solid angles, which were derived following the same procedures as in this paper. The $7 \mathrm{Ms}$ curve can be roughly rescaled to $4 \mathrm{Ms}$ and 2 Ms curves with scaling factors of 1.8 and 3.5 in effective exposure (gray solid curves), respectively.

were combined to produce a color composite, as shown in Figure 4; an expanded view of the central $8^{\prime} \times 8^{\prime}$ region is also shown to illustrate the large abundance of sources near the field center. Although many of the X-ray sources are clearly visible in the adaptively smoothed images, our source searching was performed on the raw images (e.g., Figure 1), as detailed in Section 3.2 below. Our CSMOOTH processes were not optimized to enhance the visibility of extended sources (which requires external background files for proper computation of the source significance), and extended faint color halos which appear in Figure 4(b) are usually artifacts rather than real extended sources (e.g., Finoguenov et al. 2015).

\subsection{X-Ray Source Detection}

X-ray source detection for the $7 \mathrm{Ms}$ CDF-S follows the same two-stage approach as was employed in the Xue et al. (2011) $4 \mathrm{Ms}$ CDF-S catalog, which maximizes the number of reliable sources detected (e.g., Lehmer et al. 2009; Xue et al. 2011). A candidate source list was initially generated by WAVDETECT source detection, and it was then filtered by AE to produce our main source catalog, which includes significant X-ray sources that are unlikely to be false detections caused by background fluctuations. A supplementary source catalog was also produced that contains lower-significance X-ray sources with bright $\left(K_{s} \leqslant 23\right)$ NIR counterparts.

To generate the candidate source list, we ran WAVDETECT on the combined raw images in the full band, soft band, and hard band, using a " $\sqrt{2}$ sequence" of wavelet scales (i.e., 1, 1.414, 2 , $2.828,4,5.656,8,11.314$, and 16 pixels) and a false-positive probability threshold of $10^{-5}$. The WAVDETECT process made use of a merged PSF map, created by choosing the minimum PSF size at each pixel location among all the PSF maps of individual observations (e.g., see Section 2.2.2 of Xue et al. 2016); such a process is optimized for point-source detection. ${ }^{34}$ We merged the three source lists for the three bands into the candidate source list by cross matching them

34 See http://cxc.harvard.edu/ciao/threads/wavdetect_merged/index.html\#min. 

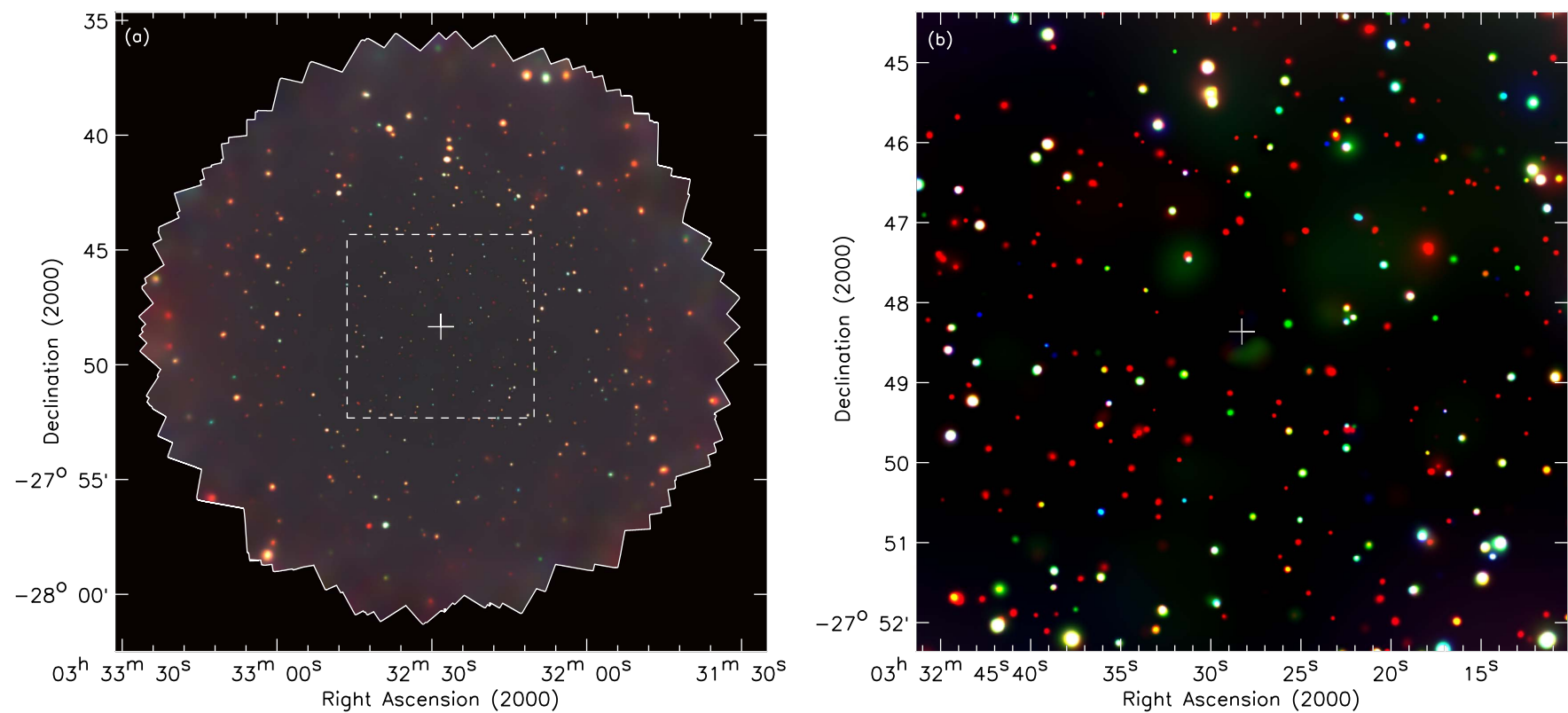

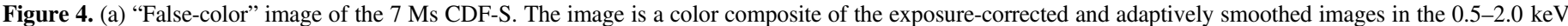

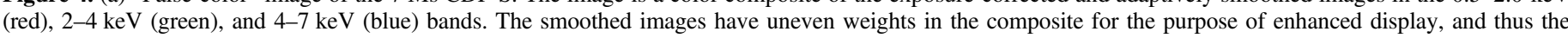

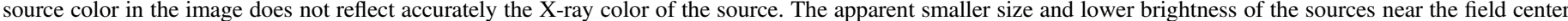

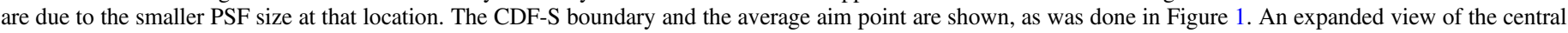
$8^{\prime} \times 8^{\prime}$ region (dashed square region) is displayed in panel (b). Extended faint color halos in panel (b) are usually artifacts instead of real extended sources.

with a matching radius of 2 " 5 for sources within $6^{\prime}$ of the average aim point and 4." 0 for sources at larger off-axis angles (the distance between the source position and the average aim point); we also visually inspected all of the sources beyond $8^{\prime}$ of the average aim point and removed nine candidate sources that are likely the same as their companion detections $\approx 4-7^{\prime \prime}$ away. The X-ray source positions in the merged source list were adopted from, in order of priority, the full-band, softband, and hard-band positions. The resulting candidate source list includes 1121 sources.

The relatively loose WAVDETECT source-detection threshold of $10^{-5}$ introduces a non-negligible number of spurious detections. We also performed WAVDETECT source searching with the more stringent false-positive probability thresholds of $10^{-6}, 10^{-7}$, and $10^{-8}$. We then assigned a minimum WAVDETECT false-positive probability to each of the 1121 candidate sources according to the minimum WAVDETECT threshold value at which the source was detected. Of the 1121 sources, 644, 58, 102, and 317 have minimum WAVDETECT false-positive probabilities of $10^{-8}, 10^{-7}, 10^{-6}$, and $10^{-5}$, respectively. Candidate sources with smaller minimum WAVDETECT false-positive probabilities are more likely real detections and most of the spurious detections will have minimum falsepositive probabilities of $10^{-5}$ (e.g., see Figure 5 below).

Before filtering the candidate source list with $\mathrm{AE}$, we improved the source positions through the AE "CHECK_POSITIONS" procedure. As was done in our previous CDF-S, Extended Chandra Deep Field-South (E-CDF-S), and Chandra Deep Field-North (CDF-N) catalogs (Luo et al. 2008; Xue et al. 2011, 2016), we adopted AE centroid positions for sources within $8^{\prime}$ of the average aim point and matched-filter positions for sources located at larger off-axis angles. ${ }^{35} \mathrm{We}$

\footnotetext{
35 The matched-filter position is the position found by correlating the merged image in the vicinity of a source with the combined source PSF (see Section 5.3 of the AE User's Guide).
}
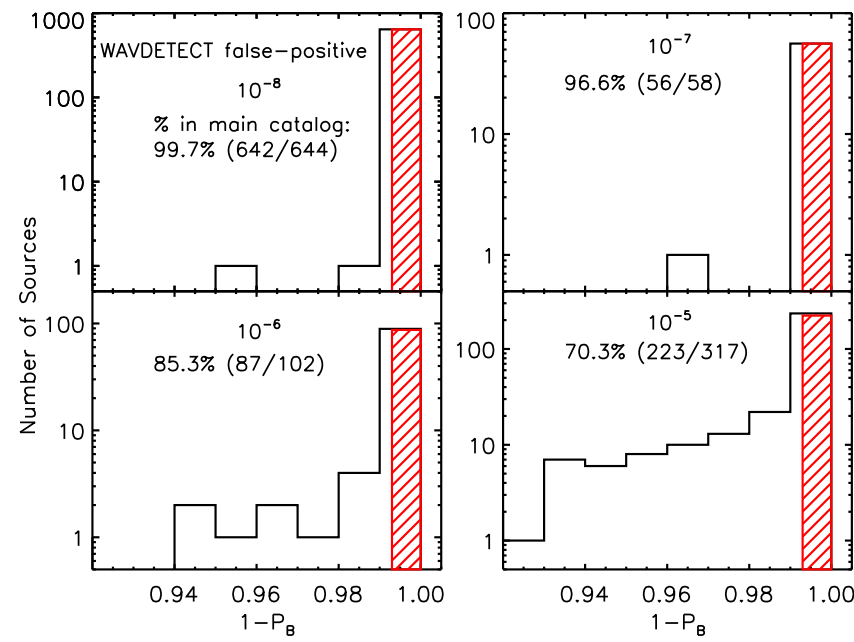

Figure 5. Distribution of 1 minus the AE binomial no-source probability $\left(P_{\mathrm{B}}\right)$ for sources in the candidate-list catalog with different minimum WAVDETECT false-positive probabilities. Sources having $P_{\mathrm{B}}<0.007$ were included in the main source catalog, and they are indicated by the red shaded bars, which have a slightly smaller width than the rightmost $P_{\mathrm{B}}$ bin $(0.007$ vs. 0.01$)$. The fraction of main-catalog sources among each minimum WAVDETECT false-positive bin is annotated, with the numbers of sources shown in the parentheses. The fraction drops from $99.7 \%$ at a minimum WAVDETECT false-positive probability of $10^{-8}$ to $70.3 \%$ at a minimum WAVDETECT false-positive probability of $10^{-5}$

further visually inspected the raw and adaptively smoothed images for each source and manually chose centroid or matched-filter positions for $\approx 60$ sources, which align better with the apparent source centers (mostly sources located within $6-8^{\prime}$ of the average aim point where the matched-filter positions are preferred).

We then utilized AE to extract photometric properties of the candidate sources. The details of the AE photometric extraction are described in the AE User's Guide; a short summary is 
presented in Section 3.2 of Xue et al. (2011). Briefly, AE performed source and background extractions for each source in each observation and then merged the results. A polygonal extraction region that approximates the $\approx 90 \% \mathrm{ECF}$ contour of the local PSF was utilized to extract source counts; smaller extraction regions $(\approx 40 \%-75 \%$ ECFs $)$ were used in crowded areas where sources have overlapping $\approx 90 \%$ ECF apertures. The AE "BETTER_BACKGROUNDS" algorithm was adopted for background extraction (Section 7.6.1 of the AE User's Guide), which seeks to obtain a single background region plus a background scaling that simultaneously models all background components, including the background that arises from the PSF wings of neighboring sources. A minimum number of 100 counts in the merged background spectrum is required to ensure photometric accuracy, which was achieved through the AE "ADJUST_BACKSCAL" stage. The extraction results from individual observations were then merged to produce photometry for each source through the AE "MERGE_OBSERVATIONS" procedure.

One important output parameter from AE is the binomial nosource probability, $P_{\mathrm{B}}$, which is the probability of still observing the same number of source counts or more under the assumption that there is no real source at the relevant location and the observed excess number of counts over background is purely due to background fluctuations. The formula to obtain $P_{\mathrm{B}}$ is given by

$$
P_{\mathrm{B}}(X \geqslant S)=\sum_{X=S}^{N} \frac{N !}{X !(N-X) !} p^{X}(1-p)^{N-X} .
$$

In this equation, $S$ is the total number of counts in the sourceextraction region and $B$ is the total number of counts in the background extraction region; $N$ is the sum of $S$ and $B$; $p=1 /(1+B A C K S C A L)$, with $B A C K S C A L$ being the area ratio of the background and source-extraction regions. A smaller $P_{\mathrm{B}}$ value indicates that a source has a larger probability of being real. For each source, AE computed a $P_{\mathrm{B}}$ value in each of the three bands, and we adopted the minimum of the three as the final $P_{\mathrm{B}}$ value for the source.

Although $P_{\mathrm{B}}$ is a classic confidence level, it is usually not a good indicator of the fraction of spurious sources (e.g., a cut at $P_{\mathrm{B}}=0.01$ does not correspond to a $1 \%$ spurious rate), mainly because the extractions were performed on a biased sample of candidate sources that already survived a filtering process by WAVDETECT. Furthermore, given its definition, the value of $P_{\mathrm{B}}$ also depends on the choices of source and background extraction regions. Therefore, we cannot reject spurious sources simply based on the absolute value of $P_{\mathrm{B}}$ itself. Fortunately, from past experience (e.g., Luo et al. 2010; Xue et al. 2011), we found that the superb multiwavelength coverage in the CDF-S allows us to identify counterparts for the majority $(\approx 96 \%)$ of the X-ray sources, and the accurate X-ray and optical/NIR/ radio source positions also ensure high-confidence associations (false-match rate $\approx 2 \%$ ). These combined factors indicate that $\mathrm{X}$-ray sources having a multiwavelength counterpart (down to the magnitude limits of the multiwavelength catalogs) are extremely likely to be real detections. Thus, we proceeded to choose a $P_{\mathrm{B}}$ threshold that retains a large number of sources with multiwavelength counterparts while removing most of the sources without counterparts. The multiwavelength catalogs used for identification and the identification procedure are the same as those described in Section 4.2 below. After evaluation of the matching results at several possible $P_{\mathrm{B}}$ threshold values (0.001-0.01), we adopted $P_{\mathrm{B}}<0.007$ as the criterion to prune the candidate source list and generate a main source catalog, which includes 1008 sources with a $\approx 97 \%$ multiwavelengthidentification rate. The detailed properties of the main-catalog sources are presented in Section 4 below.

The choice of the $P_{\mathrm{B}}$ threshold is an empirical decision, optimized to balance the needs of recovering a large number of real sources (high completeness), while keeping the fraction of potential spurious sources small (high reliability). A slightly different choice of the threshold value will affect the final source catalog, as well as the catalog completeness and reliability slightly; in fact, most of the main-catalog sources, 922 out of the $1008(91.5 \%)$, have $P_{\mathrm{B}}<0.001$ and are highly reliable ( $>98 \%$ identification rate). The $P_{\mathrm{B}}$ threshold value was 0.004 for the 2 Ms CDF-N (Xue et al. 2016) and 4 Ms CDF$\mathrm{S}$ (Xue et al. 2011), and it was 0.002 for the $250 \mathrm{ks}$ E-CDF-S (Xue et al. 2016). As reasoned above, the absolute $P_{\mathrm{B}}$ values are not directly comparable, but these choices were also made based on the multiwavelength identification results to optimize the balance between completeness and reliability, consistent with our current approach.

Our adopted $P_{\mathrm{B}}$ threshold will have inevitably rejected real $\mathrm{X}$-ray sources. To recover some of these real sources, we created a supplementary source catalog that contains lowersignificance X-ray sources that have bright optical/NIR counterparts, as has also been done for the 4 Ms CDF-S (Xue et al. 2011); the chance of a bright optical/NIR source being associated with a spurious X-ray detection is quite small. A total of 47 candidate CDF-S sources having $0.007 \leqslant P_{\mathrm{B}}<0.1$ are associated with bright, $K_{s} \leqslant 23$, TENIS sources, where the false-match rate is only $1.7 \%$, and these 47 sources constitute the supplementary catalog. The basic X-ray and multiwavelength properties of the supplementary catalog sources are presented in Section 5 below.

The distributions of $P_{\mathrm{B}}$ for sources in the candidate-list catalog with different minimum WAVDETECT false-positive probabilities are displayed in Figure 5. Sources that are detected by WAVDETECT at smaller false-positive probability thresholds are also considered more significant by $\mathrm{AE}$ with smaller $P_{\mathrm{B}}$ values in general. Most $(99.7 \%)$ of the candidate sources detected with minimum WAVDETECT false-positive probabilities of $10^{-8}$ remain in the main catalog, while a substantial fraction $(\approx 30 \%)$ of the candidate sources with minimum WAVDETECT false-positive probabilities of $10^{-5}$ were rejected by the $\mathrm{AE}$ filtering, likely being spurious detections. Of the 47 supplementary catalog sources, 1, 9, and 37 have minimum WAVDETECT false-positive probabilities of $10^{-8}, 10^{-6}$, and $10^{-5}$, respectively.

\section{MAIN CHANDRA SOURCE CATALOG}

After determining the main and supplementary catalog sources, we performed another AE photometric-extraction procedure on these catalog sources instead of the candidate sources; the exclusion of those rejected candidate sources affected the photometry of several sources slightly, due to changes in the extraction regions and/or background levels. The characterization of the X-ray and multiwavelength source properties, including X-ray positional uncertainties, multiwavelength counterparts, redshifts, X-ray photometric and basic spectroscopic properties, and AGN classification, follows similar approaches as those used in the Xue et al. (2011) 4 Ms 

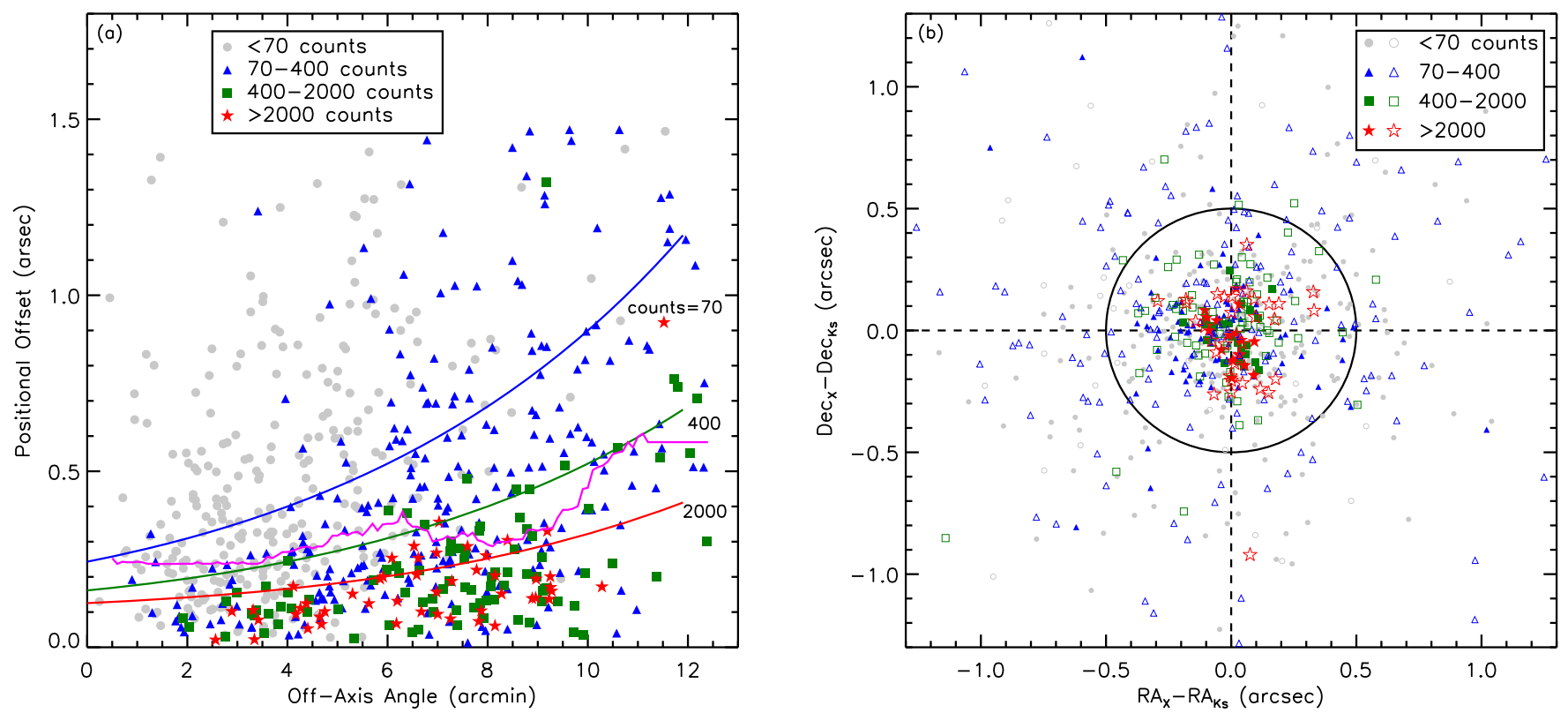

Figure 6. (a) Positional offset vs. off-axis angle for the 662 main-catalog sources that have a bright $\left(K_{s} \leqslant 22\right)$ TENIS counterpart with a matching radius of 1 "! 5 . The gray circles, blue triangles, green squares, and red stars represent X-ray sources with $<70,70-400,400-2000$, and $>2000$ counts, respectively. The magenta solid curve displays the running median of the positional offsets for all these sources in off-axis angle bins of $3^{\prime}$. These data were used to derive the $1 \sigma$ positional uncertainties of the X-ray sources (see Equation (2)). The blue, green, and red solid curves represent the quadratic sum of the $1 \sigma$ positional errors $\left(\sqrt{\sigma_{\mathrm{X}}^{2}+\sigma_{K s}^{2}}\right.$, where $\sigma_{K s}=0.1$ ) for sources with 70, 400, and 2000 counts, respectively; there are still broad ranges of counts for sources marked as blue triangles (70-400) and green squares (400-2000), and thus the blue and green curves are lower limits on the expected $1 \sigma$ positional offsets. Approximately, $68 \%(1 \sigma)$ of the blue triangles, green squares, and red stars lie below their corresponding solid curves, respectively. (b) Distribution of the positional offsets in the R.A. (RA) vs. decl. (Dec) plane for the 662 main-catalog sources having a bright TENIS counterpart. The symbols have the same meaning as in panel (a); additionally, filled and open symbols represent sources having an off-axis angle of $\leqslant 6^{\prime}$ and $>6^{\prime}$, respectively. The majority of the sources, especially those that are on axis and have a large number of counts, lie within the black circle, which has a radius of 0 ." 5 . For each of the four groups of sources in different count bins, the mean positional offsets in R.A. and decl. are consistent with zero within the uncertainties.

CDF-S catalog, and these are described in detail in the following subsections. A summary of the main-catalog data columns can be found in Section 4.8 below.

\subsection{X-Ray Source Positional Uncertainty}

We investigated the accuracy of the X-ray source positions by comparing them to the positions of the 6651 bright $\left(K_{s} \leqslant 22\right)$ TENIS sources that were used in Section 2.2 to register the astrometry of the CDF-S observations. We matched the X-ray sources to the $K_{s}$-band sources using a matching radius of 1."5; we removed manually two matches where the TENIS positions are significantly affected by source blending. There are 662 matches found, with a median positional offset of 0 !" 30 . The expected number of false matches is small, $\approx 27$ $(\approx 4.1 \%)$ estimated by shifting the X-ray source positions manually and recorrelating them to the TENIS $K_{s}$-band sources (e.g., see Section 3.3.1 of Luo et al. 2008); such a small falsematch rate does not affect our analysis of the X-ray source positional uncertainties below. The 1".5 matching radius was used here only to obtain X-ray source positional uncertainties; later, we adopt a more sophisticated likelihood-ratio matching technique, which takes the derived X-ray source positional uncertainties as input parameters, to identify multiwavelength counterparts for the X-ray sources (Section 4.2).

The positional offsets between the X-ray and $K_{s}$-band sources have clear off-axis angle and source-count dependencies, as illustrated in Figure 6(a). The former is caused by the broader Chandra PSF sizes at larger off-axis angles, and the latter is due to the fact that locating the centroid of a faint X-ray source is difficult. As was done in our previous CDF-S and
E-CDF-S catalogs (Luo et al. 2008; Xue et al. 2011, 2016), we derived an empirical relation for the $\mathrm{X}$-ray positional uncertainty adopting the basic functional form proposed by Kim et al. (2007a):

$$
\log \sigma_{\mathrm{X}}=0.0606 \theta-0.320 \log C-0.064
$$

In the above equation, $\sigma_{\mathrm{X}}$ is the $1 \sigma$ positional uncertainty in units of arcseconds, $\theta$ is the off-axis angle in arcminutes, and $C$ is number of source counts in the energy band where the source position was determined (Section 3.2); an upper limit of 2000 was set on $C$, as the positional accuracy does not improve significantly with larger numbers of counts. The coefficients of Equation (2) were determined so that for a given sample of $\mathrm{X}$-ray- $K_{s}$ matches, the fraction of sources having positional offsets smaller than expectations $\left(\sqrt{\sigma_{\mathrm{X}}^{2}+\sigma_{K s}^{2}}\right.$, where $\sigma_{K_{s}}=0$ ". 1 is the adopted TENIS source positional uncertainty) is $\approx 68 \% ; ;^{36}$ a few such examples are displayed in Figure 6(a). In Figure 6(b), we display the positional offsets in R.A. and decl. Most of the X-ray sources with large numbers of counts (e.g., $>400$ ) or small off-axis angles (e.g., $<6^{\prime}$ ) have their positions determined reliably to within $\approx 0$ " 5 . Considering the entire X-ray source sample, there is no systematic offset in R.A. or decl. when compared to the $K_{s}$-band sources, as required by our astrometric registration (Section 2.2). For the main-catalog sources, the positional uncertainties range from 0 !" 11 to 1 !" 28 ,

\footnotetext{
36 Based on similar practices, the $90 \%$ and $95 \%$ confidence-level positional uncertainties are approximately 1.6 and 2.0 times the $1 \sigma$ positional uncertainties.
} 


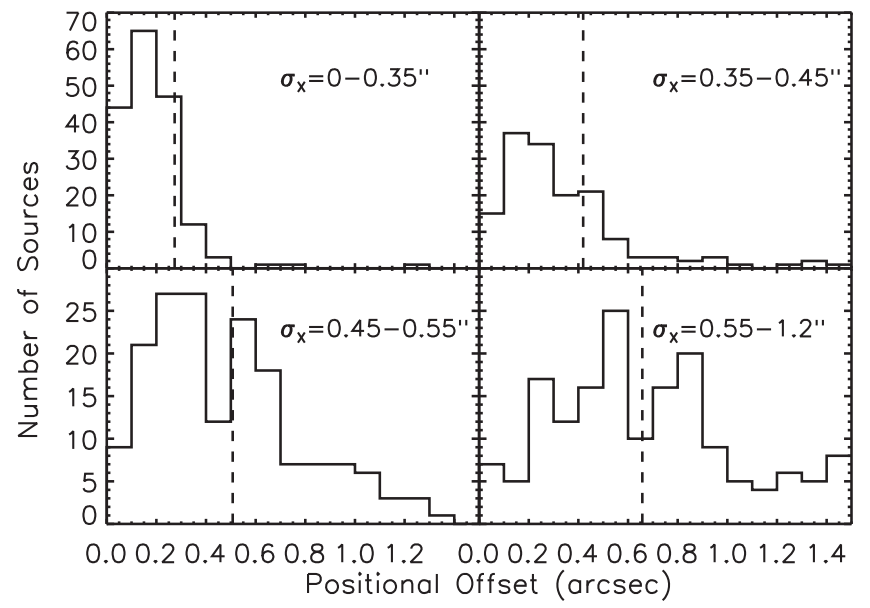

Figure 7. Histograms of the distributions of positional offsets for the 662 maincatalog sources that have a bright TENIS counterpart. These sources were divided into four bins according to their positional uncertainties, and each bin contains approximately the same number of sources. The vertical dashed line in each panel indicates the expected positional offset for each group of sources, which is the quadratic sum of the median X-ray positional uncertainty and the TENIS source positional uncertainty $\left(\sqrt{\sigma_{\mathrm{X} \text {,median }}^{2}+\sigma_{K s}^{2}}\right.$, where $\sigma_{K s}=0$.! 1 ), and $\approx 68 \%(1 \sigma)$ of the sources have a positional offset smaller than this value.

with a median value of 0.47 . Figure 7 presents the distributions of X-ray- $K_{s}$ positional offsets in four bins of $\mathrm{X}$-ray positional uncertainties; the offsets are consistent with expectations from the positional uncertainties.

\subsection{Multiwavelength Source Identifications}

We searched for optical, NIR, infrared (IR), and radio counterparts for the X-ray sources, following the likelihoodratio matching technique described in Luo et al. (2010). This technique computes a likelihood ratio for each potential counterpart, taking into account the positional uncertainties of the X-ray and optical/NIR/IR/radio sources as well as the expected magnitude distribution of counterparts. A threshold value for the likelihood ratio that maximizes the matching completeness and reliability was chosen to determine the final matches (see Section 2.2 of Luo et al. 2010 for details); in cases where multiple counterpart candidates satisfy the threshold cut for a single $\mathrm{X}$-ray source $(\approx 3 \%$ of the total main-catalog sources on average), we selected the candidate with the highest likelihood ratio. The false-match probability is estimated based on the Broos et al. (2011) shift-and-recorrelate Monte Carlo method, and our approach is described in detail in Section 4.3 of Xue et al. (2011). This approach does not account for any potential false matches introduced when only the highest likelihood-ratio counterpart was selected in the cases of multiple candidates. For example, if the observed X-ray emission comes from a high-redshift obscured AGN and it is gravitationally lensed by a foreground AGN/galaxy, the foreground object could potentially have a higher matching likelihood ratio and would be falsely selected as the counterpart. Lensing has affected the identification of submillimeter galaxies (SMGs), and $\approx 5 \%$ of bright SMGs are lensed (e.g., Blain 1996; Chapman et al. 2002; Simpson et al. 2015; Danielson et al. 2016). Our X-ray sources are located at a smaller median redshift ( $\approx 1.6$ for AGNs) than typical SMGs $(\approx 2.5)$, and the lensed fraction is likely much lower. We estimate that such potential false matches have a negligible contribution to the overall false-match rate derived from the likelihood-ratio matching approach.

Multiwavelength identifications were performed with the following seven optical-radio catalogs (in order of increasing wavelength), ${ }^{37}$ and a primary counterpart was chosen from one of these catalogs when available.

1. The Wide Field Imager (WFI) $R$-band catalog, with a $5 \sigma$ limiting magnitude of 27.3 (Giacconi et al. 2002; Giavalisco et al. 2004).

2. The GOODS-S HST version $\mathrm{r} 2.0 \mathrm{z}$ z-band catalog, with a $5 \sigma$ limiting magnitude of 28.2 (Giavalisco et al. 2004). ${ }^{38}$ This catalog covers a solid angle of $\approx 160 \operatorname{arcmin}^{2}$ in the center of the CDF-S (Figure 1).

3. The Galaxy Evolution from Morphologies and SEDs (GEMS) HST $z$-band catalog, with a $5 \sigma$ limiting magnitude of 27.3 (Caldwell et al. 2008). The GEMS survey complements the GOODS-S survey and covers the entire remaining area of the CDF-S that is not covered by GOODS-S.

4. The CANDELS + 3D-HST HST WFC3 F125W+F140W +F160W combined catalog (hereafter the CANDELS catalog; Skelton et al. 2014). The magnitudes in the F125W band were used, which has a $5 \sigma$ limiting magnitude of 28.3 .

5. The TENIS $K_{s}$-band catalog, with a $5 \sigma$ limiting magnitude of 25.0 in the inner $400 \mathrm{arcmin}^{2}$ region (Hsieh et al. 2012).

6. The Spitzer Extended Deep Survey (SEDS) Infrared Array Camera (IRAC) $3.6 \mu \mathrm{m}$ catalog, with a $3 \sigma$ limiting magnitude of $\approx 26$ (Ashby et al. 2013).

7. The Very Large Array (VLA) $1.4 \mathrm{GHz}$ catalog from Miller et al. (2013), with a $5 \sigma$ limiting flux density of $\approx 40 \mu \mathrm{Jy}$.

The absolute astrometry for each of the above catalogs was registered to the TENIS astrometric frame before the matching; the systematic offsets were negligible except for the VLA catalog, where $\approx 0$ "! 2 shifts in R.A. and decl. were required (see Footnote 30). Compared to the multiwavelength catalogs used for source identification in the $4 \mathrm{Ms}$ CDF-S catalog (Xue et al. 2011), additional deep NIR and IR survey catalogs have become available, including the TENIS, CANDELS, and SEDS catalogs, which aided greatly with the $7 \mathrm{Ms}$ source identification. For each X-ray source that has at least one match from these catalogs, we chose a primary counterpart from, in order of priority, the CANDELS, GOODS-S, GEMS, TENIS, VLA, WFI, and SEDS catalogs. This order was empirically determined based on the combined factors of positional accuracy, sensitivity, and potential source-blending problems (e.g., in the SEDS IRAC catalog). We identified primary counterparts for $982(97.4 \%)$ of the 1008 main-catalog sources, and $701,26,186,49,4$, and 16 of them are from the CANDELS, GOODS-S, GEMS, TENIS, WFI, and SEDS catalogs, respectively. There were no primary counterparts selected from the VLA catalog. The false-match probabilities for the matches found in the seven catalogs range from $0.14 \%$ (VLA) to $4.0 \%$ (WFI). For each of 982 matched X-ray sources, we consider its false-match probability to be the minimum one

\footnotetext{
${ }^{37}$ We also examined the GOODS-S MUlticolor Southern Infrared Catalog (MUSIC) v2 $K$-band catalog (Grazian et al. 2006) and the Multiwavelength Survey by Yale-Chile (MUSYC) $K$-band catalog (Taylor et al. 2009). These two catalogs do not provide any additional useful counterpart information, and thus we do not list them here.

${ }^{38}$ See http://archive.stsci.edu/pub/hlsp/goods/catalog_r2/.
} 


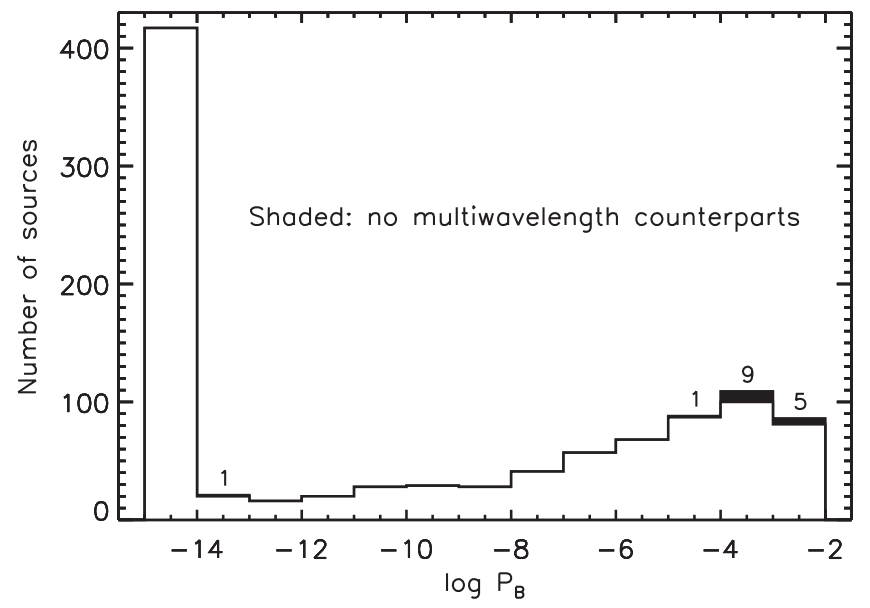

Figure 8. Distribution of the AE binomial no-source probability $\left(P_{\mathrm{B}}\right)$ for the main-catalog sources; sources with $\log P_{\mathrm{B}}<-15$ are plotted in the $\log P_{\mathrm{B}}=-15$ to $-14 \mathrm{bin}$. The shaded regions indicate sources that have no multiwavelength counterparts, with the numbers of such sources shown on the top of each bin. A significant fraction of the 16 sources that lack counterparts are likely spurious detections.

among the false-match probabilities of the optical through radio catalogs where the X-ray source has a match. For example, if the counterpart of an X-ray source is in the WFI catalog only, its false-match probability is $4.0 \%$; if the counterpart is in both the WFI and VLA catalogs, its false-match probability is $0.14 \%$; if the counterpart is in all seven catalogs, its false-match probability is $0.14 \%$. The mean false-match rate for the entire sample, derived by averaging the false-match probabilities of individual sources, is $1.6 \%$.

We examined the $26 \mathrm{X}$-ray sources that lack counterparts, and manually assigned multiwavelength matches to 10 of them. Six of these X-ray sources have a CANDELS companion $\approx 1$ ". $0-1$ ". 6 away, and their likelihood ratios fell slightly below our threshold value for matches. The other four X-ray sources are within the extent $\left(2\right.$ ! $^{\prime \prime}-4$. $\left.^{\prime \prime}\right)$ of low-redshift galaxies $(z=0.038-0.215)$ upon visual inspection, and they are probably off-nuclear sources associated with the galaxies (e.g., ultraluminous X-ray sources; ULXs) that are $\approx 4-12 \mathrm{kpc}$ away from the nuclei (e.g., Lehmer et al. 2006). These 10 manually matched sources are noted in Column 20 of the main-catalog table. In total, multiwavelength counterparts were identified for 992 (98.4\%) of the main-catalog sources. The false-match rate is around $1.6 \%$ or slightly higher, considering any possible additional false matches in the 10 manually matched cases, and it could be as large as $\approx 2.5 \%$ in the extremely unlikely case that all the 10 manual matches are incorrect. For the $16 \mathrm{X}$-ray sources without counterparts, we expect that a significant fraction or even most of them are spurious detections, as discussed in our AE source-filtering stage (Section 3.2). The locations of these 16 sources in the $P_{\mathrm{B}}$ distribution for the main-catalog sources are shown in Figure 8, and they indeed have large $P_{\mathrm{B}}$ values in general, indicating less-significant detections. We discuss the one source that is detected significantly $\left(\log P_{\mathrm{B}} \approx-13\right)$ but has no counterpart in Section 4.7.2 below.

There are three pairs of X-ray sources (XIDs 431, 432, 556, $558,649,653)$ that were matched to the same counterparts, which are galaxies at redshifts of $0.038,0.075$, and 0.579 , respectively. The pair sources further away from the galactic centers could be off-nuclear sources associated with the galaxies (e.g., Lehmer et al. 2006). For one pair of sources (XIDs 556 and 558), a strong radio counterpart is observed $(1.4 \mathrm{GHz}$ flux density $452 \mu \mathrm{Jy})$, and the two X-ray sources could be X-ray emission associated with extended radio jets/ lobes. These pair sources are noted in Column 20 of the maincatalog table, and we further noted another possible off-nuclear source (XID 761) in this column.

For the 992 main-catalog sources with primary counterparts, we further searched for their multiwavelength photometric properties by matching the primary counterparts to the other optical-radio catalogs above with a matching radius of 0 ". 75 or 1 ". 0 (for the VLA catalog only). The multiwavelength information for the 992 sources in the WFI, GOODS-S, GEMS, CANDELS, TENIS, SEDS, and VLA catalogs are presented in Columns 21-41 of the main-catalog table.

\subsection{Spectroscopic and Photometric Redshifts}

The superior multiwavelength data in the CDF-S allow collection of spectroscopic and photometric redshifts for the majority of the identified X-ray sources. We matched the primary counterparts to spectroscopic and photometric catalogs using a matching radius of 0.5 or 0.75 (if the primary counterpart is from the SEDS IRAC catalog). Spectroscopic redshifts were searched for in $\approx 30$ public catalogs, including a few X-ray spectroscopic redshift results, and redshifts for 665 of our main-catalog sources were collected from 26 of these catalogs, listed below. (1) Colless et al. (2003), (2) Szokoly et al. (2004), (3) Zheng et al. (2004), (4) Doherty et al. (2005), (5) Mignoli et al. (2005), (6) Ravikumar et al. (2007), (7) Kriek et al. (2008), (8) Vanzella et al. (2008), (9) Treister et al. (2009), (10) Wuyts et al. (2009), (11) Balestra et al. (2010), (12) Silverman et al. (2010), (13) Casey et al. (2011), (14) Feruglio et al. (2011), (15) Xia et al. (2011), (16) Cooper et al. (2012), (17) Iwasawa et al. (2012), (18) Mao et al. (2012), (19) Kurk et al. (2013), (20) Le Fèvre et al. (2013), (21) Rauch et al. (2013), (22) Del Moro et al. (2014), (23) Hsu et al. (2014), (24) Morris et al. (2015), (25) Santini et al. (2015), and (26) Tasca et al. (2016). The spectroscopic redshifts were flagged as "Secure" or "Insecure" depending on whether they were obtained from several reliable spectral features with $\gtrsim 95 \%$ confidence level. The spectroscopic redshifts for Galactic stars were set to zero; there are 12 stars in our catalog. The spectroscopic redshifts, quality flags, and the catalogs that the redshifts were collected from (numbered 1-26 as cited above) are presented in Columns 42-44 of the main-catalog table. We collected photometric redshifts from the following public catalogs: (1) Luo et al. (2010), (2) Rafferty et al. (2011), (3) Hsu et al. (2014), (4) Skelton et al. (2014), (5) Santini et al. (2015), and (6) Straatman et al. (2016). Unlike spectroscopic redshifts, photometric redshifts from different catalogs sometimes do not agree with each other, and thus we present all the available photometric redshifts in Columns 45-50 of the maincatalog table. In total, 985 of our main-catalog sources have at least one photometric redshift. ${ }^{39}$

For each source, we adopted a preferred redshift from, in order of priority, (1) a secure spectroscopic redshift, (2) an insecure spectroscopic redshift that agrees with at least

\footnotetext{
39 The counterpart of XID 679 is blended with a brighter optical/NIR source $\approx 1$ !' 6 away; the two sources are only resolved in the CANDELS catalogs among the optical through radio catalogs we examined. The spectroscopic and photometric redshifts collected for XID 679 are likely based on the spectroscopic and photometric properties of the companion object.
} 

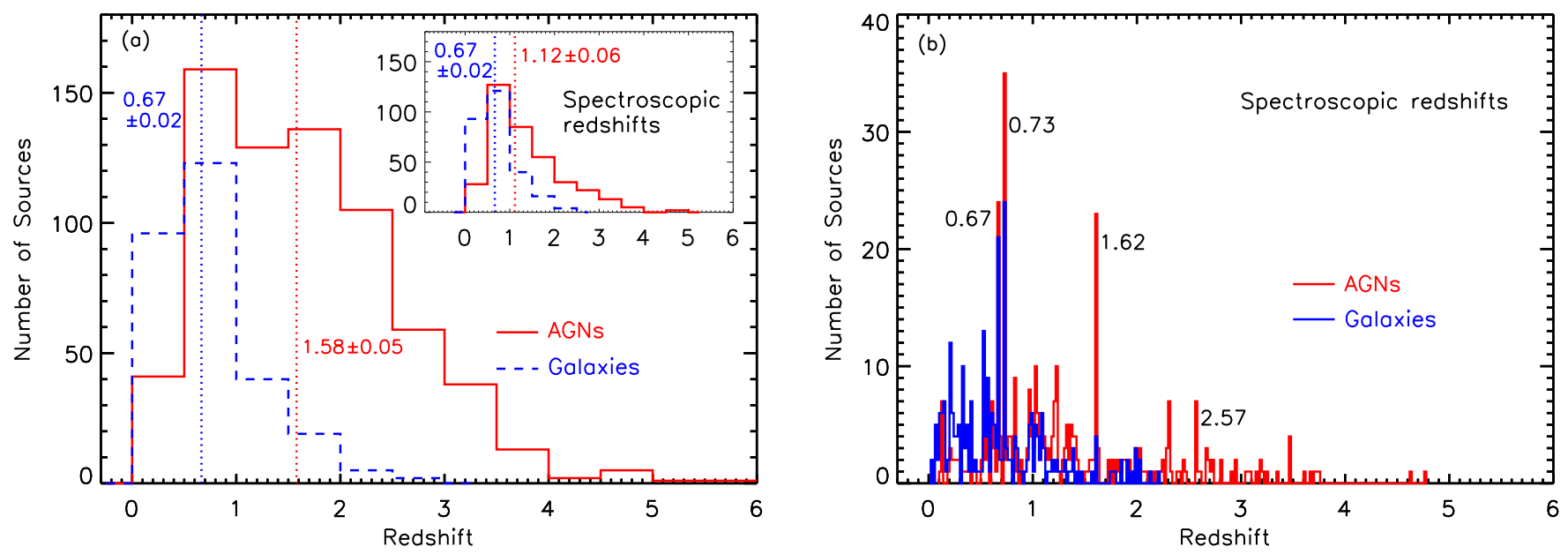

Figure 9. (a) Redshift distributions for the 689 AGNs (red histogram) and 285 galaxies (blue histogram) in bins of $\Delta z=0.5$. The inset displays the redshift distribution for the 367 AGNs and 274 galaxies with final adopted spectroscopic redshifts. The vertical dotted lines indicate the median redshift for every distribution, and the corresponding median values and their $1 \sigma$ uncertainties (derived via bootstrapping) are listed. (b) Redshift distributions for the 367 AGNs and 274 galaxies with final adopted spectroscopic redshifts in bins of $\Delta z=0.02$. Some of the prominent redshift spikes are noted.

one of its photometric redshifts to within $15 \%\left(\mid z_{\text {phot }}-\right.$ $z_{\text {spect }} /\left(1+z_{\text {spec }}\right) \leqslant 0.15 ;$ an empirical choice driven by experience), (3) a Hsu et al. (2014) photometric redshift, (4) a Luo et al. (2010) photometric redshift, (5) a Straatman et al. (2016) photometric redshift, (6) a Skelton et al. (2014) photometric redshift, (7) a Santini et al. (2015) photometric redshift, (8) a Rafferty et al. (2011) photometric redshift, and (9) an insecure spectroscopic redshift (when it is the only redshift available). The Hsu et al. (2014) photometric redshifts were preferred, in general, among all the photometric redshifts because of the combined factors that (1) Hsu et al. (2014) is a dedicated study of the CDF-S photometric redshifts, (2) it utilized intermediate-band photometric data, (3) the resulting photometric redshifts have good accuracy overall, (4) the details of the SED fitting of individual sources are publicly available, and (5) the highest fraction (94\%) of our main-catalog sources have matches in this catalog. In addition, for 16 sources, we adopted the Luo et al. (2010) photometric redshifts instead of the Hsu et al. (2014) photometric redshifts after reviewing the SED fitting plots.

Out of the 992 main-catalog sources with a primary counterpart, 986 (99.4\%) have final adopted redshifts, and we present these preferred redshifts and their origins in Columns 51-52 of the main-catalog table. For those adopted photometric redshifts, we also quote their $1 \sigma$ uncertainties in Columns 53-54 of the table, although we caution that these uncertainties often underestimate the real errors (e.g., see Section 3.4 of Luo et al. 2010). The redshift distributions for the main-catalog sources are shown in Figure 9(a). The median redshift for all the X-ray sources is $1.12 \pm 0.05$, with an interquartile range of $0.67-1.95$, where the $1 \sigma$ uncertainty on the median value was derived via bootstrapping. ${ }^{40}$ In Figure 9 (b), we display the distributions of spectroscopic redshifts in fine redshift bins $(\Delta z=0.02)$; there are some prominent redshift spikes indicative of X-ray large-scale structures (e.g., $z=0.67,0.74,1.62$, and 2.57; e.g., Gilli et al. 2003; Silverman et al. 2010; Dehghan \& Johnston-Hollitt 2014; Finoguenov et al. 2015). In the main catalog, there are two sources having $z>5$, and both are photometric redshifts. XID 172 is at

\footnotetext{
${ }^{40}$ See http://www.stat.wisc.edu/ larget/math496/bootstrap.html.
}

$z \approx 5.2$ from Hsu et al. (2014), and it has $z \approx 5.7$ from Luo et al. (2010) and $z \approx 7.7$ from Straatman et al. (2016); it is outside the CANDELS region and has TENIS, SEDS, and VLA counterparts. XID 238 is at $z \approx 5.8$ from Skelton et al. (2014); it only has CANDELS and VLA counterparts. ${ }^{41}$ XID 172 is likely X-ray absorbed ( $\left.\Gamma_{\text {eff }}=1.1\right)$, and XID 238 appears to be a soft X-ray source $\left(\Gamma_{\text {eff }}=2.3\right)$. It is probable that both sources are high-redshift AGNs.

Of the 986 final adopted redshifts, 653 are spectroscopic redshifts (including the 12 Galactic stars) and 333 are photometric redshifts. Most (284/333) of the photometric redshifts are from Hsu et al. (2014). We assess the quality of the Hsu et al. (2014) photometric redshifts by comparing them to the available secure spectroscopic redshifts for our sources. The comparison was performed for the 290 AGNs and 256 galaxies (see Section 4.5 below for AGN classification) in the main catalog which have both photometric redshifts from Hsu et al. (2014) and secure spectroscopic redshifts. We calculated the fraction of outliers defined as having $\mid z_{\text {phot }}-z_{\text {specl }} /\left(1+z_{\text {spec }}\right)>0.15$, and we estimated the accuracy of the photometric redshifts by computing the normalized median absolute deviation of the redshift differences, defined as $\sigma_{\mathrm{NMAD}}=1.48 \times$ median $\left(\left|z_{\text {phot }}-z_{\text {spec }}-\operatorname{median}\left(z_{\text {phot }}-z_{\text {spec }}\right)\right| /\left(1+z_{\text {spec }}\right)\right)$ (e.g., Luo et al. 2010). The results are presented in Figure 10. The photometric redshifts are of high quality, in general, especially for the galaxies. Note that some of the spectroscopic redshifts were used to train the SED templates in Hsu et al. (2014), which may bias the results toward better accuracy. The quality of the photometric redshifts also appears to depend on source brightness; Figure 11 shows that the outlier fraction for the AGN photometric redshifts increases toward larger $K_{s}$-band magnitudes (see also, e.g., Section 3.4 of Luo et al. 2010). Among the 333 sources with adopted photometric redshifts ( 322 AGNs), 93 have $K_{s} \leqslant 22(\approx 5 \%$ outlier fraction according to Figure 11$)$, 146 have $22<K_{s} \leqslant 24(\approx 13 \%$ outlier fraction), and 94 have $K_{s}>24$. There is not a sufficient number of spectroscopic redshifts at $K_{s}>24$ for assessing the quality of these photometric redshifts. Assuming arbitrarily a 15\% (30\%) outlier fraction for sources with $K_{s}>24$, the average outlier fraction for

\footnotetext{
41 The VLA counterpart is 1 !" 3 away from the CANDELS counterpart, farther than our adopted $1{ }^{\prime \prime} 0$ matching radius. We manually assigned the match.
} 

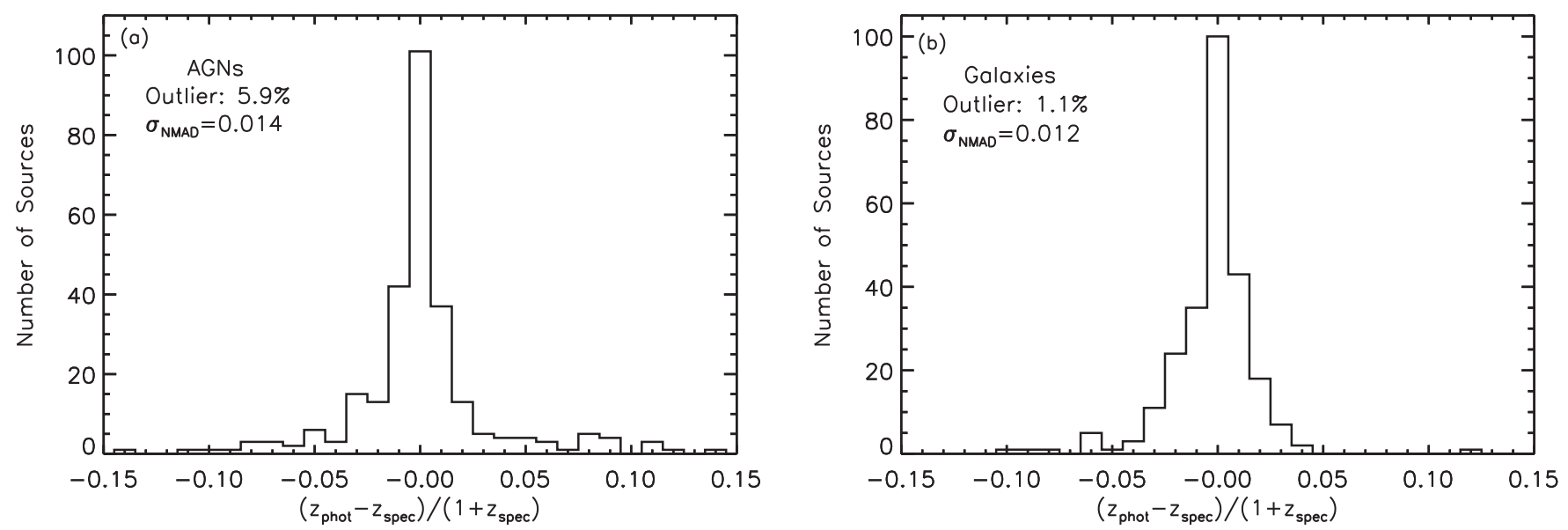

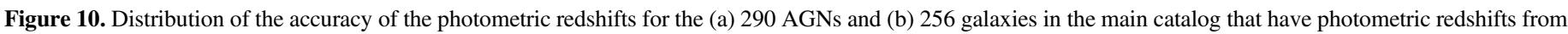
Hsu et al. (2014) and also secure spectroscopic redshifts. The outlier fractions and the redshift accuracy indicators $\left(\sigma_{\mathrm{NMAD}}\right)$ are displayed in each panel.

the 333 sources with adopted photometric redshifts is $\approx 11 \%(16 \%)$.

\subsection{X-Ray Photometric and Basic Spectroscopic Properties}

The aperture-corrected net source counts were derived from the AE extraction results. For each source in each of the three $\mathrm{X}$-ray bands, if the $P_{\mathrm{B}}$ value is less than our adopted threshold (0.007), we consider it as being detected and present in the main catalog the number of source counts along with the associated $1 \sigma$ statistical uncertainties computed by $\mathrm{AE}$ following Gehrels (1986), otherwise it is considered undetected and we present the $90 \%$ confidence-level upper limit on the source counts following the Kraft et al. (1991) Bayesian method. The aperture-correction factors were derived from the energy-dependent correction factors estimated by $\mathrm{AE}$ for individual observations (see Section 3.2 of Xue et al. 2011 for details), and the median correction factors for the full, soft, and hard bands are $0.885,0.907$, and 0.843 , respectively. The net source counts and their uncertainties are presented in Columns 8-16 of the main-catalog table. Sources near the edge of the CDF-S field have relatively low effective exposure times, large PSF sizes, non-uniform local background, and sometimes substantially varying (up to a factor of a few) effective exposure times within the extraction apertures. Additional photometric uncertainties for these sources might arise besides the statistical uncertainties presented in the current catalog, especially for faint sources. We noted 45 such sources in Column 17 of the main-catalog table (marked with "E") that are covered by less than 20 of the 102 CDF-S observations (4-19 observations); these sources all have large off-axis angles $(9 ! 9-12 ! 4)$ and relatively low effective exposure times (37-847 ks). We also noted in Column 17 another 34 sources that are in crowded regions (marked with "C") and were extracted using $\approx 40 \%-74 \%$ ECF apertures instead of the standard $\approx 90 \%$ ECF apertures; the photometry of these sources might still have some mild contamination from the companion sources.

The distributions of the source counts in the three bands are displayed in Figure 12. Table 2 summarizes the basic statistics of the source counts in the three bands; the median numbers of detected counts in the full, soft, and hard bands are $98.9 \pm 6.1$, $47.7 \pm 2.0$, and $94.6 \pm 6.0$, respectively, where the $1 \sigma$ uncertainties on the median values were derived via bootstrapping. In

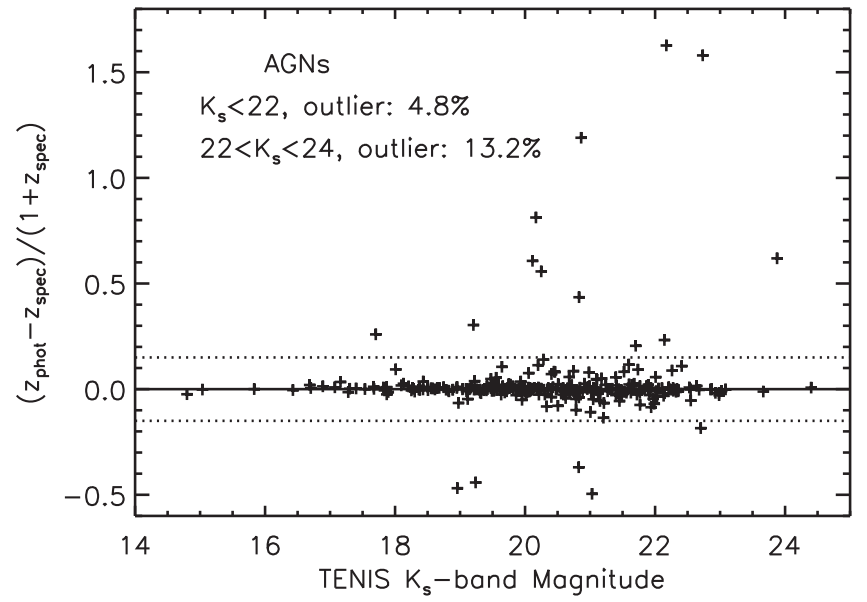

Figure 11. Photometric redshift accuracy vs. TENIS $K_{s}$-band magnitude for the AGNs in the main catalog that have photometric redshifts from Hsu et al. (2014) and also secure spectroscopic redshifts. The solid and dashed lines indicate $\left(z_{\text {phot }}-z_{\text {spec }}\right) /\left(1+z_{\text {spec }}\right)$ values of $0,0.15$, and -0.15 , respectively. The outlier fraction of the photometric redshifts increases toward larger $K_{s}$ band magnitudes.

Table 3, we provide the numbers of sources detected in one band but not another; there are 22 sources detected only in the full band, 84 only in the soft band, and 8 only in the hard band. There are 456 sources with $>100$ full-band counts, allowing basic spectral analyses, and there are 90 sources with $>1000$ full-band counts.

While detailed spectral analyses of the X-ray sources are beyond the scope of the current study and will be presented in additional papers (e.g., T. Liu et al. 2017, in preparation; Yang et al. 2016), we still derived basic spectroscopic properties for the catalog sources. Assuming the $0.5-7.0 \mathrm{keV}$ spectra of the $\mathrm{X}$-ray sources are power laws modified by only Galactic absorption, we derived the effective power-law photon indices $\left(\Gamma_{\text {eff }}\right)$ from the hard-to-soft band ratios. The band ratio is defined as the ratio between the hard-band and soft-band count rates, and the count rate was computed by dividing the aperture-corrected net counts by the effective exposure time. For the 502 sources detected in both the soft and hard bands, we computed the $1 \sigma$ uncertainties of the band ratios using the Bayesian code BEHR (Park et al. 2006). For the 479 sources detected in either the soft or hard band, but not both, we adopted the mode values of the band-ratio probability density 


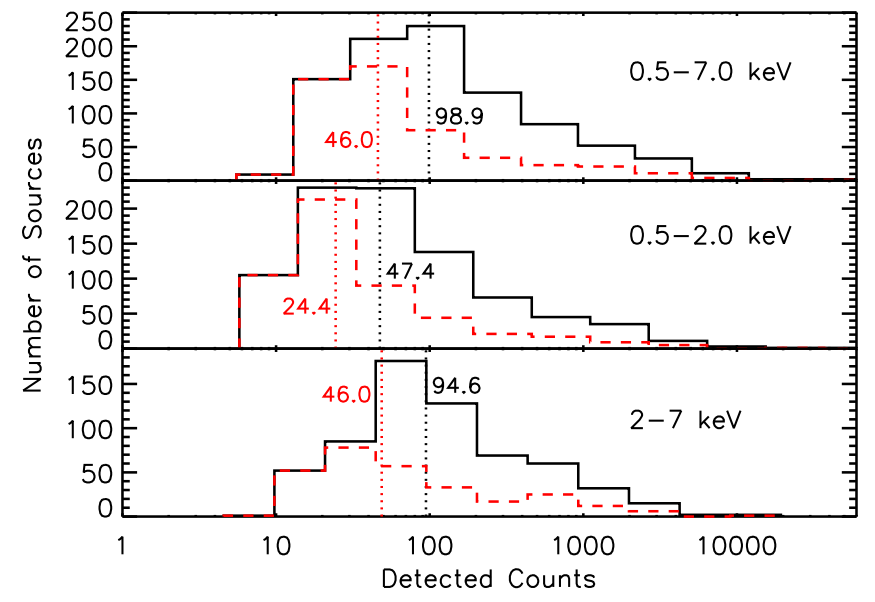

Figure 12. Distributions of source counts for the main-catalog sources in the full (top), soft (middle), and hard (bottom) bands. The black solid histograms show the count distributions for all the sources in the CDF-S field, while the red-dashed histograms show the distributions for the subgroups of sources within $6^{\prime}$ of the average aim point. Sources with upper limits on the counts are not included in the plots. The vertical dotted lines indicate the median numbers of counts for every distribution, and the corresponding median values are listed.

distributions calculated using BEHR as the band ratios; these are not upper or lower limits, but best-guess estimates. ${ }^{42}$ These band ratios were only used for estimating $\Gamma_{\text {eff }}$, source fluxes, and intrinsic absorption column densities, and their uncertainties were not calculated. For the 22 sources detected only in the full band, the band ratios cannot be constrained, and $\Gamma_{\text {eff }}=1.4$ was adopted for them. For each source, we calibrated the relation between the effective photon index and band ratio using simulated spectra produced by the FAKEIT command in XSPEC (version 12.9.0; Arnaud 1996) with the AE-generated merged spectral response files for this source. The band-ratioto- $\Gamma_{\text {eff }}$ conversion factors differ slightly for different sources. The uncertainties on $\Gamma_{\text {eff }}$ were calculated following the error propagation method in Section 1.7.3 of Lyons (1991). The band ratios and effective photon indices are presented in Columns 58-63 of the main-catalog table.

Figure 13 illustrates the band ratio as a function of full-band count rate for the main-catalog sources; the corresponding average $\Gamma_{\text {eff }}$ and full-band flux values are also shown. We split the sources into several count-rate bins and present their stacked count rates and band ratios, and we investigated these average values for the AGN and galaxy populations respectively (see Section 4.5 below for source classification). For the AGNs, the average band ratio rises when the count rate declines from $\approx 10^{-2}$ to $\approx 10^{-4}$ counts $\mathrm{s}^{-1}$, and it drops as the count rate decreases further below $\approx 10^{-5}$ counts $\mathrm{s}^{-1}$. A similar trend is present if we compute the average band ratios using the median values instead of stacking. The rise in band ratio (decrease in $\Gamma_{\text {eff }}$ ) toward lower fluxes (harder when fainter) at high count rates has been observed in previous deep surveys (e.g., Alexander et al. 2003; Lehmer et al. 2005; Luo et al. 2008; Xue et al. 2011), and it is caused by an increase in the fraction of absorbed AGNs detected at lower fluxes. The decline at the lowest count rates (softer when fainter) was also weakly present in the $4 \mathrm{Ms}$ CDF-S (Figure 18 of Xue et al. 2011), and it is likely due to the bias of preferentially

\footnotetext{
42 Although considerably uncertain, these values appear more appropriate for computing the source fluxes than assuming simply a uniform spectral shape (e.g., $\Gamma_{\text {eff }}=1.4$ ) for all such sources.
}

detecting soft-band sources at the lowest flux levels. At high count rates (high fluxes), the numbers of soft-band and hardband detected sources are comparable, while at a count rate of $10^{-5}$ counts $\mathrm{s}^{-1}$, the CDF-S area that is sensitive for detecting such a soft-band source is $\approx 1.5$ times the area that is sensitive for detecting a hard-band source (see Section 7 below for the sensitivity analysis), and a similar ratio is observed between the detected numbers of soft-band and hard-band sources. The sensitivity difference is more pronounced at even lower count rates, and thus it could cause the apparent softer-when-fainter trend. If we stacked only sources that are detected in both the soft and hard bands, or if we stacked only sources within the innermost $3^{\prime}$-radius region where the soft-band and hard-band sensitivity difference is small, the average band ratio does not appear to drop below the count rate of $10^{-5}$ counts $\mathrm{s}^{-1}$. This bias should also be responsible, at least partially, for a similar trend observed for galaxies in the low count-rate regime in Figure 13.

Using XSPEC and the AE-generated merged spectral response files for each source, we converted the count rate or upper limit on the count rate to the corresponding flux or flux upper limit, assuming that the spectrum is a power law having a photon index of $\Gamma_{\text {eff }}$ modified by Galactic absorption. The distributions of the source fluxes in the three bands are displayed in Figure 14; the median fluxes in the full, soft, and hard bands are $3.1 \times 10^{-16}, 6.5 \times 10^{-17}$, and $5.7 \times 10^{-16}$ erg $\mathrm{cm}^{-2} \mathrm{~s}^{-1}$, respectively. In Figure 15, we present the softband flux distributions for the $7 \mathrm{Ms}$ CDF-S, 4 Ms CDF-S (Xue et al. 2011), and 2 Ms CDF-S (Luo et al. 2008), respectively. It is clearly visible that significant numbers of new and fainter sources are detected with increased exposure times.

Applying the $K$ correction assuming a power-law spectrum and also correcting for Galactic absorption, we computed the apparent rest-frame $0.5-7.0 \mathrm{keV}$ luminosity $\left(L_{\mathrm{X}}\right)$ from, in order of priority, the observed full-band, soft-band, or hard-band flux; the luminosities derived from the fluxes in different energy bands are actually consistent with each other, as the same spectral shapes were adopted throughout all these calculations. These X-ray luminosities have not been corrected for any intrinsic absorption and hence are referred to as "apparent." We further used the Portable, Interactive, MultiMission Simulator (PIMMS) ${ }^{43}$ to estimate intrinsic absorption by assuming that the intrinsic power-law spectrum has a fixed photon index of 1.8 and any effective photon index smaller than this value is caused by intrinsic absorption. In this manner, we estimated intrinsic absorption column densities $\left(N_{\mathrm{H} \text {,int }}\right)$ for 701 sources, which range from $2.3 \times 10^{19} \mathrm{~cm}^{-2}$ to $1.9 \times 10^{24}$ $\mathrm{cm}^{-2}$ with a median value of $4.9 \times 10^{22} \mathrm{~cm}^{-2}$. For sources with effective photon indices greater than 1.8, the intrinsic absorption column densities were set to zero. With the estimated intrinsic column densities, the absorption-corrected intrinsic $0.5-7.0 \mathrm{keV}$ luminosities $\left(L_{\mathrm{X}, \text { int }}\right)$ were computed, and the correction factors range from 1-240 with a median value of 2.8 for the 701 sources having $N_{\mathrm{H} \text {,int }}>0$. The distribution of $L_{X, \text { int }}$ as a function of the source redshift is displayed in Figure 16. There are 613 sources having $L_{\mathrm{X} \text {,int }}>10^{42} \mathrm{erg} \mathrm{s}^{-1}$ and 108 sources having $L_{\mathrm{X} \text {,int }}>10^{44} \mathrm{erg} \mathrm{s}^{-1}$. The fluxes and luminosities are presented in Columns 64-69 of the maincatalog table; we did not compute X-ray luminosities for the 12 stars or the 22 sources that lack redshifts.

\footnotetext{
${ }^{43}$ http://cxc.harvard.edu/toolkit/pimms.jsp.
} 
Table 2

Summary of Chandra Source Detections

\begin{tabular}{lcccrr}
\hline \hline & \multirow{2}{*}{$\begin{array}{c}\text { Number of } \\
\text { Band }(\mathrm{keV})\end{array}$} & \multicolumn{3}{c}{ Detected Counts Per Source } \\
\cline { 2 - 6 } & Sources & 56916.2 & Minimum & Median & \multicolumn{1}{c}{$N M A D^{\mathrm{a}}$} \\
\hline Full $(0.5-7.0)$ & 916 & 38817.0 & 6.1 & $98.9 \pm 6.1$ & 104.4 \\
Soft $(0.5-2.0)$ & 871 & 18137.8 & 9.2 & $97.7 \pm 2.0$ & 47.6 \\
Hard (2-7) & 622 & $94.6 \pm 6.0$ & 90.6 & $343.5 \pm 65.9$ \\
\hline
\end{tabular}

Note.

${ }^{\text {a }}$ Normalized median absolute deviation, defined as $N M A D=1.48 \times \operatorname{median}(\mid$ counts - median(counts) $\mid)$.

Table 3

Sources Detected in One Band but not Another

\begin{tabular}{lcccc}
\hline \hline \multirow{2}{*}{$\begin{array}{l}\text { Detection Band } \\
\text { (keV) }\end{array}$} & \multicolumn{4}{c}{ Nondetection Energy Band } \\
\cline { 2 - 5 } & Full & Soft & Hard & Either \\
\hline Full $(0.5-7.0)$ & $\ldots$ & 129 & 302 & 22 \\
Soft $(0.5-2.0)$ & 84 & $\ldots$ & 364 & 84 \\
Hard $(2-7)$ & 8 & 115 & $\ldots$ & 8 \\
\hline
\end{tabular}

Note. For example, there were 129 sources detected in the full band, but not in the soft band, and there were 22 sources detected in the full band, but not in either the soft band or the hard band.

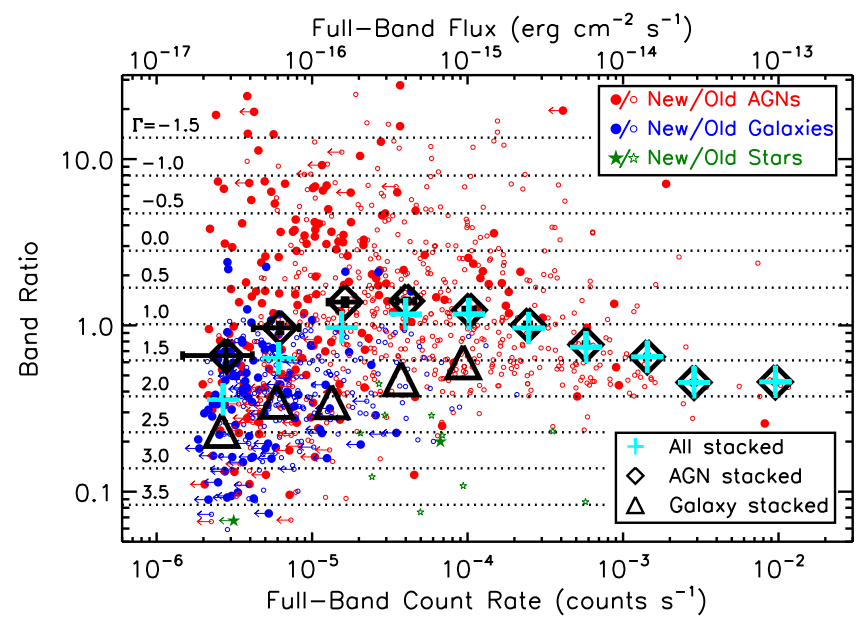

Figure 13. Band ratio vs. full-band count rate for the main-catalog sources. Sources having upper limits on the count rates are indicated by the arrows. Sources detected only in the full band (22 sources) are not included in this plot. Red, blue, and green symbols represent AGNs, galaxies, and stars, respectively. Filled symbols indicate newly detected sources, while open symbols are sources that were present in the Xue et al. (2011) 4 Ms CDF-S catalogs. The horizontal dotted lines show the band ratios corresponding to given effective photon indices; these were computed using the average of the XSPEC-derived $\Gamma_{\text {eff }}$-to-band-ratio conversions. The top $x$-axis displays the fullband fluxes at the corresponding count rates, derived assuming $\Gamma_{\text {eff }}=1.4$. The cyan plus signs represent the stacked count rates and band ratios for all of the sources within logarithmic count-rate bins of 0.4 , while the black diamonds and triangles are the stacked values for the AGNs and galaxies, respectively. The error bars for the stacked AGN data points are shown to illustrate the typical uncertainties of these stacked values; they become smaller than the symbol size in high count-rate bins.

\subsection{AGN Classification, Source Spatial Distribution, and Postage-stamp Images}

We classified AGNs from the detected X-ray sources by selecting sources having X-ray and/or multiwavelength properties significantly different from those of typical normal galaxies. Besides AGNs and Galactic stars, the other X-ray

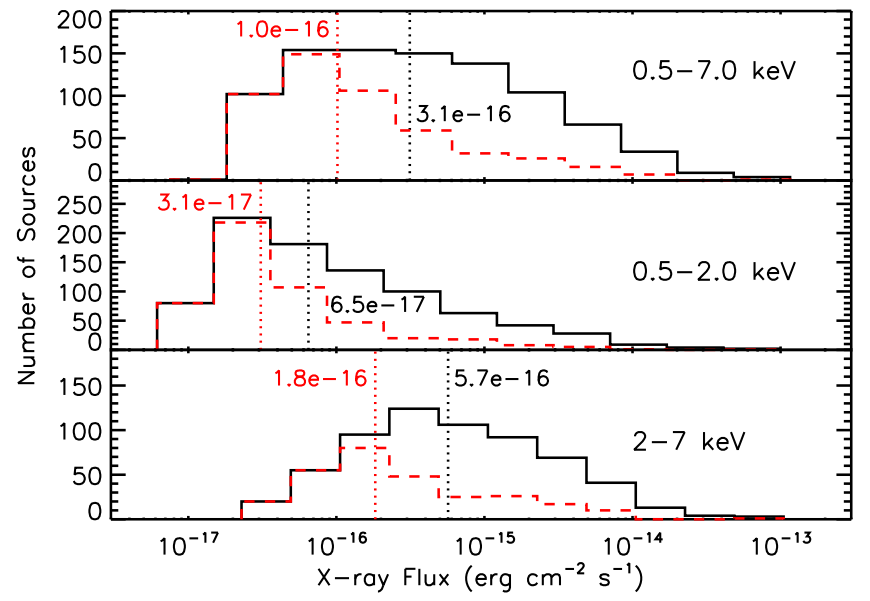

Figure 14. Distributions of X-ray fluxes for the main-catalog sources in the full (top), soft (middle), and hard (bottom) bands. The black-solid histograms show the flux distributions for all the sources in the CDF-S field, while the reddashed histograms show the distributions for the subgroups of sources within $6^{\prime}$ of the average aim point. Sources with upper limits on the fluxes are not included in the plots. The vertical dotted lines indicate the median fluxes for every distribution, and the corresponding median values are listed.

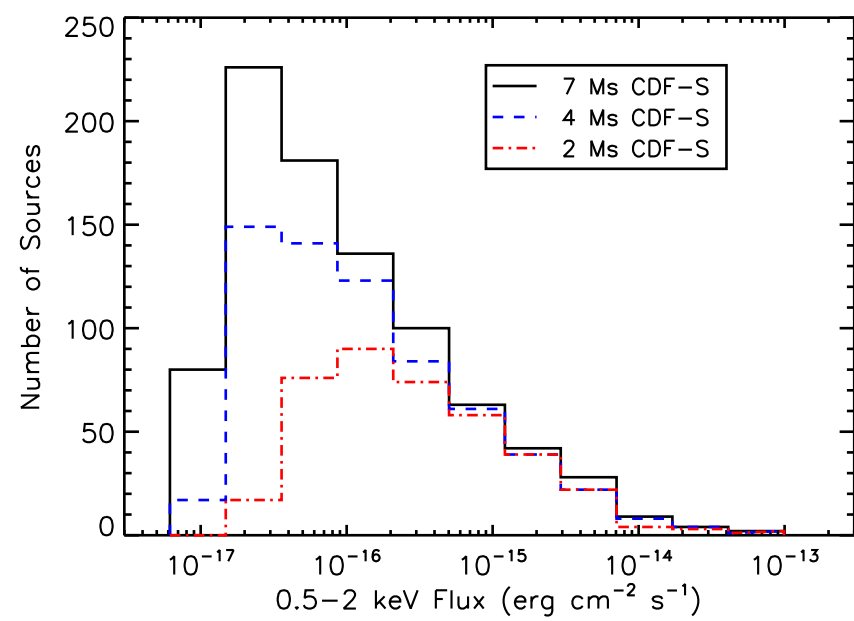

Figure 15. Soft-band flux distribution for the $7 \mathrm{Ms}$ CDF-S compared to those for the 4 Ms CDF-S (Xue et al. 2011) and 2 Ms CDF-S (Luo et al. 2008), showing the improvement of source detection from deeper exposures. The same flux binning was used for all three histograms.

sources are considered to be normal galaxies, although it is possible that some of these galaxies host low-luminosity and/ or heavily obscured AGNs where the AGN signatures are not evident based on our selection criteria; some of these missed AGNs could be identified by other means such as X-ray 


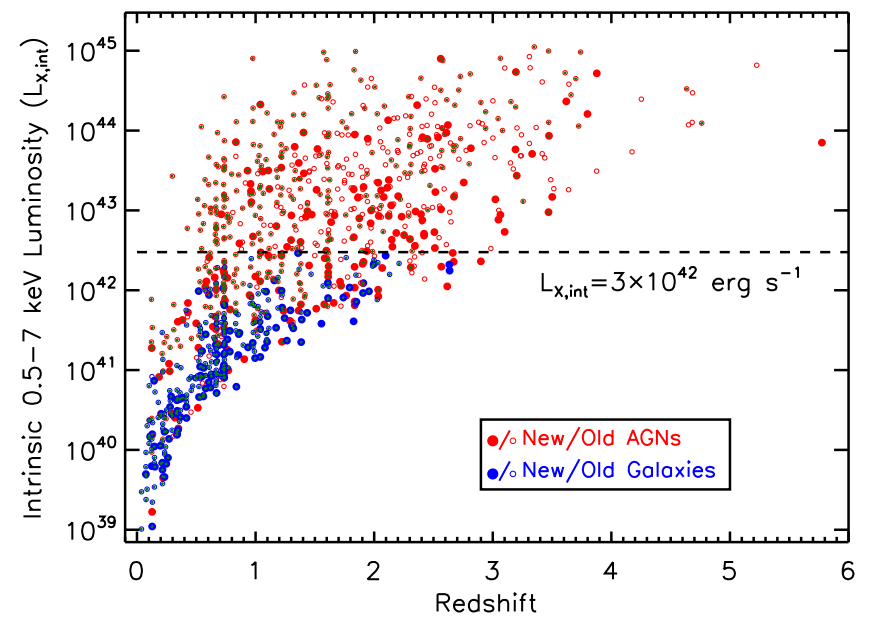

Figure 16. Intrinsic rest-frame $0.5-7.0 \mathrm{keV}$ luminosity (in erg s${ }^{-1}$ ) vs. redshift for the main-catalog sources. Red and blue symbols represent AGNs and galaxies, respectively; filled symbols indicate newly detected sources, while open symbols are sources that were present in the Xue et al. (2011) 4 Ms CDF$\mathrm{S}$ catalogs. Tiny green dots mark sources with spectroscopic redshifts. The horizontal dashed line indicates $L_{X}$,int $=3 \times 10^{42} \mathrm{erg} \mathrm{s}^{-1}$, which is one of the criteria utilized to classify AGNs. The 22 sources that lack redshifts and the 12 stars are not included in this plot.

variability (e.g., Young et al. 2012; P. Shao et al. 2017, in preparation).

We classified an X-ray source as an AGN if it satisfies one of the following six criteria: (1) $L_{\mathrm{X} \text {,int }} \geqslant 3 \times 10^{42} \mathrm{erg} \mathrm{s}^{-1}$ (luminous X-ray sources), (2) $\Gamma_{\text {eff }} \leqslant 1.0$ (hard X-ray sources), ${ }^{44}$ (3) X-rayto-optical flux ratio of $\log \left(f_{\mathrm{X}} / f_{R}\right)>-1$, where $f_{\mathrm{X}}$ is, in order of priority, the full-band, soft-band, or hard-band detected flux, and $f_{R}$ is the $R$-band flux, (4) spectroscopically classified as AGNs, (5) X-ray-to-radio luminosity ratio of $L_{X, \text { int }} / L_{1.4 \mathrm{GHz}} \geqslant 2.4 \times 10^{18}$, and (6) X-ray-to-NIR flux ratio of $\log \left(f_{\mathrm{X}} / f_{K s}\right)>-1.2$. The first five criteria were described in detail in Section 4.4 of Xue et al. (2011). For the last criterion, we chose an empirical threshold to classify X-ray excess sources as AGNs with the available AGN sample classified from the previous five criteria and the X-ray and TENIS $K_{s}$-band flux information (see Figure 17(b)); 12 new AGNs were classified based on this additional criterion.

In total, we identified 711 AGNs from the main catalog, and most $(86 \%)$ of them were classified based on two or more criteria. There are only five AGNs (XIDs 416, 494, 517, 523, 718) classified solely based on the first criterion $\left(L_{\mathrm{X}, \text { int }} \geqslant 3 \times 10^{42} \mathrm{erg} \mathrm{s}^{-1}\right)$. We caution that it is probable that the X-ray emission from some of these five sources could instead come from intense star formation (star formation rate $\gtrsim 300 M_{\odot} \mathrm{yr}^{-1}$; e.g., Lehmer et al. 2016); however, their estimated star formation rates are only $\approx 0.1-70 M_{\odot} \mathrm{yr}^{-1}$ (Skelton et al. 2014). Excluding the 12 stars, the remaining 285 sources are considered as normal galaxies (including the few off-nuclear sources). The distributions of the X-ray fluxes versus WFI $R$-band magnitudes and TENIS $K_{s}$-band magnitudes (the third and sixth classification criteria) for the maincatalog sources are displayed in Figure 17, and the regions expected to be occupied by AGNs are highlighted. The X-ray

\footnotetext{
${ }^{44}$ For the 479 sources detected in either the soft or hard band but not both, the mode values of the band ratios from BEHR were adopted which were then converted to $\Gamma_{\text {eff }}$. To ensure reliable AGN identification, we did not use these $\Gamma_{\text {eff }}$ values for the AGN selection. Instead, for a source to be classified as an AGN based on its $\Gamma_{\text {eff }}$, we required the source to be detected in the hard band and the $90 \%$ confidence-level upper limit on $\Gamma_{\text {eff }}$ to be less than 1, which was derived from the lower limit on the band ratio.
}

source classification is presented in Column 70 of the maincatalog table.

Figure 18(a) shows the spatial distribution of the maincatalog sources, which are color-coded as AGNs, galaxies, and stars. Figure 18(b) displays the observed source sky density as a function of the off-axis angle. These apparent source densities have not been corrected for detection incompleteness or Eddington bias; the number-count results, taking into account these effects, are presented in Section 8 below. The source densities decrease at larger off-axis angles due to the sensitivity degradation in the outer regions (e.g., see Section 7). In Figure 19, we show "postage-stamp" images for the maincatalog sources overlaid with adaptively smoothed X-ray contours. The images are color composites of the MUSYC $B$ band, WFI $R$-band, and TENIS $J+K_{s}$-band images. The X-ray contours were created using, in order of priority, the full-band, soft-band, or hard-band smoothed X-ray image in which the source is detected, and the wide range of source sizes represents the PSF broadening with off-axis angle. The source classification, adopted redshift, and net source counts are also indicated in each image. In Figure 20, we show postage-stamp images for the main-catalog sources that have GOODS-S and CANDELS coverage. The images are color composites of the GOODS-S $b$-band, GOODS-S $z$-band, and CANDELS F160W-band images.

\subsection{Comparison with the $4 \mathrm{Ms} C D F-S$ and Other Source Catalogs}

We matched the main-catalog sources to sources in the $4 \mathrm{Ms}$ CDF-S main and supplementary catalogs (Xue et al. 2011). A matching radius of $4^{\prime \prime}$ was used, and we visually inspected the $\mathrm{X}$-ray images for the unrecovered $4 \mathrm{Ms}$ sources and included two additional matches at large off-axis angles where the positional offsets are $\approx 4-6^{\prime \prime}$. The $7 \mathrm{Ms}$ CDF-S main source catalog recovered 704 of the $7404 \mathrm{Ms}$ main-catalog sources and 13 of the $364 \mathrm{Ms}$ supplementary catalog sources. Of the 36 $4 \mathrm{Ms}$ CDF-S main-catalog sources that are not present in the $7 \mathrm{Ms}$ main catalog, 10 are included in the $7 \mathrm{Ms}$ CDF-S supplementary catalog. We inspected the remaining 26 missing 4 Ms sources in detail, and they belong to one of the following four categories:

1. Nine sources have no multiwavelength counterparts, and they are weak X-ray sources with $\approx 8-60$ detected counts in the $4 \mathrm{Ms}$ CDF-S catalog. ${ }^{45}$ These are likely false detections in the $4 \mathrm{Ms}$ CDF-S.

2. Seven sources are located at large off-axis angles (7-11'), and have a companion X-ray source nearby (within 3.5-10"), which is detected in the $7 \mathrm{Ms}$ CDF-S (i.e., two $\mathrm{X}$-ray sources in the $4 \mathrm{Ms} \mathrm{CDF}-\mathrm{S}$ and only one in the $7 \mathrm{Ms}$ CDF-S). They have $\approx 16-130$ detected counts in the 4 Ms CDF-S catalog, and two of them have no multiwavelength counterparts. None of these seven offaxis sources were detected in the $250 \mathrm{ks}$ E-CDF-S catalogs (Xue et al. 2016). Most of these sources are likely false detections in the $4 \mathrm{Ms}$ CDF-S introduced when a single off-axis source was detected in two X-ray bands at different positions (separated by $3.5-10^{\prime \prime}$ ) and was treated as two sources. A few of them might be real

\footnotetext{
45 The source with $\approx 60$ counts from $0.5-8.0 \mathrm{keV}$ is at an off-axis angle of $\approx 9^{\prime}$, and it is a weak detection considering the large PSF size in the outer region of the CDF-S $\left(P_{\mathrm{B}, 4 \mathrm{Ms}}=0.002\right)$.
} 

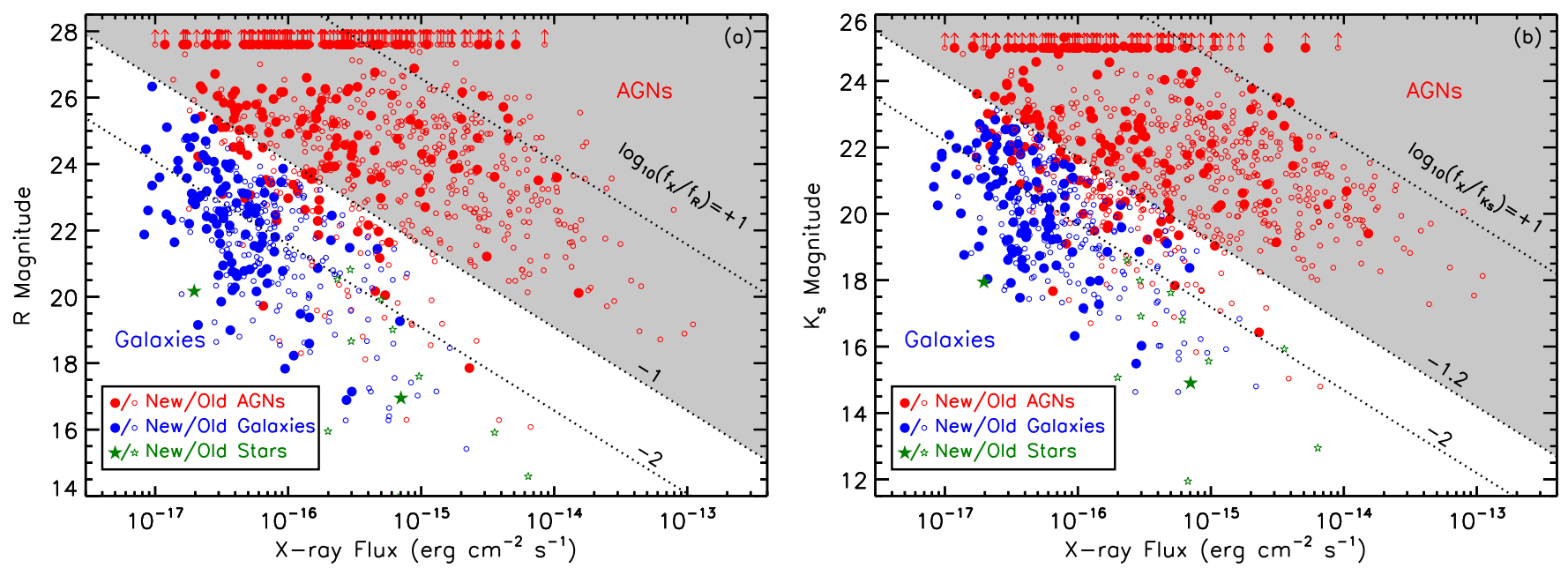

Figure 17. X-ray flux vs. (a) WFI $R$-band magnitude or (b) TENIS $K_{s}$-band magnitude for the main-catalog sources. We used, in order of priority, the full-band (91\%), soft-band $(8 \%)$, or hard-band $(1 \%)$ detected fluxes. Sources having limits on the magnitudes are indicated by the arrows. Red, blue, and green symbols represent AGNs, galaxies, and stars, respectively. Filled symbols indicate newly detected sources, while open symbols are sources that were present in the Xue et al. (2011) 4 Ms CDF-S catalogs. The diagonal lines show constant X-ray to $R$ - or $K_{s}$-band flux ratios. We adopted $\log \left(f_{\mathrm{X}} / f_{R}\right)>-1$ or $\log \left(f_{\mathrm{X}} / f_{K s}\right)>-1.2$ as two of the six criteria to classify AGNs; the AGN regions are shaded in both panels.
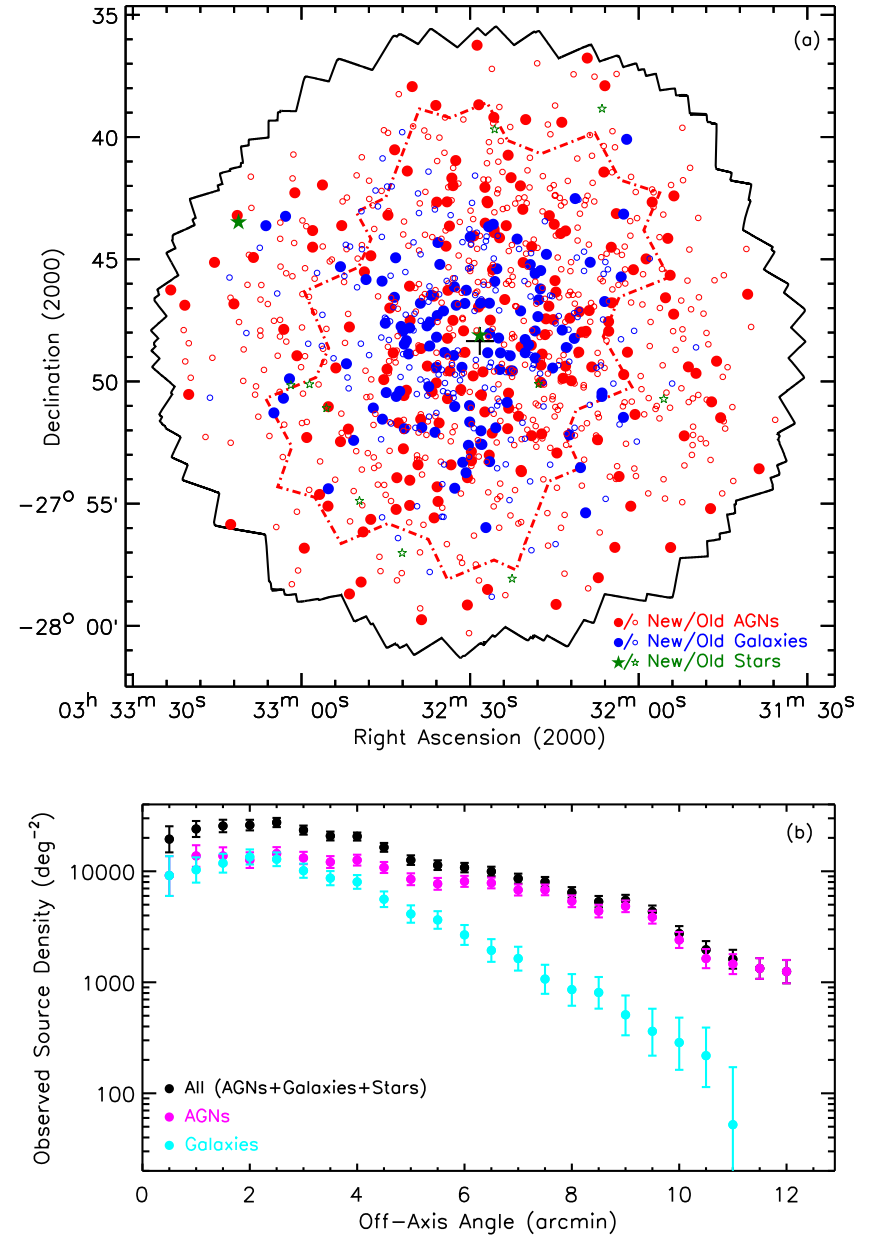

Figure 18. (a) Spatial distribution of the main-catalog sources. Red, blue, and green symbols represent AGNs, galaxies, and stars, respectively. Filled symbols indicate newly detected sources, while open symbols are sources that were present in the Xue et al. (2011) 4 Ms CDF-S catalogs. The average aim point, CDF-S boundary, and GOODS-S region are shown, as was done in Figure 1. (b) Observed X-ray source sky density as a function of off-axis angle in off-axis angle bins of $1^{\prime}$. The error bars are the $1 \sigma$ Poisson uncertainties (Gehrels 1986) on the source density in each bin. The AGN and galaxy density distributions are also shown. sources that are blended with the companion source in the $7 \mathrm{Ms}$ CDF-S

3. Eight sources have faint counterparts (GOODS-S $\left.z_{850} \approx 24-27\right)$ in the $4 \mathrm{Ms}$ catalog, and they have $\approx 8-40$ detected counts. They are not detected in the 7 Ms CDF-S probably due to source variability and/or background fluctuation. A few of these sources could also be false detections considering their counterparts are faint and the probability of a chance association is relatively high.

4. Two sources were detected by WAVDETECT in the $7 \mathrm{Ms}$ CDF-S, but they did not pass the $P_{\mathrm{B}}$ threshold cut, and their counterparts are not sufficiently bright to be included in the $7 \mathrm{Ms}$ supplementary catalog. They have 26 and 34 detected counts in the 4 Ms catalog, respectively. Their nature is similar to those sources in category (3).

In total, $\approx 16$ of the 26 missing $4 \mathrm{Ms}$ CDF-S main-catalog sources are probably spurious detections, which constitute $\approx 2 \%$ of the $4 \mathrm{Ms}$ CDF-S main catalog. The expected total number of spurious detections was $\approx 12$ in Section 6.2 of Xue et al. (2011), consistent with our assessment here. Of the 23 $(36-13=23)$ missing $4 \mathrm{Ms}$ CDF-S supplementary catalog sources, one is included in the $7 \mathrm{Ms}$ CDF-S supplementary catalog, and the other 22 are likely real sources that fall below the $7 \mathrm{Ms}$ CDF-S detection threshold due to source variability and/or background fluctuations (e.g., affected by Eddington bias); a minor fraction of them could be spurious detections.

There are 291 sources in the main catalog that are new detections compared to the $4 \mathrm{Ms}$ CDF-S catalogs. The new sources are distributed over the entire CDF-S field (Figure 18), and they are noted in the postage-stamp images (Figure 19). Three of the new sources (XIDs 528, 996, 1008) lie outside the footprint of the $4 \mathrm{Ms}$ CDF-S; they are all luminous AGNs and were detected in the $250 \mathrm{ks}$ E-CDF-S catalogs (Lehmer et al. 2005; Xue et al. 2016). We present the fractions of new sources at different off-axis angles in Figure 21, excluding the three new sources outside the $4 \mathrm{Ms}$ CDF-S footprint. The fraction of new sources is $\approx 40 \%$ in the field center, and it 


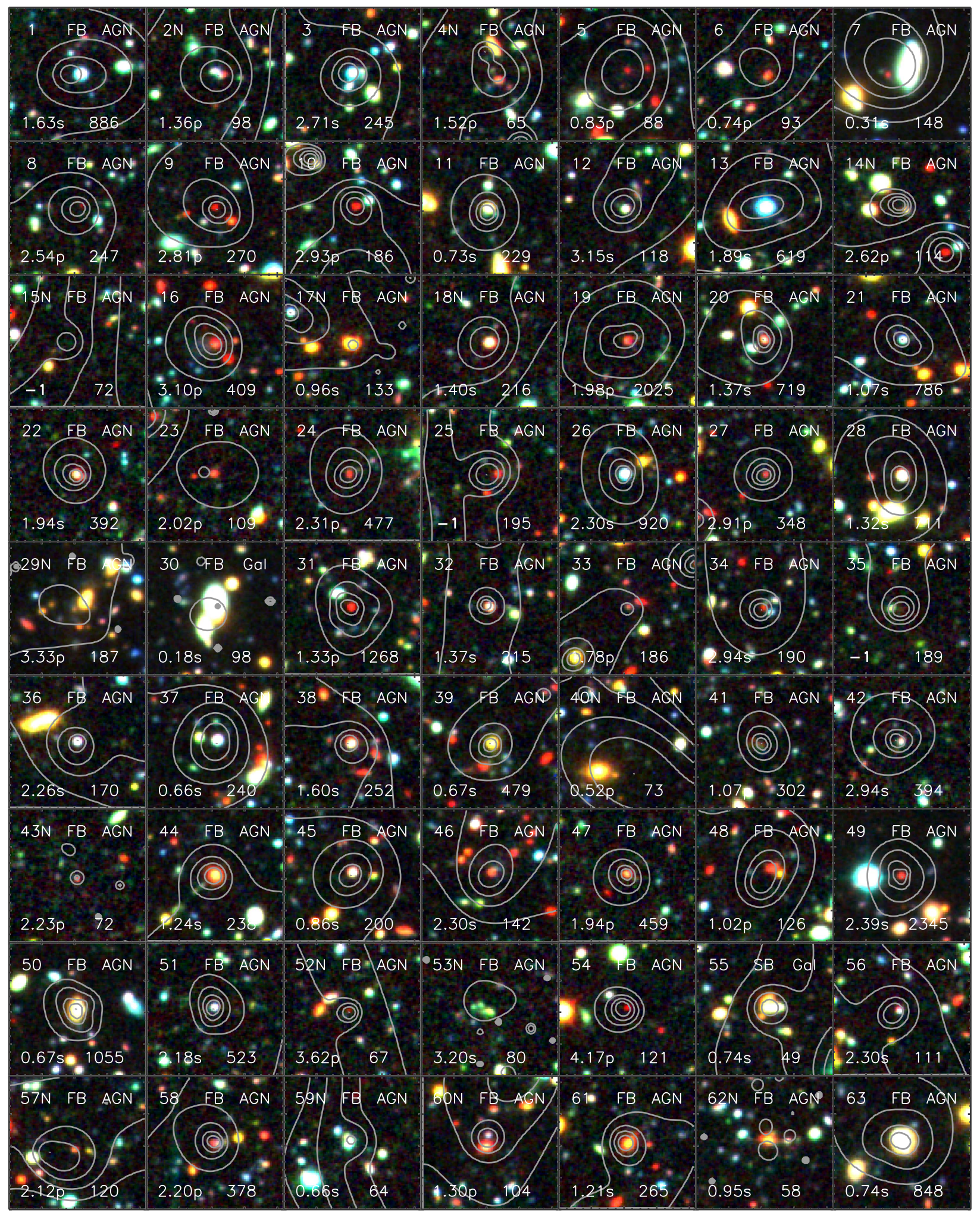

Figure 19. Postage-stamp images for the main-catalog sources overlaid with adaptively smoothed X-ray contours. The images are color composites of the MUSYC $B$ band (blue), WFI $R$-band (green), and TENIS $J+K_{s}$-band (red) images. Each image is $25^{\prime \prime}$ on a side, with the X-ray source located at the center. The X-ray contours were created using, in order of priority, the full-band, soft-band, or hard-band smoothed X-ray image $\left(25^{\prime \prime} \times 25^{\prime \prime}\right)$ in which the source is detected, and they are logarithmically scaled. If a source is faint (thus not apparent in the smoothed image) or if there is a brighter source nearby (the contour levels centered on the brighter source), there may be no X-ray contours around the image center. The main-catalog ID number (a letter " $\mathrm{N}$ " is attached if the source is newly detected compared to the 4 Ms CDF-S catalogs), X-ray band, and source classification (AGN, galaxy, or star) are given at the top of each image; the numbers at the bottom are the adopted redshift ("-1" if not available; marked with "s" if it is a spectroscopic redshift or "p" if a photometric redshift) and the net source counts in the corresponding X-ray band. Only the first page is shown here for illustrative purposes; the entire set of 16 pages of images is available in the online version of the journal.

(An extended version of this figure is available.) 


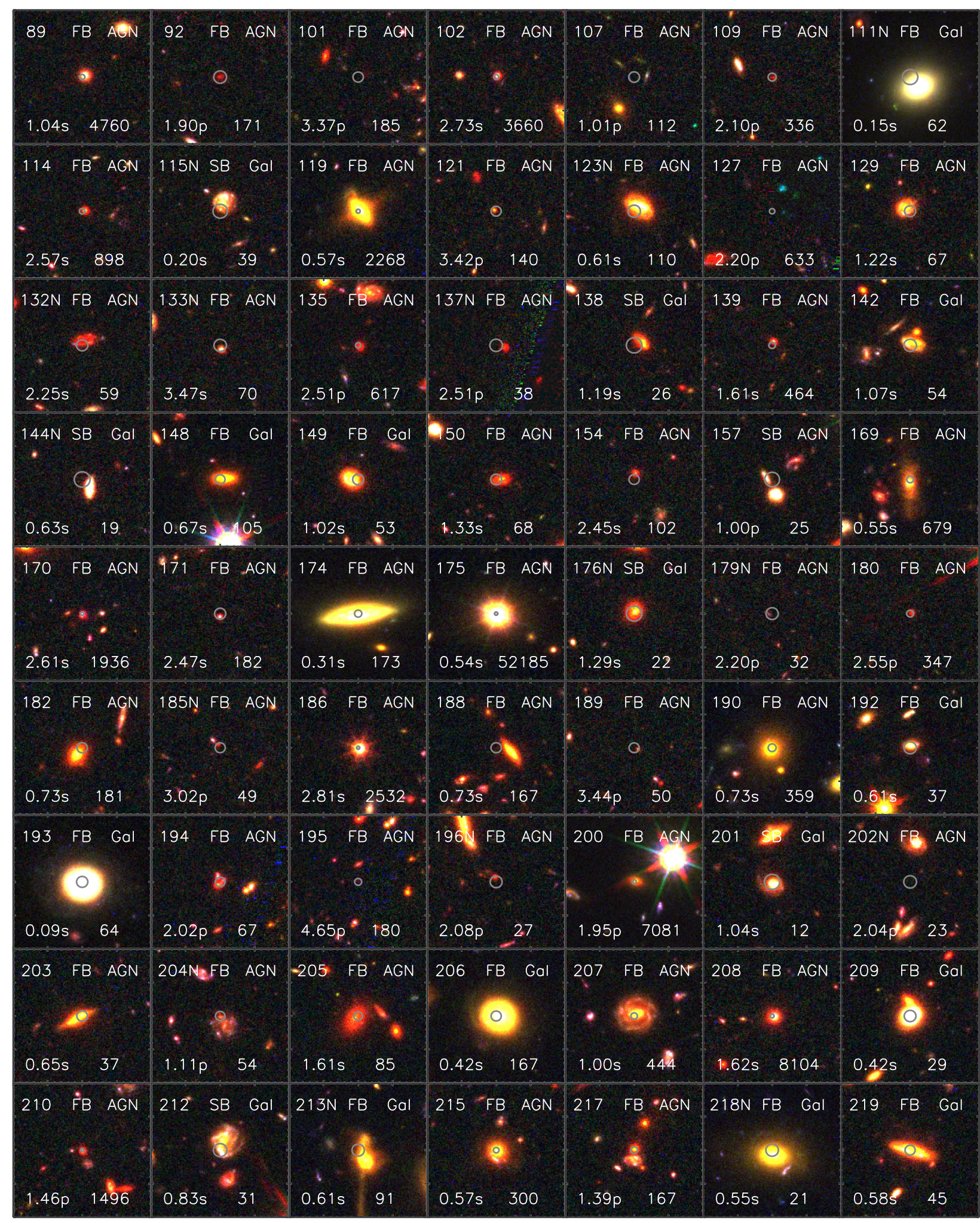

Figure 20. Postage-stamp images for the main-catalog sources that have GOODS-S and CANDELS coverage. The images are color composites of the GOODS-S $b$ band (blue), GOODS-S $z$-band (green), and CANDELS F160W-band (red) images. Each image is $12^{\prime \prime}$ on a side, with the X-ray source indicated by a small central circle of radius equal to the $1 \sigma \mathrm{X}$-ray positional uncertainty. The main-catalog ID number (a letter "N" is attached if the source is newly detected compared to the 4 Ms CDF-S catalogs), X-ray band, and source classification (AGN, galaxy, or star) are given at the top of each image; the numbers at the bottom are the adopted redshift ("-1" if not available; marked with "s" if it is a spectroscopic redshift or "p" if a photometric redshift) and the net source counts in the corresponding X-ray band. Only the first page is shown here for illustrative purposes; the entire set of 11 pages of images is available in the online version of the journal.

(An extended version of this figure is available.) 


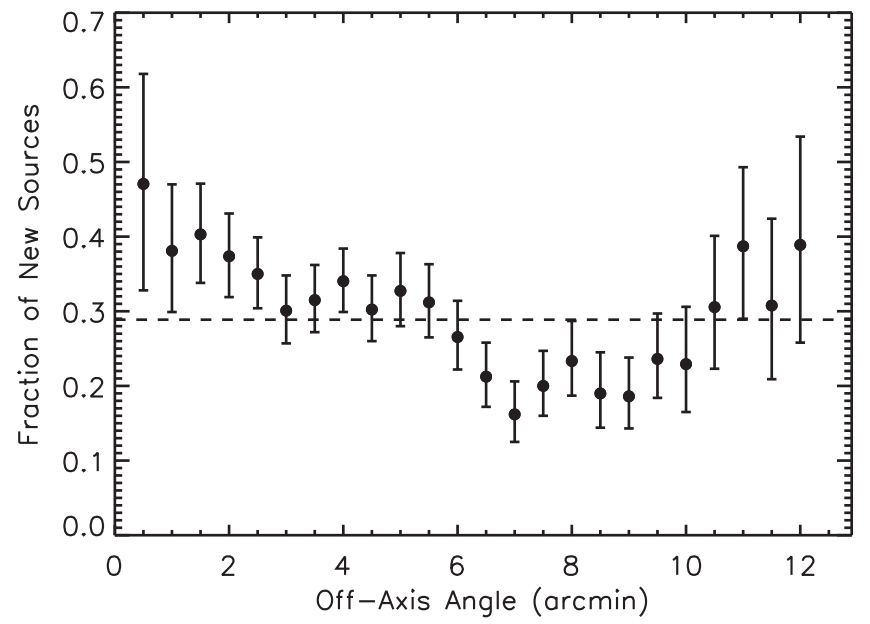

Figure 21. Fraction of new sources as a function of off-axis angle in off-axis angle bins of $1^{\prime}$, excluding the three new sources outside the 4 Ms CDF-S footprint. The error bars are the $1 \sigma$ Poisson uncertainties (Gehrels 1986). The horizontal dashed line represents the total fraction of new sources $(291 / 1008=29 \%)$. The rise of the new-source fraction beyond an off-axis angle of $\approx 10^{\prime}$ is likely caused by the dominating population of new sources detected in the more sensitive 2-7 keV band adopted in the $7 \mathrm{Ms}$ CDF-S rather than the $2-8 \mathrm{keV}$ band in the $4 \mathrm{Ms}$ CDF-S.

decreases to $\approx 20 \%$ at large off-axis angles; this behavior is likely due to the greater sensitivity improvement in the central region from the $4 \mathrm{Ms}$ CDF-S to the $7 \mathrm{Ms}$ CDF-S (see Section 7.3 of Xue et al. 2011). Beyond an off-axis angle of $\approx 10^{\prime}$, there is a weak rise of the new-source fraction toward larger off-axis angles. Most of the new sources in this outer region are detected in the hard band $(18 / 21=86 \%)$, while the hard-band detection fraction among all the 291 new sources is only $42 \%$. One possible explanation is that the new sources detected in the outer region are largely due to the more sensitive $2-7 \mathrm{keV}$ band adopted in the $7 \mathrm{Ms} \mathrm{CDF}-\mathrm{S}$, rather than the $2-8 \mathrm{keV}$ band in the $4 \mathrm{Ms}$ CDF-S (see Footnote 16 of Xue et al. 2016 and our sensitivity discussion in Section 7 below). Although there are also such new sources detected at smaller off-axis angles, the source number is smaller than the number of new sources detected due to improved sensitivity (increased exposure). They only become a dominant population at large off-axis angles where the number of new sources detected due to increased exposure is small, and this extra population of new sources causes the rise of the new-source fraction.

In terms of source classification, a smaller fraction of the new sources are AGNs $(184 / 291=63 \% \pm 5 \%)$ compared to that for the entire catalog $(711 / 1008=71 \% \pm 3 \%)$, indicating the rise of galaxy population toward lower X-ray fluxes (e.g., Bauer et al. 2004; Ranalli et al. 2005; Lehmer et al. 2012; Section 8 below). There are also two new stars detected. The $P_{\mathrm{B}}$ distribution for the new sources is shown in Figure 22. Compared to the distribution for the entire catalog (Figure 8), the new sources are less significantly detected with larger $P_{\mathrm{B}}$ values overall. Most of the sources without multiwavelength counterparts (14 out of the 16) are new sources and are likely to be spurious detections.

The redshift distributions for the new AGNs and galaxies are displayed in Figure 23. The median redshifts for the new sources do not differ from those for the entire AGN and galaxy samples (Figure 9(a)) after accounting for the uncertainties. The source-count and flux distributions for the 291 new sources

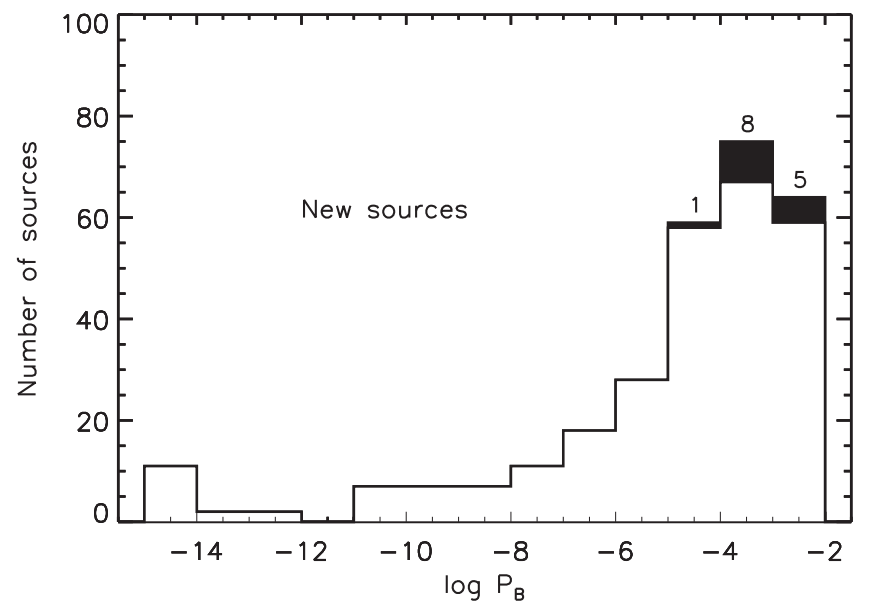

Figure 22. Similar to Figure 8, but for the 291 new sources, showing the distribution of the AE binomial no-source probability $\left(P_{\mathrm{B}}\right)$. The shaded regions indicate sources that have no multiwavelength counterparts, with the numbers of such sources shown on the top of each bin.

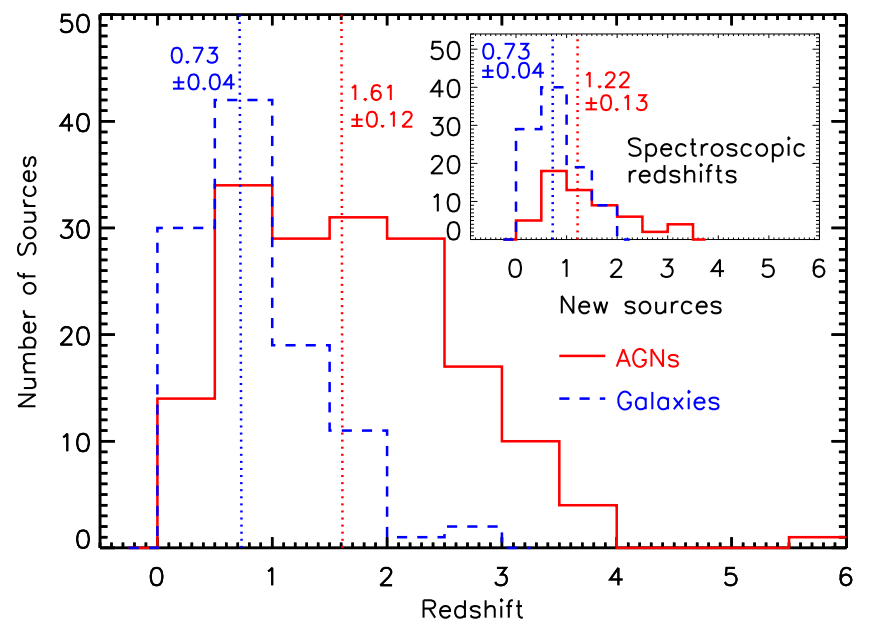

Figure 23. Similar to Figure 9(a), but for the new sources, showing the redshift distributions. The vertical dotted lines indicate the median redshift for every distribution, and the corresponding median values and their $1 \sigma$ uncertainties (derived via bootstrapping) are listed. The median redshifts for the new AGNs and galaxies are consistent with those for the entire AGN and galaxy samples (Figure 9(a) within the uncertainties.

are shown in Figure 24. Compared to the distributions for the entire catalog (Figures 12 and 14), the newly detected sources have fewer source counts and lower X-ray fluxes, regardless of their location in the field. The new sources and the sources already present in the $4 \mathrm{Ms}$ CDF-S catalogs ("old sources") are represented by different symbols in the luminosity versus redshift plot (Figure 16). The new sources appear to be less luminous overall. To better compare the X-ray fluxes and luminosities between the new and old sources, we present flux and luminosity histograms for the new and old AGNs and galaxies in Figure 25. The new AGNs in the main catalog have a lower median flux and median luminosity than the old AGNs. The new galaxies have a slightly lower median flux than the old galaxies, while their median luminosities are comparable.

The new AGNs appear to be more X-ray absorbed than the old AGNs. The median absorption column density estimates for the new and old AGNs are $(7.0 \pm 1.8) \times 10^{22} \mathrm{~cm}^{-2}$ and $(4.2 \pm 0.6) \times 10^{22} \mathrm{~cm}^{-2}$, respectively. A Kolmogorov-Smirnov (K-S) test suggests that the two column-density 

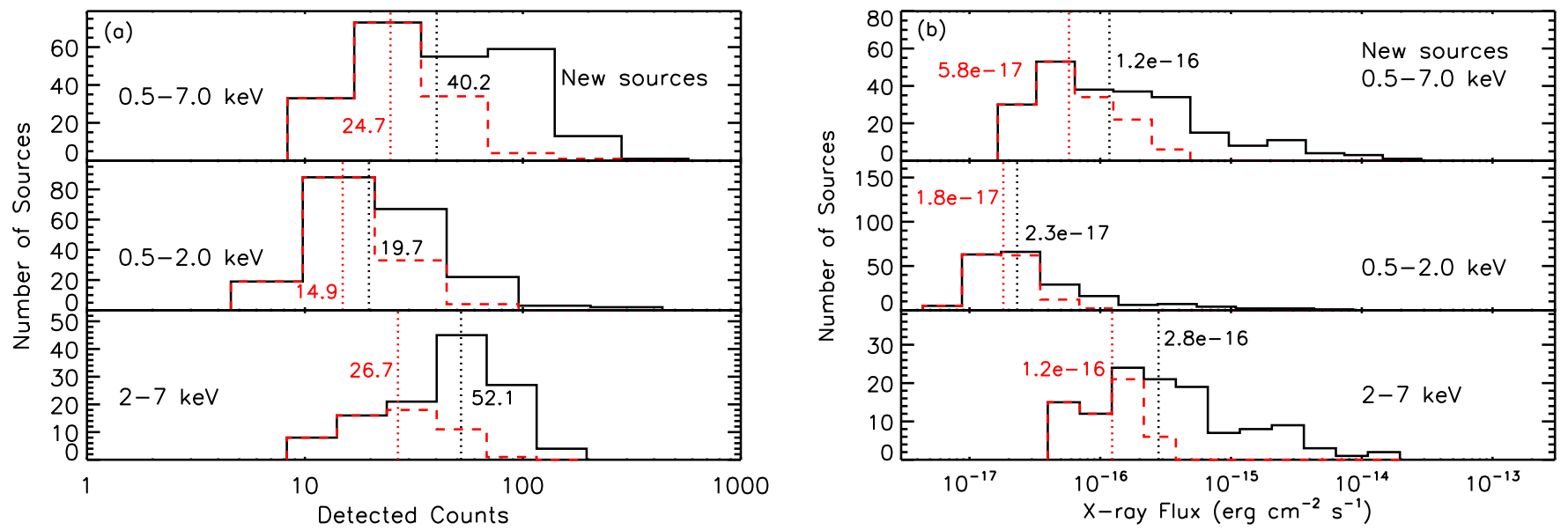

Figure 24. (a) Similar to Figure 12, but for the new sources, showing the distributions of detected source counts. The black-solid histograms show the count distributions for all the sources in the CDF-S field, while the red-dashed histograms show the distributions for the subgroups of sources within $6^{\prime}$ of the average aim point. The vertical dotted lines indicate the median numbers of counts for every distribution, and the corresponding median values are listed. (b) Similar to Figure 14, but for the new sources, showing the distributions of X-ray fluxes. The black-solid histograms show the flux distributions for all the sources in the CDF-S field, while the red-dashed histograms show the distributions for the subgroups of sources within $6^{\prime}$ of the average aim point. The vertical dotted lines indicate the median fluxes for every distribution, and the corresponding median values are listed.
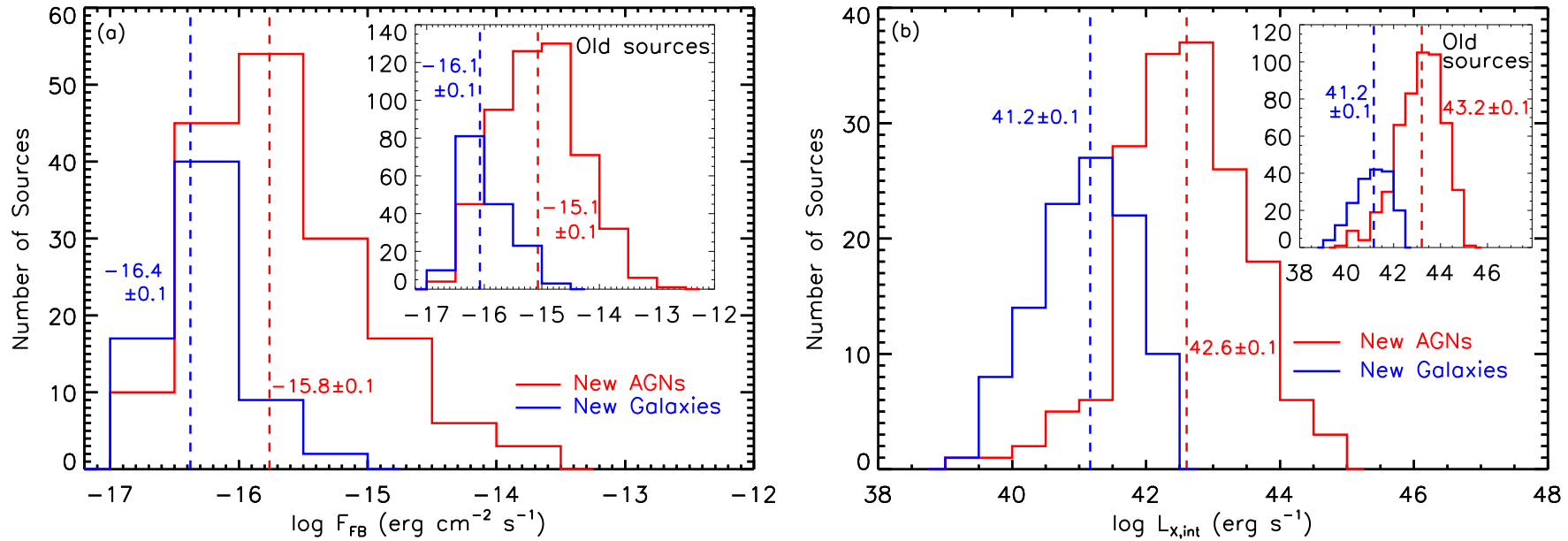

Figure 25. Distributions of (a) full-band fluxes and (b) intrinsic $0.5-7.0 \mathrm{keV}$ luminosities for the newly detected main-catalog sources. In each panel, the inset displays the corresponding distributions for the main-catalog sources that were present in the Xue et al. (2011) 4 Ms CDF-S catalogs. The red and blue histograms indicate the distributions for AGNs and galaxies, respectively. The vertical dashed lines show the median values of the corresponding distributions, and the numbers display the corresponding median values and their $1 \sigma$ uncertainties (derived via bootstrapping). Sources with upper limits on the full-band fluxes (92 sources; $9 \%$ ) are not included in panel (a). The 22 sources that lack redshifts and the 12 stars are not included in panel (b).

distributions differ significantly, with a $\mathrm{K}-\mathrm{S}$ probability of 0.009 . We caution that the absorption column densities were estimated with simplistic assumptions (Section 4.4$)^{46}$ and may be not reliable for such statistical comparisons.

We matched the main-catalog sources to sources in the $250 \mathrm{ks}$ E-CDF-S main and supplementary catalogs (Xue et al. 2016) using a matching radius of $4^{\prime \prime}$. One additional off-axis match with an offset of 5".9 was found via visual inspection. In total, 307 main-catalog sources were detected in the E-CDF-S main and supplementary catalogs. We also matched the main-catalog sources to sources in the 3 Ms XMMNewton CDF-S catalogs (Ranalli et al. 2013). For each maincatalog source, an XMM-Newton counterpart was searched for within a radius that is three times the quadratic sum of the $1 \sigma$ Chandra and XMM-Newton positional errors. In total, 292

\footnotetext{
46 The spectral shapes $\left(\Gamma_{\text {eff }}\right)$ and thus intrinsic absorption column densities are especially uncertain for the 479 sources detected in either the soft or hard band but not both, where the best-guess estimates of the band ratios were adopted.
}

main-catalog sources have XMM-Newton counterparts. There are cases where multiple Chandra sources were matched to a single XMM-Newton source (i.e., the XMM-Newton counterpart IDs for different 7 Ms CDF-S sources are the same), probably due to source blending in the XMM-Newton catalogs. The counterpart IDs in the 4 Ms CDF-S, $250 \mathrm{ks}$ E-CDF-S, and 3 Ms XMM-Newton CDF-S are presented in Columns 71-73 of the main-catalog table.

There have also been specialized searches for faint X-ray sources in the CDF-S using optical/NIR source positions as priors (e.g., Fiore et al. 2012; Giallongo et al. 2015; Cappelluti et al. 2016). We do not compare our source catalogs to those detections, due to the significantly different source-detection approaches adopted.

There are 19 main-catalog sources having XMM-Newton counterparts but no $4 \mathrm{Ms}$ CDF-S or $250 \mathrm{ks}$ E-CDF-S counterparts. Most (15) of these XMM-Newton counterparts were also matched to other Chandra sources (i.e., multiple Chandra sources matched to a single XMM-Newton source), and they 
were detected in the $4 \mathrm{Ms}$ CDF-S or $250 \mathrm{ks}$ E-CDF-S (i.e., not new sources detected by XMM-Newton). However, there are four main-catalog sources (XIDs 4, 571, 942, 954) with $X M M-N e w t o n$ counterparts that appear to be missed by the $4 \mathrm{Ms}$ CDF-S and $250 \mathrm{ks}$ E-CDF-S catalogs. Three of them (except XID 954) are hard X-ray sources with $\Gamma_{\text {eff }} \approx-1.5-0.3$, and they are at large off-axis angles $\left(\approx 7-11^{\prime}\right)$ detected with 66-156 full-band counts in the $7 \mathrm{Ms}$ CDF-S; these qualities help to explain why they were missed by previous Chandra surveys but were detected by XMM-Newton. The $7 \mathrm{Ms}$ CDF-S hard-band (2-7 keV) fluxes for these three sources are consistent with the $2-10 \mathrm{keV} X M M-N e w t o n$ fluxes within a factor of $\approx 1-2 .^{47}$ The other source, XID 954, is at an off-axis angle of $9 ! 2$ and detected with $\approx 70$ full-band and $\approx 60$ softband counts in the $7 \mathrm{Ms}$ CDF-S. The effective exposure time in the $4 \mathrm{Ms}$ CDF-S at the source location is $\approx 45 \%$ of that in the $7 \mathrm{Ms}$ CDF-S, which likely explains the nondetection of this weak off-axis source in the $4 \mathrm{Ms}$ CDF-S. However, in the $7 \mathrm{Ms}$ CDF-S, XID 954 was not detected in the hard band with an upper limit on the $2-7 \mathrm{keV}$ flux of $2.5 \times 10^{-16} \mathrm{erg} \mathrm{cm}^{-2} \mathrm{~s}^{-1}$, while in the XMM-Newton catalog, it has no signal in the $0.5-2.0 \mathrm{keV}$ band and the $2-10 \mathrm{keV}$ measured flux is $3.0 \times 10^{-15} \mathrm{erg} \mathrm{cm}^{-2} \mathrm{~s}^{-1}$. It was not classified as an AGN in the $7 \mathrm{Ms}$ CDF-S, and the X-ray emission likely originates from a $z=0.129$ galaxy with $L_{X}=1.6 \times 10^{40} \mathrm{erg} \mathrm{s}^{-1}$. The substantial hard X-ray signal in the $3 \mathrm{Ms} X M M-N e w t o n$ data probably came from some outburst phenomenon, for example, an obscured stellar tidal disruption event or a heavily obscured AGN revealing temporarily some of its hard X-ray emission due to reduced absorption. The nature of this source is worth further investigation. Two other candidate new sources proposed in the $3 \mathrm{Ms} X M M-N e w t o n$ CDF-S catalogs (Section 8.4 of Ranalli et al. 2013), with XMM-Newton IDs 85 and 1149 , remain undetected in the $7 \mathrm{Ms}$ CDF-S.

\subsection{Notes on Individual Objects}

\subsubsection{Variable Sources}

One of the main-catalog sources, XID 725, was discovered as a fast X-ray transient shortly after the relevant observation was taken (Luo et al. 2014a). Most $(\approx 90 \%)$ of the source counts $(\approx 120)$ arrived within a time span of $\approx 5 \mathrm{ks}$ during observation 16454 on 2014 October 1. The source did not produce any detectable X-ray emission before or after this transient event in the $7 \mathrm{Ms}$ CDF-S. The counterpart is a faint galaxy $(\mathrm{F} 125 \mathrm{~W}=27.1)$ at a photometric redshift of $\approx 2.1$. The nature of the source is unclear and detailed investigation is presented in F. E. Bauer et al. (2017, in preparation). Our source catalog was constructed using the merged $7 \mathrm{Ms}$ data, and thus the X-ray properties (e.g., flux and luminosity) of this source were averaged over the entire data set.

Another main-catalog source, XID 403, was discovered as a highly variable X-ray source during the first half of the new CDF-S observations (Luo et al. 2014b). It was not detected in the $4 \mathrm{Ms}$ CDF-S, yet it was significantly detected in the recent $3 \mathrm{Ms}$ CDF-S observations with $\approx 250$ full-band counts, brightening by a factor of $>13$. The counterpart is a $R=24.4$ galaxy at a photometric redshift of $\approx 1.5$. The $\mathrm{X}$-ray source is very soft and it was not detected in the hard

\footnotetext{
47 Considering statistical uncertainties, cross-calibration uncertainties, and the difference in the energy bands, there is no significant hard X-ray flux variability for these objects.
}

band $\left(\Gamma_{\text {eff }} \approx 3.0\right)$. The $\mathrm{X}$-ray light curve shows a gradual decline over the time span of years. This X-ray source is probably associated with a stellar tidal disruption event. If confirmed, it is the highest redshift tidal disruption event discovered so far, owing to the exceptionally deep exposure of the $7 \mathrm{Ms}$ CDF-S. Another possibility is that it is an AGN that varies strongly on long timescales (e.g., PHL 1092; Miniutti et al. 2012). Detailed investigation of this source is presented in W. Wang et al. (2017, in preparation). The X-ray properties provided in the main catalog (e.g., flux and luminosity) are the average values over the entire $7 \mathrm{Ms}$ data set.

\subsubsection{Sources without Multiwavelength Counterparts}

Among all the sources without multiwavelength counterparts, only one (XID 912) is highly significantly detected with a minimum WAVDETECT false-positive probability of $10^{-8}$ and an AE no-source probability of $P_{\mathrm{B}} \approx 10^{-13}$ (Figure 8); it is also present in the $4 \mathrm{Ms}$ CDF-S catalog. This source has 73.2 softband counts, and it was not detected in the hard band. The effective power-law photon index is $\approx 2.9$, and a simple powerlaw fit to the X-ray spectrum resulted in a photon index of $3.1 \pm 0.5$. The X-ray photons were relatively evenly distributed among the $7 \mathrm{Ms}$ observations, although some lowamplitude variability is apparent. If we compute the $P_{\mathrm{B}}$ value for each observation individually, the source would be considered detected in observations 582 and 8596, but not in the other observations. We inspected the multiwavelength images (Section 4.2) and did not find any radio/IR/NIR/ optical counterpart at the X-ray source position. The X-ray source is within the CANDELS coverage, but it is close to the edge of that field, and it lies outside the GOODS-S footprint. We examined the SEDS IRAC $5.8 \mu \mathrm{m}$ and the Far-Infrared Deep Extragalactic Legacy Survey (FIDEL) MIPS $24 \mu \mathrm{m}$ and $70 \mu \mathrm{m}$ images, and there is no apparent source at the X-ray source position. There is a bright $(R \approx 18)$, low-redshift (0.105) X-ray detected galaxy (XID 916) located 7!"5 away from this unidentified X-ray source. The X-ray photometry of the two sources is not affected significantly, as the separation allows $\approx 85 \%$ ECF source-extraction apertures to be used for both sources. The nature of this unidentified X-ray source remains unclear. One possible explanation is that it is an offnuclear source associated with the nearby galaxy, approximately $15 \mathrm{kpc}$ from the center, although the HST CANDELS image does not reveal such a large extent of the galaxy. Another possibility is that this is a high-redshift dusty AGN where the observed-frame NIR/IR emission (rest-frame optical/NIR) is heavily obscured, and it requires longerwavelength sensitive observations (e.g., ALMA) for a detection. The soft observed X-ray spectral shape is inconsistent with the latter scenario, but the limited X-ray photon statistics cause significant uncertainties in the estimated spectral shape.

\subsection{Main-Catalog Details}

We present the main Chandra source catalog in Table 4. The details of the table columns are given below.

1. Column 1: the source sequence number (XID) assigned in order of increasing R.A.

2. Columns 2 and 3: the R.A. and decl. of the source, respectively. See Section 3.2.

3. Column 4: the logarithm of the final $P_{\mathrm{B}}$ value. We set $\log P_{\mathrm{B}}=-99$ when $P_{\mathrm{B}}=0$. The threshold for being 
Table 4

Main Chandra Source Catalog

\begin{tabular}{|c|c|c|c|c|c|c|c|c|c|c|c|c|}
\hline $\begin{array}{l}\text { XID } \\
(1)\end{array}$ & $\begin{array}{l}\text { R.A. } \\
\text { (2) }\end{array}$ & $\begin{array}{c}\text { Decl. } \\
(3)\end{array}$ & $\underset{(4)}{\log P_{B}}$ & $\begin{array}{l}\text { WAVDETECT } \\
(5)\end{array}$ & $\begin{array}{l}\text { Pos Err } \\
\text { (6) }\end{array}$ & $\begin{array}{l}\text { Off-axis } \\
(7)\end{array}$ & $\begin{array}{l}\text { FB } \\
(8)\end{array}$ & $\begin{array}{c}\text { FB } \\
\text { Low Err } \\
(9)\end{array}$ & $\begin{array}{c}\text { FB } \\
\text { Upp Err } \\
(10)\end{array}$ & $\begin{array}{l}\text { SB } \\
(11)\end{array}$ & $\begin{array}{c}\text { SB } \\
\text { Low Err } \\
(12)\end{array}$ & $\begin{array}{c}\text { SB } \\
\text { Upp Err } \\
(13)\end{array}$ \\
\hline 1 & 52.899178 & -27.859588 & -99.0 & -8 & 0.53 & 12.04 & 886.7 & 38.2 & 39.3 & 604.3 & 28.2 & 29.4 \\
\hline 2 & 52.911023 & -27.892965 & -10.2 & -8 & 1.08 & 12.15 & 98.9 & 20.1 & 21.2 & 67.3 & 12.3 & 13.5 \\
\hline 3 & 52.917119 & -27.796253 & -99.0 & -8 & 0.66 & 10.67 & 245.2 & 22.0 & 23.1 & 152.6 & 14.9 & 16.1 \\
\hline 4 & 52.919726 & -27.773984 & -4.5 & -5 & 1.01 & 10.69 & 65.6 & 18.2 & 19.4 & 29.8 & -1.0 & -1.0 \\
\hline 5 & 52.920710 & -27.743110 & -14.4 & -8 & 0.97 & 11.12 & 88.7 & 13.8 & 15.0 & 32.3 & 7.5 & 8.7 \\
\hline
\end{tabular}

Note. The full table contains 73 columns of information for the 1008 X-ray sources.

(This table is available in its entirety in machine-readable form.)

included in the main catalog is $P_{\mathrm{B}}<0.007$. See Section 3.2.

4. Column 5: the logarithm of the minimum WAVDETECT false-positive probability, with $-8,-7,-6$, and -5 ranging from most significantly detected to least significantly detected. See Section 3.2.

5. Column 6: the $1 \sigma(\approx 68 \%$ confidence-level) positional uncertainty in units of arcseconds derived using Equation (2). The $90 \%$ and $95 \%$ confidence-level positional uncertainties are approximately 1.6 and 2.0 times the $1 \sigma$ positional uncertainty. See Section 4.1.

6. Column 7: the off-axis angle in units of arcminutes, which is the separation between the source position and the average aim point of the 7 Ms CDF-S (Section 2.1).

7. Columns 8-16: the aperture-corrected net source counts and the corresponding $1 \sigma$ lower and upper uncertainties in the full, soft, and hard bands, respectively. For sources undetected in a given band, the source-count column lists the $90 \%$ confidence-level upper limit on the source counts while the two associated uncertainty columns are set to "-1.0." See Section 4.4.

8. Column 17: photometric notes on individual sources. Sources covered by less than 20 of the 102 CDF-S observations are marked with "E," and sources in crowded regions are marked with "C." The other sources have this column set to "...." See Section 4.4.

9. Column 18: the catalog from which the primary counterpart was selected, being, in order of priority, one of the following six catalogs: CANDELS, GOODS-S, GEMS, TENIS, WFI, and SEDS. There are 710, 26, 187, 49, 4, and 16 primary counterparts from these six catalogs, respectively. The R.A., decl., and magnitude of the primary counterpart are included in Columns 21-41 below. Sources with no counterparts have this column set to "...." See Section 4.2.

10. Column 19: the positional offset between the X-ray source and the primary counterpart in units of arcseconds. Sources with no counterparts have this column set to " $-1.00 . "$

11. Column 20: counterpart notes on individual sources. Sources with their counterparts selected manually are marked with "Manual," sources matched to the same counterparts are marked with "Pair," sources that are candidates for being off-nuclear sources are marked with "Off-nuclear," and sources that are candidates for being extended jet/lobe emission are marked with "Jet." There are six sources marked as "Manual," three as "Manual
+Off-nuclear," one as "Off-nuclear," one as "Pair+Offnuclear," two as "Pair," one as "Pair+Manual+Offnuclear," one as "Pair+Jet," and one as "Pair+Offnuclear/Jet." The other sources have this column set to “...." See Section 4.2.

12. Columns 21-41: the R.A., decl., and magnitude of the counterparts in the WFI, GOODS-S, GEMS, CANDELS, TENIS, SEDS, and VLA catalogs, respectively. The AB magnitudes for the VLA $1.4 \mathrm{GHz}$ sources were converted from the radio flux densities $\left(m=-2.5 \log f_{\nu}-48.6\right)$. Sources with no counterparts in a given catalog have the corresponding columns set to "-1.00." See Section 4.2.

13. Columns 42-44: the spectroscopic redshift, quality flag ("Secure" or "Insecure"), and the catalog from which the redshift was collected (numbered 1-26; see Section 4.3 for the references). The spectroscopic redshifts for stars were set to zero. Sources without spectroscopic redshifts have these three columns set to " -1.000, , “...," and " -1 ," respectively. See Section 4.3.

14. Columns 45-50: the photometric redshifts from Luo et al. (2010), Rafferty et al. (2011), Hsu et al. (2014), Skelton et al. (2014), Santini et al. (2015), and Straatman et al. (2016), respectively. Sources that lack photometric redshifts in a given catalog have the corresponding column set to "-1.00." See Section 4.3.

15. Column 51: the adopted redshift. Sources that lack redshifts have this column set to " -1.00 ." See Section 4.3.

16. Column 52: the origin of the adopted redshift, being "zSpec" for spectroscopic redshifts and "L10," "R11," "H14," "S14," and "S16" for photometric redshifts from Luo et al. (2010), Rafferty et al. (2011), Hsu et al. (2014), Skelton et al. (2014), and Straatman et al. (2016), respectively. Sources that lack redshifts have this column set to "...." See Section 4.3.

17. Columns 53-54: the $1 \sigma$ lower and upper uncertainties on the adopted photometric redshifts. Sources that lack redshifts or have adopted spectroscopic redshifts have these columns set to "-1.00." See Section 4.3.

18. Columns 55-57: the effective exposure times derived from the exposure maps in the full, soft, and hard bands, respectively. See Section 3.1.

19. Columns 58-60: the X-ray band ratio and its $1 \sigma$ lower and upper uncertainties. Band ratios for sources detected in either the soft band or the hard band, but not both, are the mode values from BEHR (not upper or lower limits, but best-guess estimates) and the corresponding 
uncertainty columns were set to " -1.000 ." Sources detected only in the full band have these columns set to “-1.000." See Section 4.4.

20. Columns 61-63: the effective power-law photon index $\left(\Gamma_{\text {eff }}\right)$ and its $1 \sigma$ lower and upper uncertainties. Sources detected in either the soft band or the hard band, but not both, have their uncertainty columns set to " -1.00 ." We adopted $\Gamma_{\text {eff }}=1.4$ for sources detected only in the full band, and the uncertainty columns are set to " -1.00 ." See Section 4.4.

21. Columns 64-66: the X-ray fluxes in the full, soft, and hard bands, respectively. Negative values indicate $90 \%$ confidence-level upper limits on the fluxes that were derived from the upper limits on the source counts. See Section 4.4.

22. Column 67: the apparent rest-frame $0.5-7.0 \mathrm{keV}$ luminosity, which has not been corrected for any intrinsic absorption. Sources that lack redshifts or are identified as stars have this column set to "-1.00." See Section 4.4.

23. Column 68: the intrinsic absorption column density estimated based on the deviation between the effective photon index and the assumed intrinsic photon index of 1.8. Sources that lack redshifts or are identified as stars have this column set to "-1.00." See Section 4.4.

24. Column 69: the absorption-corrected intrinsic $0.5-7.0 \mathrm{keV}$ luminosity. Sources that lack redshifts or are identified as stars have this column set to "-1.00." See Section 4.4.

25. Column 70: the X-ray source type: "AGN," "Galaxy," or "Star." See Section 4.5.

26. Column 71: the matched 4 Ms CDF-S source ID number (Xue et al. 2011). A letter "S" is added to the ID number if the matched source is from the supplementary catalog. Sources that lack 4 Ms CDF-S counterparts have this column set to “...." See Section 4.6.

27. Column 72: the matched $250 \mathrm{ks}$ E-CDF-S source ID number (Xue et al. 2016). A letter " $\mathrm{S}$ " is added to the ID number if the matched source is from the supplementary catalog. Sources that lack $250 \mathrm{ks}$ E-CDF-S counterparts have this column set to "...." See Section 4.6.

28. Column 73: the matched 3 Ms XMM-Newton CDF-S source ID number (Ranalli et al. 2013). Sources that lack 3 Ms XMM-Newton CDF-S counterparts have this column set to " -1 ." See Section 4.6.

\section{SUPPLEMENTARY NEAR-INFRARED BRIGHT CHANDRA SOURCE CATALOG}

The supplementary source catalog contains information for the $47 \mathrm{X}$-ray sources that have $0.007 \leqslant P_{\mathrm{B}}<0.1$ and also bright $\left(K_{s} \leqslant 23\right)$ TENIS counterparts (Section 3.2). We created the supplementary catalog following the same procedures as for the main catalog, except that in the supplementary catalog, a source is considered to be detected when its $P_{\mathrm{B}}$ value is less than 0.1 (instead of 0.007 as in the main catalog). The X-ray positional uncertainties were calculated following Equation (2). The primary counterparts of the X-ray sources were set to be their TENIS counterparts, and we then searched for their optical through radio counterparts in the other multiwavelength catalogs (see Section 4.2). Thirty supplementary sources have spectroscopic redshifts, and another 16 have photometric redshifts from Hsu et al. (2014) or Straatman et al. (2016).

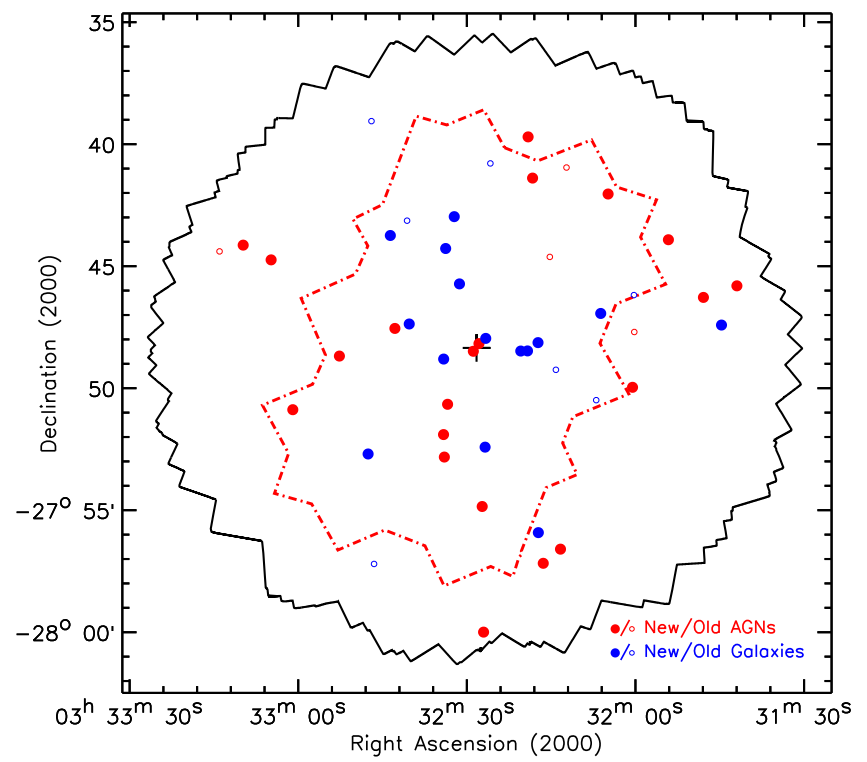

Figure 26. Spatial distribution of the supplementary catalog sources. Red and blue symbols represent AGNs and galaxies, respectively. Filled symbols indicate newly detected sources, while open symbols are sources that were present in the Xue et al. (2011) 4 Ms CDF-S catalogs. The average aim point, CDF-S boundary, and GOODS-S region are indicated, as was done in Figure 1.

The remaining supplementary source, supplementary XID 3, does not have a redshift estimate, as it lies outside the GOODS$S$ and CANDELS regions and it does not appear to have any optical counterparts, despite having a TENIS counterpart with $K_{s}=22.9$. The median redshift of the entire supplementary sample is $1.17 \pm 0.10$, similar to that for the main-catalog sources. There are 25 AGNs classified in the supplementary catalog, and the other 22 sources are likely normal galaxies. The fraction of AGNs in the supplementary catalog (25/ $47=53 \%)$ is smaller than that in the main catalog (711/ $1008=71 \%$ ), because fainter X-ray sources generally have a higher galaxy fraction (e.g., Bauer et al. 2004; Ranalli et al. 2005; Lehmer et al. 2012; Section 8 below), and our selection of supplementary sources is biased toward galaxies by requiring bright TENIS counterparts for the X-ray sources (e.g., see the sixth criterion of AGN classification in Section 4.5).

The spatial distribution of the supplementary sources is displayed in Figure 26. Compared to the Xue et al. (2011) 4 Ms CDF-S catalogs, 36 sources were newly detected, including 21 AGNs and 15 galaxies. We present the supplementary Chandra source catalog in Table 5; the details of the table columns are the same as those for the main catalog (Section 4.8).

\section{COMPLETENESS AND RELIABILITY ANALYSIS}

Given the nature of our two-stage source-detection approach, it is not straightforward to assess the completeness and reliability of our main source catalog. Therefore, we resorted to simulations for such an assessment, as done routinely among previous X-ray surveys (e.g., Cappelluti et al. 2007, 2009; Puccetti et al. 2009; Xue et al. 2011, 2016). We followed Section 6.1 of Xue et al. (2011) to generate simulated 7 Ms CDF-S observations with an input catalog of simulated sources; each observation has the same exposure time, aim point, roll angle, and aspect solution file as the corresponding real CDF-S observation. We then created images in the three X-ray bands from the merged simulated event file, and we ran WAVDETECT 
Table 5

Supplementary NIR Bright Chandra Source Catalog

\begin{tabular}{lccccccccccccc}
\hline \hline $\begin{array}{l}\text { XID } \\
(1)\end{array}$ & $\begin{array}{c}\text { R.A. } \\
(2)\end{array}$ & $\begin{array}{c}\text { Decl. } \\
(3)\end{array}$ & $\begin{array}{c}\log P_{\mathrm{B}} \\
(4)\end{array}$ & $\begin{array}{c}\text { WAVDETECT } \\
(5)\end{array}$ & $\begin{array}{c}\text { Pos Err } \\
(6)\end{array}$ & $\begin{array}{c}\text { Off-axis } \\
(7)\end{array}$ & $\begin{array}{c}\text { FB } \\
(8)\end{array}$ & $\begin{array}{c}\text { FB Low Err } \\
(9)\end{array}$ & $\begin{array}{c}\text { FB Upp Err } \\
(10)\end{array}$ & $\begin{array}{c}\text { SB } \\
(11)\end{array}$ & $\begin{array}{c}\text { SB Low Err } \\
(12)\end{array}$ & $\begin{array}{c}\text { SB Upp Err } \\
(13)\end{array}$ \\
\hline 1 & 52.925294 & -27.763536 & -1.8 & -5 & 1.09 & 10.53 & 47.9 & 20.4 & 21.6 & 20.5 & -1.0 & -1.0 \\
2 & 52.936641 & -27.790331 & -1.5 & -6 & 1.03 & 9.66 & 39.4 & 23.9 & 25.0 & 17.8 & 12.8 & 14.0 \\
3 & 52.950038 & -27.771467 & -1.8 & -6 & 0.88 & 9.14 & 51.3 & 26.1 & 27.3 & 24.0 & -1.0 & -1.0 \\
4 & 52.975983 & -27.732096 & -1.7 & -5 & 0.77 & 8.74 & 64.7 & 27.0 & 28.1 & 17.8 & 13.8 & 15.0 \\
5 & 53.001139 & -27.795137 & -1.9 & -5 & 0.76 & 6.23 & 45.9 & -1.0 & -1.0 & 22.8 & 10.3 \\
\hline
\end{tabular}

Note. The full table contains 73 columns of information for the 47 supplementary X-ray sources.

(This table is available in its entirety in machine-readable form.)
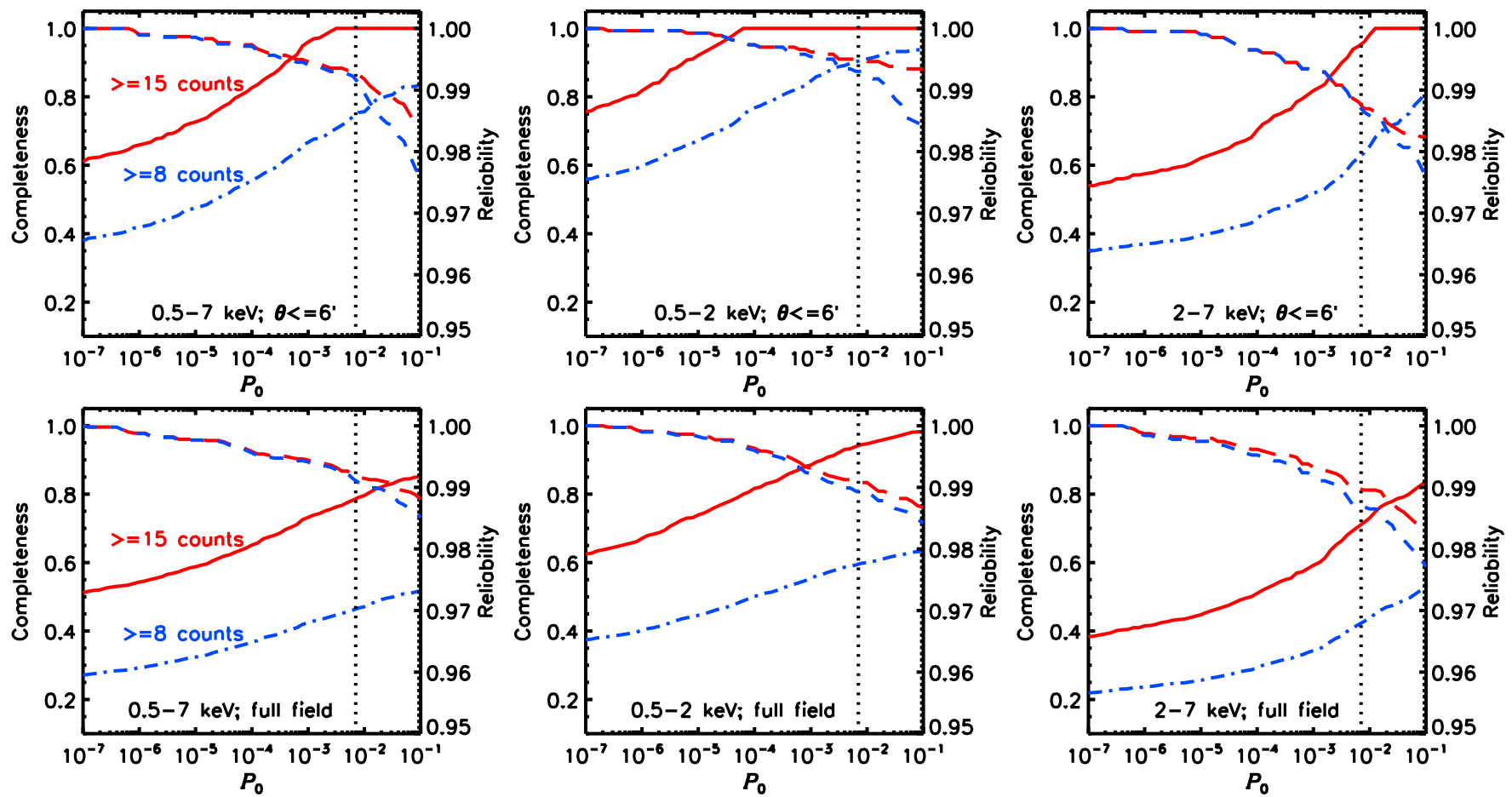

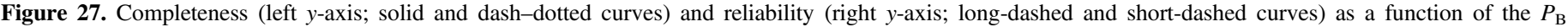

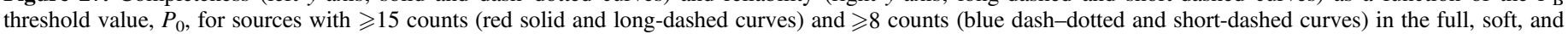

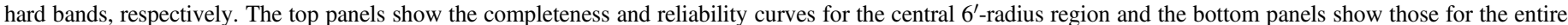
CDF-S field. The vertical dotted lines indicate our adopted main-catalog $P_{\mathrm{B}}$ threshold of 0.007 .

on the images at a false-positive probability threshold of $10^{-5}$ to obtain a candidate source list. We subsequently utilized AE to compute photometric properties and $P_{\mathrm{B}}$ values for these candidate-list sources.

By comparing the input sources and detected sources from the simulation, we can assess the completeness and reliability of our main catalog. For a given source-count limit, the completeness is defined as the fraction of the input sources detected above the count limit (the source recovery fraction), while the reliability is defined as 1 minus the ratio between the number of spurious sources (not in the input source list) above the count limit and the number of input sources above the count limit. As we further filtered the candidate sources with a $P_{\mathrm{B}}$ threshold cut during the second stage of our source-detection approach, the completeness and reliability also have a dependence on the adopted $P_{\mathrm{B}}$ threshold value, denoted as $P_{0}$. Figure 27 shows the completeness and reliability as a function of $P_{0}$ within the central $6^{\prime}$-radius region and over the entire CDF-S field, for sources with at least 15 and 8 counts in the full, soft, and hard bands, respectively; the minimum number of detected counts in the soft and hard bands for our main-catalog sources is $\approx 8$ (Table 2 ).

According to Figure 27, the detection completeness increases and the reliability decreases when the $P_{\mathrm{B}}$ threshold value is raised, as expected. For a larger source-count limit (15 versus 8 ), the completeness and reliability are both better, although the difference in reliability is negligible when the $P_{\mathrm{B}}$ threshold value is small (e.g., $P_{0}=10^{-5}$ ). Within the central $6^{\prime}$ radius region, our source detection achieves a better completeness overall compared to that in the entire CDF-S field. At our adopted main-catalog $P_{\mathrm{B}}$ threshold value of 0.007 , the completeness levels within the central 6 '-radius region are $100.0 \%$ and $74.7 \%$ (full band), $100.0 \%$ and $90.5 \%$ (soft band), and $95.3 \%$ and $62.8 \%$ (hard band) for sources with $\geqslant 15$ and 


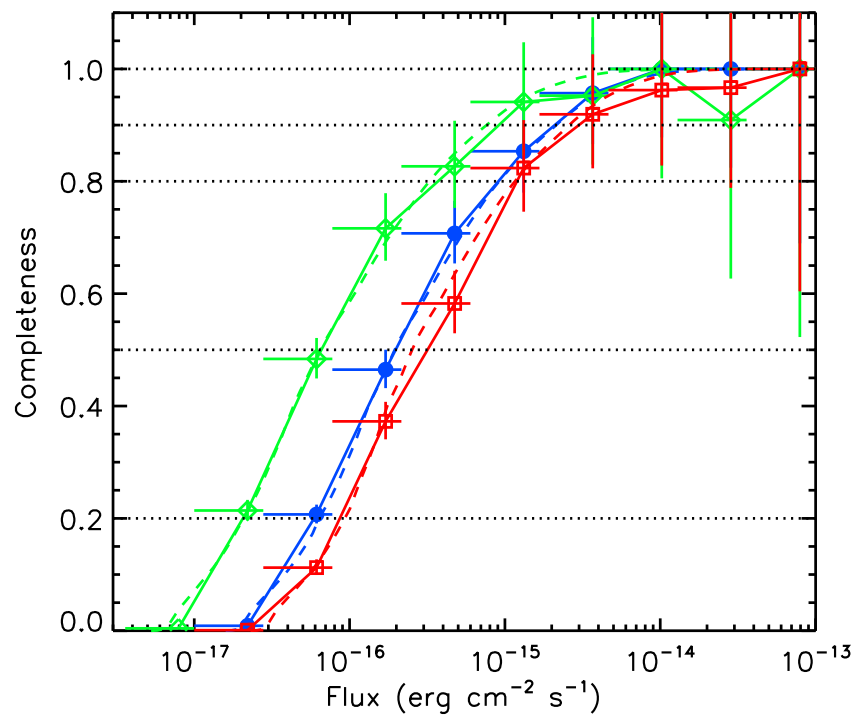

Figure 28. Completeness as a function of source flux in the full (blue filled circles), soft (green open diamonds), and hard (red open squares) bands, given our adopted main-catalog $P_{\mathrm{B}}$ threshold value of 0.007 . The solid lines link the corresponding completeness data points. Overlaid are the survey solid angle vs. sensitivity curves (dashed curves) normalized to the maximum solid angle (see Figure 30 below). The horizontal dotted lines denote five specific completeness levels $(100 \%, 90 \%, 80 \%, 50 \%$, and $20 \%)$.

$\geqslant 8$ counts, respectively. Across the entire CDF-S field, the completeness levels are $78.6 \%$ and $46.4 \%$ (full band), $94.2 \%$ and $59.5 \%$ (soft band), and $71.1 \%$ and $42.3 \%$ (hard band) for sources with $\geqslant 15$ and $\geqslant 8$ counts, respectively. At our adopted $P_{\mathrm{B}}$ threshold, the reliability levels range from $98.7 \%$ to $99.5 \%$ for all the cases (the central $6^{\prime}$-radius region or the entire field, $\geqslant 15$ or $\geqslant 8$ counts, in one of the three X-ray bands) in Figure 27 , suggesting that there are $\approx 7,6$, and 5 spurious detections with $\geqslant 15$ counts in the full, soft, and hard bands, and $\approx 7,7$, and 5 spurious detections with $\geqslant 8$ counts in the full, soft, and hard bands, respectively. The total number of spurious detections estimated from simulations is thus $\approx 19$. Most of these spurious sources should not have a multiwavelength counterpart; there are 16 such sources in our main catalog (Section 4.2).

At our adopted main-catalog $P_{\mathrm{B}}$ threshold value of 0.007 , the detection completeness as a function of source flux is presented in Figure 28. The completeness versus flux curves are consistent with the survey solid angle versus flux-limit curves (Figure 30 below) derived in Section 7 below, indicating that the completeness at a given flux is dominated by the CDF-S area fraction that is sensitive for detecting sources at this flux limit. In Table 6, we list the source fluxes at four specific completeness levels $(90 \%, 80 \%, 50 \%$, and $20 \%)$ in the full, soft, and hard bands, respectively.

\section{BACKGROUND AND SENSITIVITY ANALYSIS}

We created background and sensitivity maps following the procedure described in Section 7 of Xue et al. (2011). Briefly, we masked out the main and supplementary catalog sources from the raw images in the three X-ray bands, and we filled in the masked regions with random counts that are consistent with the local background level. The resulting background maps in the three bands were used to determine the detection sensitivity at each pixel location, which is the flux limit required for a source to be selected by our $\mathrm{AE} P_{\mathrm{B}}$ criterion. Given the
Table 6

Flux Limits and Completeness Levels

\begin{tabular}{lccc}
\hline $\begin{array}{l}\text { Completeness } \\
(\%)\end{array}$ & $\begin{array}{c}f_{0.5-7 \mathrm{keV}^{2}} \\
\left(\mathrm{erg} \mathrm{cm}^{-2} \mathrm{~s}^{-1}\right)\end{array}$ & $\begin{array}{c}f_{0.5-2 \mathrm{keV}^{-1}} \\
\left(\mathrm{erg} \mathrm{cm}^{-2} \mathrm{~s}^{-1}\right)\end{array}$ & $\begin{array}{c}f_{2-7 \mathrm{keV}} \\
\left(\mathrm{erg} \mathrm{cm}^{-2} \mathrm{~s}^{-1}\right)\end{array}$ \\
\hline 90 & $2.1 \times 10^{-15}$ & $9.1 \times 10^{-16}$ & $3.0 \times 10^{-15}$ \\
80 & $9.1 \times 10^{-16}$ & $3.7 \times 10^{-16}$ & $1.2 \times 10^{-15}$ \\
50 & $2.0 \times 10^{-16}$ & $6.6 \times 10^{-17}$ & $3.2 \times 10^{-16}$ \\
20 & $5.9 \times 10^{-17}$ & $2.1 \times 10^{-17}$ & $8.7 \times 10^{-17}$ \\
\hline
\end{tabular}

Table 7

Background Parameters

\begin{tabular}{|c|c|c|c|c|}
\hline \multirow{2}{*}{ Band (keV) } & \multicolumn{2}{|c|}{ Mean Background } & \multirow{2}{*}{$\begin{array}{c}\text { Total } \\
\text { Background } \\
\left(10^{5} \text { counts }\right) \\
\text { (4) }\end{array}$} & \multirow[b]{2}{*}{$\begin{array}{l}\text { Count Ratio } \\
\text { (background/ } \\
\text { source) } \\
\text { (5) }\end{array}$} \\
\hline & $\begin{array}{c}\text { (counts } \\
\text { pixel }^{-1} \text { ) } \\
\text { (2) }\end{array}$ & $\begin{array}{l}\text { (counts } \\
\mathrm{Ms}^{-1} \\
\text { pixel }^{-1} \text { ) } \\
\text { (3) }\end{array}$ & & \\
\hline Full (0.5-7.0) & 0.600 & 0.184 & 43.2 & 8.2 \\
\hline Soft $(0.5-2.0)$ & 0.173 & 0.055 & 12.5 & 4.2 \\
\hline Hard (2-7) & 0.427 & 0.127 & 30.7 & 13.8 \\
\hline
\end{tabular}

Note. Column 1: X-ray band. Column 2: mean number of background counts per pixel averaged across the background map (Section 7). Column 3: mean number of background counts per pixel (Column 2) divided by the mean effective exposure time averaged across the exposure map (Section 3.1); the mean effective exposure times are $3.26 \mathrm{Ms}, 3.18 \mathrm{Ms}$, and $3.37 \mathrm{Ms}$ for the full, soft, and hard bands, respectively. Column 4: total number of background counts measured from the background map. Column 5: ratio of the total number of background counts (Column 4) to the total number of source counts in the main and supplementary catalogs.

background level at each pixel location, we derived the minimum number of source counts required for a detection using Equation (1) and our adopted $P_{\mathrm{B}}$ threshold value of 0.007. Utilizing the exposure maps (Section 3.1) and assuming a simple power-law model with $\Gamma=1.4$, we converted the limiting count rates to limiting fluxes and produced sensitivity maps for the main catalog in the three X-ray bands.

The background properties for the $7 \mathrm{Ms}$ CDF-S are summarized in Table 7. The observed CDF-S background consists of several components, primarily the unresolved cosmic background, particle background, and instrumental background (e.g., Markevitch 2001; Markevitch et al. 2003); we do not attempt to separate these components for our analysis here. The mean numbers of background counts per pixel are small (0.17-0.60), indicating that many of the CDF-S pixels did not receive any X-ray photons over the $7 \mathrm{Ms}$ exposure. Indeed, in the full-band, soft-band, and hard-band raw images, $\approx 59 \%, 84 \%$, and $68 \%$ of the pixels have zero counts. The mean background count rate in the soft band $\left(0.055\right.$ counts $\mathrm{Ms}^{-1}$ pixel $\left.^{-1}\right)$ is $\approx 13 \%-17 \%$ smaller compared to those for the 2 Ms CDF-S (Luo et al. 2010) and 4 Ms CDF$\mathrm{S}$ (Xue et al. 2011), probably due to the decline of the ACIS effective area below $2 \mathrm{keV}$ (from build-up of contaminant on the ACIS optical blocking filters), ${ }^{48}$ and the increased sensitivity of the $7 \mathrm{Ms}$ CDF-S that resolves a larger fraction of the cosmic background, and/or variations of the particle and

\footnotetext{
48 For example, the ACIS-I soft-band effective area has dropped by $\approx 10 \%$ from Chandra Cycle 11 (proposal cycle for the $4 \mathrm{Ms}$ CDF-S) to Cycle 15 (proposal cycle for the $7 \mathrm{Ms}$ CDF-S). The $7 \mathrm{Ms}$ CDF-S observations span a broad range in time and not all the background components are affected by the decline of the ACIS effective area.
} 
instrumental background components over the past several years. The ratio between the total numbers of background and source counts in the soft band is approximately the same $(\approx 4.2)$ for the $2 \mathrm{Ms}, 4 \mathrm{Ms}$, and $7 \mathrm{Ms}$ CDF-S. In the full and hard bands, the mean background count rates are smaller $(\approx 25 \%)$ than those for the $2 \mathrm{Ms}$ and $4 \mathrm{Ms}$ CDF-S, mainly because we adopted a smaller upper energy bound of $7 \mathrm{keV}$ (instead of $8 \mathrm{keV}$ ) here. The ratio between the total numbers of background and source counts in the hard band $(\approx 14 ; 2-7 \mathrm{keV})$ is significantly lower than those $(\approx 20 ; 2-8 \mathrm{keV})$ for the $2 \mathrm{Ms}$ and 4 Ms CDF-S, confirming that it is advantageous to search for sources in the $2-7 \mathrm{keV}$ band instead of the $2-8 \mathrm{keV}$ band where the background has a larger contribution.

We are able to achieve unprecedented X-ray sensitivity in the 7 Ms CDF-S. The lowest estimated flux limits achievable are $\approx 1.5 \times 10^{-17}, 4.8 \times 10^{-18}$, and $2.1 \times 10^{-17} \mathrm{erg} \mathrm{cm}^{-2} \mathrm{~s}^{-1}$ in the full, soft, and hard bands, respectively, and the average achievable flux limits over the central $\approx 1 \operatorname{arcmin}^{2}$ region are $\approx 1.9 \times 10^{-17}, 6.4 \times 10^{-18}$, and $2.7 \times 10^{-17} \mathrm{erg} \mathrm{cm}^{-2} \mathrm{~s}^{-1}$. The lowest detected fluxes in the main catalog are actually around these limits, being $\approx 1.7 \times 10^{-17}, 7.7 \times 10^{-18}$, and $3.5 \times 10^{-17} \mathrm{erg} \mathrm{cm}^{-2} \mathrm{~s}^{-1}$ in the three bands. Compared to the average soft-band flux limit in the central $\approx 1 \operatorname{arcmin}^{2}$ region of the 4 Ms CDF-S $\left(9.1 \times 10^{-18} \mathrm{erg} \mathrm{cm}^{-2} \mathrm{~s}^{-1}\right.$; Xue et al. 2011), the $7 \mathrm{Ms}$ CDF-S sensitivity has been improved by a factor of 1.42. The full-band and hard-band sensitivities are not directly comparable, as the energy ranges are different. If we simply scale the $4 \mathrm{Ms}$ CDF-S $0.5-8.0 \mathrm{keV}$ and $2-8 \mathrm{keV}$ flux limits in the central region to the full $(0.5-7.0 \mathrm{keV})$ and hard $(2-7 \mathrm{keV})$ bands, assuming a $\Gamma=1.4$ power-law spectrum, the full-band sensitivity has been improved by a factor of $1.52\left(2.9 \times 10^{-17}\right.$ versus $1.9 \times 10^{-17} \mathrm{erg} \mathrm{cm}^{-2} \mathrm{~s}^{-1}$ ), and the hard-band sensitivity has been improved by a factor of $1.76\left(4.8 \times 10^{-17}\right.$ versus $2.7 \times 10^{-17} \mathrm{erg} \mathrm{cm}^{-2} \mathrm{~s}^{-1}$ ). The sensitivity improvement due to increased exposure in the full or hard band should be smaller than that in the soft band, because of the lower background level in the soft band (e.g., Table 7). The larger full- and hard-band improvement factors obtained for the 7 Ms CDF-S are due to the more sensitive $0.5-7.0 \mathrm{keV}$ and $2-7 \mathrm{keV}$ bands adopted for source detection where the background levels are lower compared to the $0.5-8.0 \mathrm{keV}$ and $2-8 \mathrm{keV}$ bands (see Footnote 16 of Xue et al. 2016 and our comparison of the background-tosource count ratios in the previous paragraph).

Beyond the small central region, the sensitivity drops with increasing off-axis angle. The full-band sensitivity map is displayed in Figure 29; flux limits in different ranges are shaded with different gray-scale levels. It is possible to detect sources with fluxes somewhat smaller than the sensitivity limits at their locations, due to the difference between the $\Gamma_{\text {eff }}$ values of the sources and the assumed value of 1.4 when computing the flux limits. There are 11 soft-band, 28 full-band, and 2 hard-band sources with fluxes $\approx 1 \%-13 \%$ below their corresponding flux limits. Given the spatial dependence of the sensitivity, we display in Figure 30 the survey solid angle versus flux limit in the full, soft, and hard bands. The flux limits for $\approx 50 \%$ of the CDF-S area are $\approx 2.0 \times 10^{-16}, 6.5 \times 10^{-17}$, and $2.5 \times 10^{-16} \mathrm{erg} \mathrm{cm}^{-2} \mathrm{~s}^{-1}$ in the full, soft, and hard bands, respectively, approximately an order of magnitude larger than those for the central $\approx 1 \operatorname{arcmin}^{2}$ region, and these values are consistent with the $50 \%$ completeness flux limits presented in Table 6.

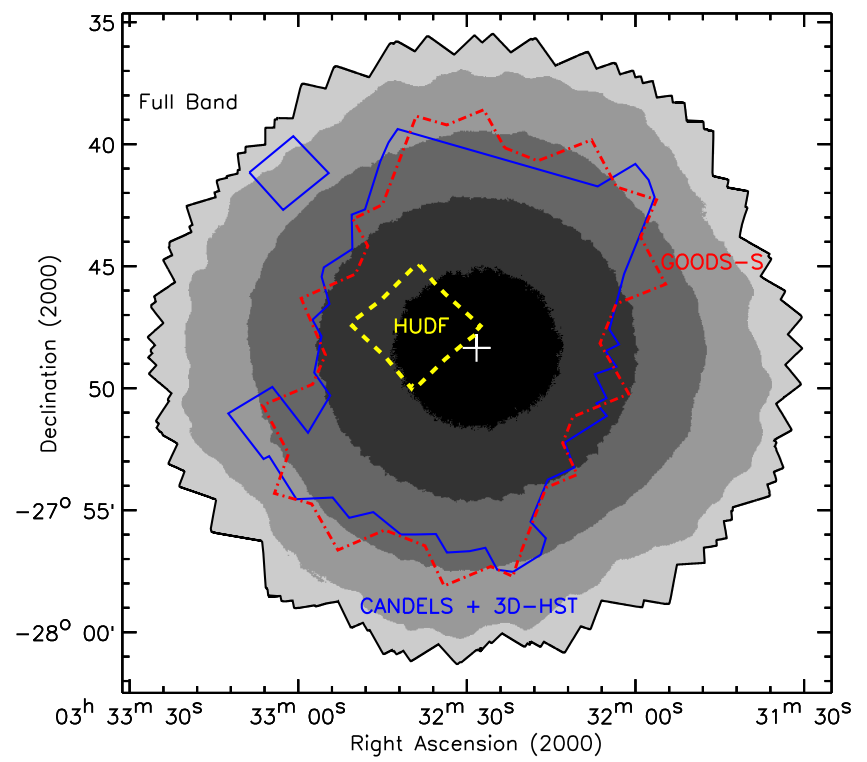

Figure 29. Full-band sensitivity map for the main source catalog. The grayscale levels, from black to light gray, represent regions with flux limits of $<3 \times 10^{-17}, \quad 3 \times 10^{-17}-8 \times 10^{-17}, 8 \times 10^{-17}-2 \times 10^{-16}, 2 \times 10^{-16}$ $10^{-15}$, and $>10^{-15} \mathrm{erg} \mathrm{cm}^{-2} \mathrm{~s}^{-1}$, respectively. The regions and the plus sign are the same as those in Figure 1.

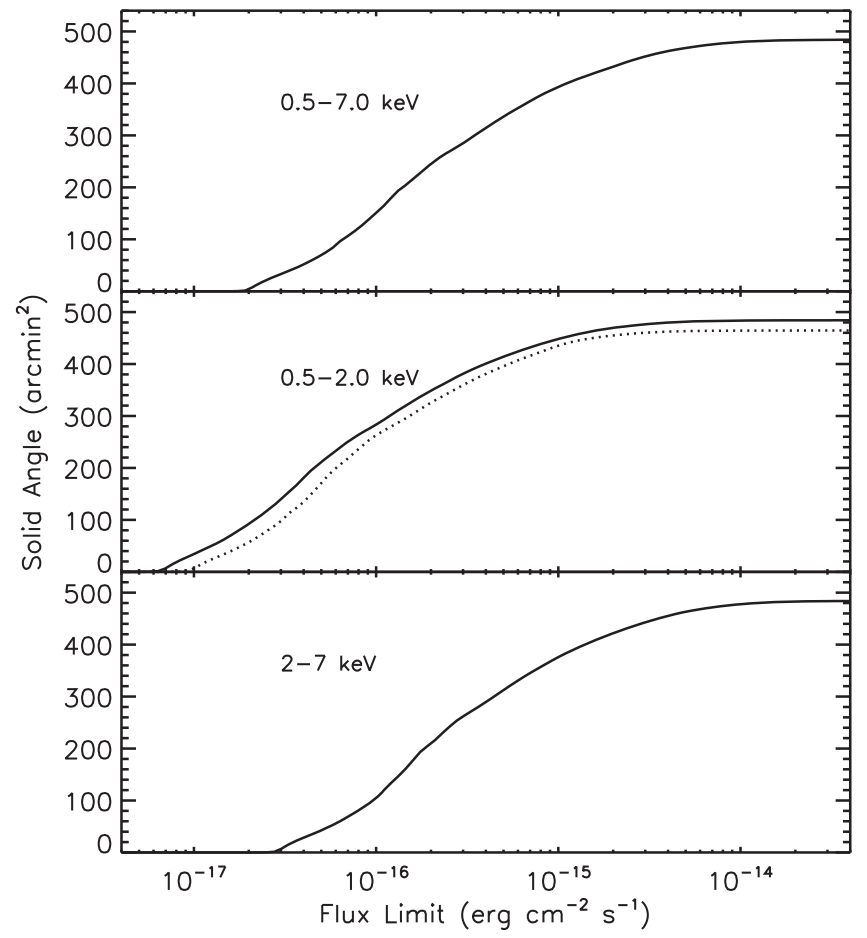

Figure 30. Survey solid angle as a function of flux limit in the full (top), soft (middle), and hard (bottom) bands. For comparison, the soft-band relation for the 4 Ms CDF-S (Xue et al. 2011) is displayed as the dotted curve in the middle panel (the full-band and hard-band results are not directly comparable due to the different choices of the energy bands). The $4 \mathrm{Ms}$ CDF-S curve can be approximately scaled to the $7 \mathrm{Ms}$ one by dividing the flux limits by a scaling factor of $\approx 1.38$.

We also compared the 7 Ms CDF-S soft-band sensitivityarea curve to the one for the 4 Ms CDF-S (Xue et al. 2011) in Figure 30 . The 4 Ms CDF-S curve can be approximately scaled to the $7 \mathrm{Ms}$ one by dividing the flux limits by a scaling factor of $\approx 1.38$, indicating that the 7 Ms CDF-S sensitivity has been 

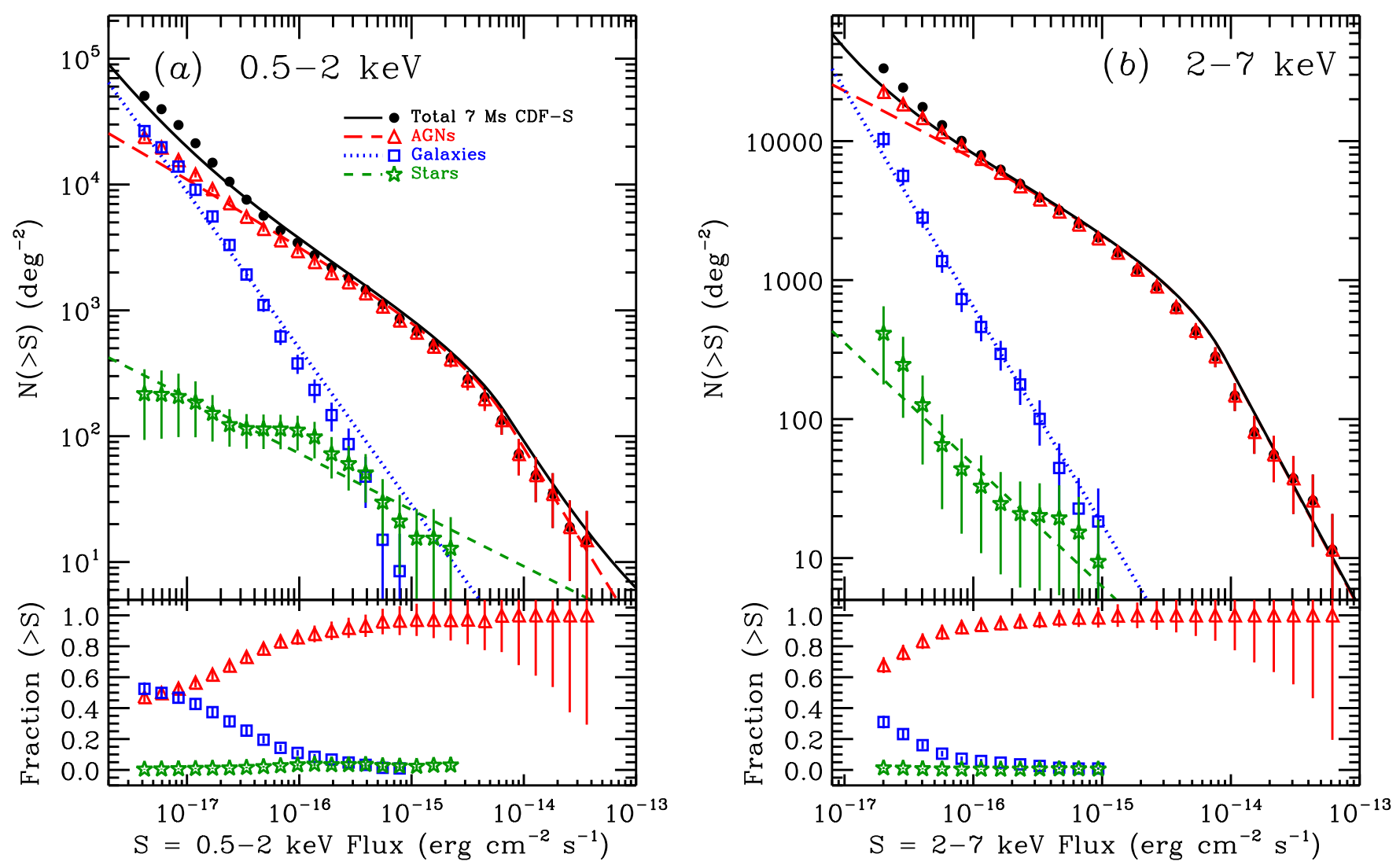

Figure 31. Cumulative number counts (number of sources brighter than a given flux) for the main source catalog (filled circles) in the (a) soft band and (b) hard band. The number counts are further broken down into AGNs (open red triangles), normal galaxies (open blue squares), and Galactic stars (open green stars) with their corresponding $1 \sigma$ errors displayed. The red long-dashed, blue dotted, and green short-dashed curves represent the number-count models based on the best-fit differential number-count models (Equation (3) and Table 8) for AGNs, galaxies, and stars, respectively, and the black curves show the number-count models for all X-ray sources (sum of the three components). The bottom panels show the fractional contributions from AGNs, galaxies, and stars to the total number counts. Galaxies provide significant contributions to the total number counts near the soft-band flux limit and they overtake AGNs at soft-band fluxes smaller than $\approx 6.0 \times 10^{-18}$ erg $\mathrm{cm}^{-2} \mathrm{~s}^{-1}$.

improved by a factor of $\approx 1.38$ on average. Such an improvement in sensitivity is expected given the factor of $\approx 1.75$ increase in exposure (see Section 7.3 of Xue et al. 2011) and the decrease of the ACIS-I soft-band effective area over the past years (see Footnote 48).

\section{NUMBER COUNTS FOR THE 7 MS CDF-S}

We computed the cumulative number of sources, $N(>S)$, brighter than a given intrinsic flux, $S$, for the soft and hard bands following the procedure described in Section 2 of Lehmer et al. (2012). Simulations based on our sourcedetection method were performed to obtain the source recovery functions, which account for detection incompleteness (see the Appendix of Lehmer et al. 2012 for details). A Bayesian approach was employed to obtain the flux-probability distributions, which account for Eddington bias (see Section 2.1 of Lehmer et al. 2012 for details). Unlike the creation of the sensitivity maps (Section 7), where simple count-rate-to-flux conversion factors were used, we consider here the conversion to be probabilistic. A Bayesian prior based on the differential number-count models $\left(d N /\left.d S\right|_{\text {model }}\right)$ is utilized in determining the flux-probability distributions, and following Lehmer et al. (2012), we adopted double or single power-law forms for the number-count models of AGNs, normal galaxies, and Galactic stars:

$$
\begin{aligned}
& \frac{d N}{d S}^{\mathrm{AGN}}=\left\{\begin{array}{cc}
K^{\mathrm{AGN}}\left(S / S_{\mathrm{ref}}\right)^{-\beta_{1}^{\mathrm{AGN}}} & \left(S \leqslant f_{\mathrm{b}}^{\mathrm{AGN}}\right) \\
K^{\mathrm{AGN}}\left(f_{\mathrm{b}} / S_{\mathrm{ref}}\right)^{\beta_{2}^{\mathrm{AGN}}-\beta_{1}^{\mathrm{AGN}}}\left(S / S_{\mathrm{ref}}\right)^{-\beta_{2}^{\mathrm{AGN}}} & \left(S>f_{\mathrm{b}}^{\mathrm{AGN}}\right)
\end{array}\right. \\
& {\frac{d N^{\mathrm{gal}}}{d S}}^{\mathrm{g}}=K^{\mathrm{gal}}\left(S / S_{\mathrm{ref}}\right)^{-\beta^{\mathrm{gal}}} \\
& \frac{d N^{\text {star }}}{d S}=K^{\text {star }}\left(S / S_{\text {ref }}\right)^{-\beta^{\text {star }}},
\end{aligned}
$$

where $S_{\text {ref }}=10^{-14} \mathrm{erg} \mathrm{cm}^{-2} \mathrm{~s}^{-1}$. As shown later in Figure 31, these power-law models provide acceptable descriptions of the overall shapes of the cumulative number counts.

To derive the best-fit model parameters in Equation (3), a maximum-likelihood technique was utilized to maximize the total likelihood of obtaining the main source catalog and its source-count distribution (see Section 2.2 of Lehmer et al. 2012 for details). The best-fit parameters and the corresponding $1 \sigma$ uncertainties are listed in Table 8. After determining the Bayesian prior for the flux-probability distributions, the cumulative number counts for the soft and hard bands were computed based on the main-catalog sources, broken down into AGNs, galaxies, and stars. In Figure 31, we present the cumulative number counts for the three source populations as well as all the X-ray sources in the soft and hard bands, respectively. The number-count models derived from the best- 
Table 8

Differential Number-count Prior Best-fit Model Parameters

\begin{tabular}{|c|c|c|c|c|c|c|c|c|}
\hline \multirow{2}{*}{ Band (keV) } & \multicolumn{4}{|c|}{ AGNs } & \multicolumn{2}{|c|}{ Galaxies } & \multicolumn{2}{|c|}{ Stars } \\
\hline & $\begin{array}{c}K_{14}^{\mathrm{AGN}} \\
(2)\end{array}$ & $\begin{array}{c}\beta_{1}^{\mathrm{AGN}} \\
(3)\end{array}$ & $\begin{array}{c}\beta_{2}^{\mathrm{AGN}} \\
\text { (4) }\end{array}$ & $\begin{array}{c}f_{\text {break }}^{\text {AGN }} \\
(5)\end{array}$ & $\begin{array}{c}K_{14}^{\mathrm{gal}} \\
(6)\end{array}$ & $\begin{array}{l}\beta^{\text {gal }} \\
(7)\end{array}$ & $\begin{array}{c}K_{14}^{\text {star }} \\
(8)\end{array}$ & $\begin{array}{c}\beta^{\text {star }} \\
(9)\end{array}$ \\
\hline Soft (0.5-2.0) & $161.96 \pm 7.10$ & $1.52 \pm 0.03$ & $2.45 \pm 0.30$ & $7.1_{-2.4}^{+2.5}$ & $2.01 \pm 0.10$ & $2.24 \pm 0.06$ & $4.14 \pm 0.30$ & $1.45_{-0.12}^{+0.13}$ \\
\hline Hard (2-7) & $453.70 \pm 20.61$ & $1.46 \pm 0.03$ & $2.72 \pm 0.24$ & $8.9 \pm 1.7$ & $0.75 \pm 0.09$ & $2.56 \pm 0.15$ & $0.73 \pm 0.13$ & $1.88_{-0.35}^{+0.36}$ \\
\hline
\end{tabular}

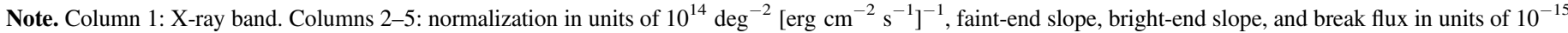

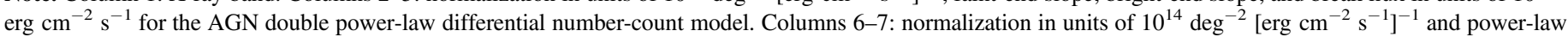

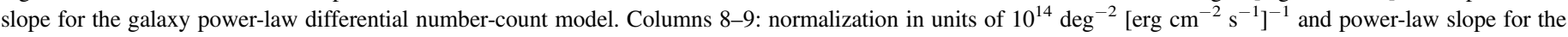
star power-law differential number-count model.

fit differential models (Equation (3) and Table 8) are also displayed, showing general agreement with the data.

Our number-count calculations implement a probabilistic approach, in which we calculated, at each location in our images, the probability of detection as a function of flux and photon index (see Section 2.1 of Lehmer et al. 2012). As such, there are sufficiently large areas of the survey in which sources with fluxes below the sensitivity limits quoted in Section 7 could be detected. The flux limits for $\approx 10 \operatorname{arcmin}^{2}$ effective areas derived from this approach are $4.2 \times 10^{-18}$ erg cm $\mathrm{cm}^{-2} \mathrm{~s}^{-1}$ in the soft band and $2.0 \times 10^{-17} \mathrm{erg} \mathrm{cm}^{-2} \mathrm{~s}^{-1}$ in the hard band. Unlike the AGN number counts, where a break flux around $10^{-15} \mathrm{erg} \mathrm{cm} \mathrm{cm}^{-2} \mathrm{~s}^{-1}$ is evident, the galaxy number counts follow a single power-law form and continue to rise down to the lowest fluxes observed. At the faint end, the galaxy number counts rise more sharply with power-law slopes of $\approx 1.2-1.6\left(\beta^{\mathrm{gal}}-1\right)$ than the AGN number counts with faint-end power-law slopes of $\approx 0.5$.

The bottom panels of Figure 31 show the fractional contributions from AGNs, galaxies, and stars to the total number counts. The contribution from galaxies increases toward lower fluxes, especially in the soft band, which is more sensitive than the hard band. Similar trends have been observed in previous studies of number counts in deep X-ray surveys (e.g., Bauer et al. 2004; Ranalli et al. 2005; Lehmer et al. 2012), and it has been suggested that the galaxy number counts will overtake the AGN number counts just below the 4 Ms CDF-S soft-band flux limit (e.g., Lehmer et al. 2012). Indeed, thanks to the unprecedented sensitivity of the $7 \mathrm{Ms}$ CDF-S, we observe for the first time that the galaxy number counts exceed the AGN number counts at soft-band fluxes smaller than $\approx 6.0 \times 10^{-18} \mathrm{erg} \mathrm{cm}^{-2} \mathrm{~s}^{-1}$. At the soft-band flux limit $\left(4.2 \times 10^{-18} \mathrm{erg} \mathrm{cm}^{-2} \mathrm{~s}^{-1}\right)$, the AGN density is $\approx 23,900$ $\mathrm{deg}^{-2}, 47 \% \pm 4 \%$ of the total X-ray source density $(\approx 50,500$ $\mathrm{deg}^{-2}$, while the galaxy density reaches $\approx 26,600 \mathrm{deg}^{-2}$, $52 \% \pm 5 \%$ of the total, indicating that normal galaxies start to dominate the X-ray source population at the faintest flux levels. At this flux limit, the entire sky contains $\approx 1.0$ billion AGNs and $\approx 1.1$ billion galaxies. We caution that due to cosmic variance, the CDF-S field might not be a representative patch of the entire sky, and small field-to-field variations of the X-ray source distribution have been observed between the CDF-S and other survey fields (e.g., Bauer et al. 2004; Luo et al. 2008).

Among the main-catalog sources, there are 264 X-ray galaxies (excluding AGNs) with $K_{s} \leqslant 22$, which constitute $4.0 \% \pm 0.2 \%$ of the $6651 K_{s} \leqslant 22$ TENIS sources within the $7 \mathrm{Ms}$ CDF-S field of view. The fraction of X-ray-detected galaxies increases toward better X-ray sensitivity; within the innermost $2^{\prime}$-radius region, there are $31 K_{s} \leqslant 22 \mathrm{X}$-ray galaxies, $24 \% \pm 4 \%$ of the $129 K_{s} \leqslant 22$ TENIS sources in this region. With the number-count results, we further investigated the fraction of galaxies that are detectable in deep X-ray surveys as a function of the X-ray limiting flux, accounting for detection incompleteness and Eddington bias. At the soft-band flux limit $\left(4.2 \times 10^{-18} \mathrm{erg} \mathrm{cm}^{-2} \mathrm{~s}^{-1}\right)$, the density of $K_{s} \leqslant 22 \mathrm{X}$-ray galaxies is $\approx 22300 \mathrm{deg}^{-2}$ (derived similarly to those in Figure 31 ). The TENIS catalog is complete at $K_{s} \leqslant 22$, and we verified its completeness by comparing its $K_{s}$-band galaxy number counts to previous number-count results (e.g., Saracco et al. 2001). Excluding 295 stars from the 6651 TENIS sources identified from spectroscopic catalogs, the $K_{s} \leqslant 22$ galaxy density is $\approx 47300 \mathrm{deg}^{-2}$. Therefore, the X-ray detected $K_{s} \leqslant 22$ galaxies account for $\approx 47 \%$ of all $K_{s} \leqslant 22$ galaxies at the soft-band flux limit. The fraction of $K_{s} \leqslant 22$ galaxies detected in the $\mathrm{X}$-rays decreases rapidly with increasing soft-band limiting flux, being $\approx 20 \%$ at a limiting flux of $10^{-17} \mathrm{erg} \mathrm{cm}^{-2} \mathrm{~s}^{-1}$ and $\approx 2 \%$ at a limiting flux of $5 \times 10^{-17} \mathrm{erg} \mathrm{cm}^{-2} \mathrm{~s}^{-1}$.

Using the number-count estimates derived above, we integrated the X-ray source fluxes to obtain the fraction of the cosmic X-ray background (CXRB) that is resolved into point sources at the $7 \mathrm{Ms} \mathrm{CDF-S} \mathrm{flux} \mathrm{limit.} \mathrm{Our} \mathrm{analysis} \mathrm{followed} \mathrm{the} \mathrm{same} \mathrm{procedure}$ described in Section 3.4 of Lehmer et al. (2012). We adopted total CXRB intensities of $(8.15 \pm 0.58) \times 10^{-12} \mathrm{erg} \mathrm{cm}^{-2} \mathrm{~s}^{-1}$ and $(1.49 \pm 0.20) \times 10^{-11} \mathrm{erg} \mathrm{cm}^{-2} \mathrm{~s}^{-1}$ in the soft and hard bands, respectively (Hickox \& Markevitch 2006; Kim et al. 2007b). The resolved CXRB fractions, after including the contributions from the relatively rare X-ray bright-source population (Kim et al. 2007b), are $80.9 \% \pm 4.4 \%$ and $92.7 \% \pm 13.3 \%$ in the soft and hard bands, respectively. For comparison, the resolved CXRB fractions at the 4 Ms CDF-S flux limit (Lehmer et al. 2012) are $75.7 \% \pm 4.3 \%$ and $82.4 \% \pm 13.0 \%$ in the soft band and the 2-8 keV band, respectively.

\section{SUMMARY}

We have presented X-ray source catalogs for the deepest Chandra X-ray survey, the $7 \mathrm{Ms}$ CDF-S. The main points from this work are summarized below.

1. The entire $7 \mathrm{Ms}$ CDF-S consists of 102 individual observations covering a total area of $484.2 \mathrm{arcmin}^{2}$. The cleaned net exposure time is $6.727 \mathrm{Ms}$. See Section 2.

2. The main Chandra source catalog contains $1008 \mathrm{X}$-ray sources that were detected by WAVDETECT with a falsepositive probability threshold of $1 \times 10^{-5}$ and filtered by 
AE with a binomial no-source probability $\left(P_{\mathrm{B}}\right)$ threshold of 0.007 . These sources were detected in up to three X-ray bands: $0.5-7.0 \mathrm{keV}$ (full band), 0.5-2.0 keV (soft band), and 2-7 keV (hard band). See Section 3.2.

3. The supplementary Chandra source catalog contains 47 $\mathrm{X}$-ray sources that were detected by WAVDETECT with a false-positive probability threshold of $1 \times 10^{-5}$, have $0.007 \leqslant P_{\mathrm{B}}<0.1$, and are matched to bright $\left(K_{s} \leqslant 23\right)$ NIR counterparts. See Section 3.2.

4. The absolute astrometry of the $7 \mathrm{Ms}$ CDF-S was registered to the TENIS NIR astrometric frame. The $\mathrm{X}$-ray source positions were determined based on their centroid or matched-filter positions. For the main-catalog sources, the $1 \sigma$ positional uncertainties range from $0 . " 11$ to $1 . " 28$, with a median value of $0 . " 47$. See Sections 2.2, 3.2 , and 4.1.

5. We identified optical/NIR/IR counterparts for 992 (98.4\%) of the main-catalog sources, with an average false-match rate of $\approx 1.6 \%$. Basic counterpart information in the optical through radio catalogs for the 992 sources are provided. Most of the 16 sources without multiwavelength counterparts are likely spurious detections. See Section 4.2.

6. We collected redshifts from public catalogs for 986 maincatalog sources, including 653 spectroscopic redshifts and 333 photometric redshifts. The photometric redshifts are of high quality in general. The median redshift for these X-ray sources is $1.12 \pm 0.05$ with an interquartile range of 0.67-1.95. See Section 4.3.

7. For the main catalog, the median numbers of source counts in the full, soft, and hard bands are 98.9, 47.7, and 94.6, respectively. There are 456 sources with $>100$ full-band counts, and 90 with $>1000$ full-band counts. The median $\mathrm{X}$-ray fluxes in the full, soft, and hard bands are $3.1 \times 10^{-16}, 6.5 \times 10^{-17}$, and $5.7 \times 10^{-16} \mathrm{erg} \mathrm{cm}^{-2} \mathrm{~s}^{-1}$, respectively. There are 613 sources with absorptioncorrected intrinsic $0.5-7.0 \mathrm{keV}$ luminosities above $10^{42} \mathrm{erg} \mathrm{s}^{-1}$ and 108 sources above $10^{44} \mathrm{erg} \mathrm{s}^{-1}$. See Section 4.4.

8. We identified 711 AGNs (71\%) from the main catalog based on their X-ray and multiwavelength properties. Besides 12 Galactic stars, the remaining 285 sources are likely normal galaxies. See Section 4.5.

9. We detected 291 new X-ray sources in the 7 Ms CDF-S main catalog compared to the $4 \mathrm{Ms}$ CDF-S catalogs, three of which were outside the footprint of the $4 \mathrm{Ms}$ CDF-S. A smaller fraction of the new sources are classified as AGNs (63\%) compared to that for the entire catalog (71\%). The median redshifts for the new AGNs and galaxies are comparable to those for the entire AGN and galaxy samples. The new AGNs have a lower median flux and median luminosity than the old AGNs. The new galaxies have a slightly lower median flux than the old galaxies, while their median luminosities are comparable. See Section 4.6.

10. Simulations suggest that our main catalog is highly reliable with $\approx 7,7$, and 5 spurious detections in the full, soft, and hard bands, respectively. The completeness levels within the central $6^{\prime}$-radius region are $74.7 \%$, $90.5 \%$, and $62.8 \%$ for sources with $\geqslant 8$ counts in the full, soft, and hard bands, respectively, and they are higher for sources with larger numbers of counts. See Section 6.
11. The mean numbers of background counts per pixel are still small (0.17-0.60 in the three bands) in the $7 \mathrm{Ms}$ Chandra exposure; most of the pixels have zero counts. The low background level and deep exposure result in unprecedented X-ray sensitivity for the $7 \mathrm{Ms}$ CDF-S. The average flux limits over the central $\approx 1 \operatorname{arcmin}^{2}$ region reach $\approx 1.9 \times 10^{-17}, 6.4 \times 10^{-18}$, and $2.7 \times 10^{-17}$ erg $\mathrm{cm}^{-2} \mathrm{~s}^{-1}$ in the full, soft, and hard bands, respectively, and the flux limits for $\approx 50 \%$ of the CDF-S area are $\approx 2.0 \times 10^{-16}, 6.5 \times 10^{-17}$, and $2.5 \times 10^{-16}$ erg $\mathrm{cm}^{-2} \mathrm{~s}^{-1}$. Compared to the average soft-band flux limit in the central $\approx 1 \operatorname{arcmin}^{2}$ region of the $4 \mathrm{Ms} C D F-$ $\mathrm{S}$, the $7 \mathrm{Ms}$ CDF-S sensitivity has been improved by a factor of 1.42. See Section 7.

12. We computed cumulative number counts down to the softband flux limit of $4.2 \times 10^{-18} \mathrm{erg} \mathrm{cm}^{-2} \mathrm{~s}^{-1}$ and hard-band flux limit of $2.0 \times 10^{-17} \mathrm{erg} \mathrm{cm}^{-2} \mathrm{~s}^{-1}$. The number counts are broken down into AGNs, normal galaxies, and Galactic stars. After correcting for detection incompleteness and Eddington bias, the AGN density is $\approx 23,900 \mathrm{deg}^{-2}$ ( $47 \% \pm 4 \%$ of the total source density) and the galaxy density is $\approx 26,600 \mathrm{deg}^{-2}(52 \% \pm 5 \%$ of the total $)$ at the soft-band flux limit. We observe, for the first time, that normal galaxies start to dominate the X-ray source population at the faintest flux levels $\left(\lesssim 6.0 \times 10^{-18}\right.$ erg cm $\mathrm{cm}^{-2} \mathrm{~s}^{-1}$ ). The resolved CXRB fractions computed using the number-count estimates are $80.9 \% \pm 4.4 \%$ and $92.7 \% \pm 13.3 \%$ in the soft and hard bands, respectively. See Section 8.

The $7 \mathrm{Ms}$ CDF-S will serve as a multi-decade Chandra legacy for advancing deep-survey science projects, owing to its unique combination of great depth and high angular resolution. Detailed science results for the $7 \mathrm{Ms}$ CDF-S are presented in additional papers (e.g., Lehmer et al. 2016; Vito et al. 2016; Yang et al. 2016).

We acknowledge financial support from the National Thousand Young Talents program of China (B.L., Y.Q.X.), National Natural Science Foundation of China grant 11673010 (B.L.), Ministry of Science and Technology of China grant 2016YFA0400702 (B.L.), CXC grant GO4-15130A (B.L., W.N.B., F.V., G.Y.), 973 Program $2015 C B 857004$ (Y.Q.X., M.S.) and 2015CB857005 (J.X.W.), NSFC-11473026 (Y.Q.X., M.S.), NSFC-11421303 (Y.Q.X., M.S., J.X.W.), Strategic Priority Research Program of CAS grant XDB09000000 (Y.Q.X., M.S., J.X.W.), Fundamental Research Funds for the Central Universities (Y.Q.X., M.S.), CAS Frontier Science Key Research Program QYZDJ-SSW-SLH006 (Y.Q.X., M.S., J.X.W.), STFC grant ST/L00075X/1 (D.M.A., I.S.), CONICYT-Chile grants Basal-CATA PFB-06/2007 (F.E.B.), FONDECYT Regular 1141218 (F.E.B.), "EMBIGGEN" Anillo ACT1101 (F.E.B.), the Ministry of Economy, Development, and Tourism's Millennium Science Initiative through grant IC120009, awarded to the Millennium Institute of Astrophysics, MAS (F.E.B.), ERC Advanced grant DUSTYGAL 321334 (I.S.), and the Royal Society/Wolfson Merit Award (I.S.).

\section{REFERENCES}

Alexander, D. M., Bauer, F. E., Brandt, W. N., et al. 2003, AJ, 126, 539 Allen, S. W., Evrard, A. E., \& Mantz, A. B. 2011, ARA\&A, 49, 409 
Arnaud, K. A. 1996, in ASP Conf. Ser. 101, Astronomical Data Analysis Software and Systems V, ed. G. H. Jacoby \& J. Barnes (San Francisco, CA: ASP), 17

Ashby, M. L. N., Willner, S. P., Fazio, G. G., et al. 2013, ApJ, 769, 80

Baganoff, F. K., Maeda, Y., Morris, M., et al. 2003, ApJ, 591, 891

Balestra, I., Mainieri, V., Popesso, P., et al. 2010, A\&A, 512, A12

Barcons, X., Nandra, K., Barret, D., et al. 2015, JPhCS, 610, 012008

Bauer, F. E., Alexander, D. M., Brandt, W. N., et al. 2004, AJ, 128, 2048

Beckwith, S. V. W., Stiavelli, M., Koekemoer, A. M., et al. 2006, AJ, 132, 1729

Blain, A. W. 1996, MNRAS, 283, 1340

Brandt, W. N., \& Alexander, D. M. 2015, A\&ARv, 23, 1

Broos, P. S., Townsley, L. K., Feigelson, E. D., et al. 2010, ApJ, 714, 1582

Broos, P. S., Townsley, L. K., Feigelson, E. D., et al. 2011, ApJS, 194, 2

Broos, P. S., et al. 2000, User's Guide for the TARA Package (Univ Park: Pennsylvania Sate Univ.) http://www.astro.psu.edu/xray/docs/TARA/

Caldwell, J. A. R., McIntosh, D. H., Rix, H.-W., et al. 2008, ApJS, 174, 136

Cappelluti, N., Brusa, M., Hasinger, G., et al. 2009, A\&A, 497, 635

Cappelluti, N., Comastri, A., Fontana, A., et al. 2016, ApJ, 823, 95

Cappelluti, N., Hasinger, G., Brusa, M., et al. 2007, ApJS, 172, 341

Casey, C. M., Chapman, S. C., Smail, I., et al. 2011, MNRAS, 411, 2739

Chapman, S. C., Smail, I., Ivison, R. J., \& Blain, A. W. 2002, MNRAS, 335, L17

Civano, F., Marchesi, S., Comastri, A., et al. 2016, ApJ, 819, 62

Colless, M., Peterson, B. A., Jackson, C., et al. 2003, arXiv:astro-ph/0306581

Cooper, M. C., Yan, R., Dickinson, M., et al. 2012, MNRAS, 425, 2116

Danielson, A. L. R., Swinbank, A. M., Smail, I., et al. 2017, ApJ, submitted

Dehghan, S., \& Johnston-Hollitt, M. 2014, AJ, 147, 52

Del Moro, A., Mullaney, J. R., Alexander, D. M., et al. 2014, ApJ, 786, 16

Doherty, M., Bunker, A. J., Ellis, R. S., \& McCarthy, P. J. 2005, MNRAS, 361,525

Feruglio, C., Daddi, E., Fiore, F., et al. 2011, ApJ, 729, L4

Finoguenov, A., Tanaka, M., Cooper, M., et al. 2015, A\&A, 576, A130

Fiore, F., Puccetti, S., Grazian, A., et al. 2012, A\&A, 537, A16

Freeman, P. E., Kashyap, V., Rosner, R., \& Lamb, D. Q. 2002, ApJS, 138, 185

Gaia Collaboration, Brown, A. G. A., Vallenari, A., et al. 2016, A\&A, 595, A2

Garmire, G. P., Bautz, M. W., Ford, P. G., Nousek, J. A., \& Ricker, G. R., Jr. 2003, Proc. SPIE, 4851, 28

Gehrels, N. 1986, ApJ, 303, 336

Gendreau, K. C., Mushotzky, R., Fabian, A. C., et al. 1995, PASJ, 47, L5

Giacconi, R., Zirm, A., Wang, J., et al. 2002, ApJS, 139, 369

Giallongo, E., Grazian, A., Fiore, F., et al. 2015, A\&A, 578, A83

Giavalisco, M., Ferguson, H. C., Koekemoer, A. M., et al. 2004, ApJL, 600, L93

Gilli, R., Cimatti, A., Daddi, E., et al. 2003, ApJ, 592, 721

Grazian, A., Fontana, A., de Santis, C., et al. 2006, A\&A, 449, 951

Grogin, N. A., Kocevski, D. D., Faber, S. M., et al. 2011, ApJS, 197, 35

Hasinger, G., Burg, R., Giacconi, R., et al. 1998, A\&A, 329, 482

Hickox, R. C., \& Markevitch, M. 2006, ApJ, 645, 95

Hornschemeier, A. E., Brandt, W. N., Garmire, G. P., et al. 2001, ApJ, 554,742

Hsieh, B.-C., Wang, W.-H., Hsieh, C.-C., et al. 2012, ApJS, 203, 23

Hsu, L.-T., Salvato, M., Nandra, K., et al. 2014, ApJ, 796, 60

Iwasawa, K., Gilli, R., Vignali, C., et al. 2012, A\&A, 546, A84

Jansen, F., Lumb, D., Altieri, B., et al. 2001, A\&A, 365, L1

Kim, M., Kim, D.-W., Wilkes, B. J., et al. 2007a, ApJS, 169, 401

Kim, M., Wilkes, B. J., Kim, D.-W., et al. 2007b, ApJ, 659, 29

Koekemoer, A. M., Faber, S. M., Ferguson, H. C., et al. 2011, ApJS, 197, 36

Kraft, R. P., Burrows, D. N., \& Nousek, J. A. 1991, ApJ, 374, 344

Kriek, M., van Dokkum, P. G., Franx, M., et al. 2008, ApJ, 677, 219

Kurk, J., Cimatti, A., Daddi, E., et al. 2013, A\&A, 549, A63

Le Fèvre, O., Cassata, P., Cucciati, O., et al. 2013, A\&A, 559, A14

Lehmer, B. D., Alexander, D. M., Chapman, S. C., et al. 2009, MNRAS, 400, 299
Lehmer, B. D., Basu-Zych, A. R., Mineo, S., et al. 2016, ApJ, 825, 7

Lehmer, B. D., Brandt, W. N., Alexander, D. M., et al. 2005, ApJS, 161, 21

Lehmer, B. D., Brandt, W. N., Hornschemeier, A. E., et al. 2006, AJ, 131, 2394

Lehmer, B. D., Xue, Y. Q., Brandt, W. N., et al. 2012, ApJ, 752, 46

Luo, B., Bauer, F. E., Brandt, W. N., et al. 2008, ApJS, 179, 19

Luo, B., Brandt, N., \& Bauer, F. 2014a, ATel, 6541

Luo, B., Brandt, W. N., \& Bauer, F. 2014b, ATel, 6625

Luo, B., Brandt, W. N., Xue, Y. Q., et al. 2010, ApJS, 187, 560

Lyons, L. 1991, Data Analysis for Physical Science Students (Cambridge: Cambridge Univ. Press)

Mao, M. Y., Sharp, R., Norris, R. P., et al. 2012, MNRAS, 426, 3334

Markevitch, M. 2001, CXC Memo (Cambridge: CXC) http://cxc.harvard.edu/ cal/calreview/mm_calrev_bg.ps

Markevitch, M., Bautz, M. W., Biller, B., et al. 2003, ApJ, 583, 70

Marshall, F. E., Boldt, E. A., Holt, S. S., et al. 1980, ApJ, 235, 4

Mignoli, M., Cimatti, A., Zamorani, G., et al. 2005, A\&A, 437, 883

Miller, N. A., Bonzini, M., Fomalont, E. B., et al. 2013, ApJS, 205, 13

Mineo, S., Gilfanov, M., Lehmer, B. D., Morrison, G. E., \& Sunyaev, R. 2014, MNRAS, 437, 1698

Miniutti, G., Brandt, W. N., Schneider, D. P., et al. 2012, MNRAS, 425, 1718

Morris, A. M., Kocevski, D. D., Trump, J. R., et al. 2015, AJ, 149, 178

Oke, J. B., \& Gunn, J. E. 1983, ApJ, 266, 713

Park, T., Kashyap, V. L., Siemiginowska, A., et al. 2006, ApJ, 652, 610

Planck Collaboration, Ade, P. A. R., Aghanim, N., et al. 2016, A\&A, 594, A13

Puccetti, S., Vignali, C., Cappelluti, N., et al. 2009, ApJS, 185, 586

Rafferty, D. A., Brandt, W. N., Alexander, D. M., et al. 2011, ApJ, 742, 3

Ranalli, P., Comastri, A., \& Setti, G. 2005, A\&A, 440, 23

Ranalli, P., Comastri, A., Vignali, C., et al. 2013, A\&A, 555, A42

Rauch, M., Becker, G. D., Haehnelt, M. G., Carswell, R. F., \& Gauthier, J.-. R. 2013, MNRAS, 431, L68

Ravikumar, C. D., Puech, M., Flores, H., et al. 2007, A\&A, 465, 1099

Rosati, P., Tozzi, P., Giacconi, R., et al. 2002, ApJ, 566, 667

Santini, P., Ferguson, H. C., Fontana, A., et al. 2015, ApJ, 801, 97

Saracco, P., Giallongo, E., Cristiani, S., et al. 2001, A\&A, 375, 1

Silverman, J. D., Mainieri, V., Salvato, M., et al. 2010, ApJS, 191, 124

Simpson, J. M., Smail, I., Swinbank, A. M., et al. 2015, ApJ, 799, 81

Skelton, R. E., Whitaker, K. E., Momcheva, I. G., et al. 2014, ApJS, 214, 24

Stark, A. A., Gammie, C. F., Wilson, R. W., et al. 1992, ApJS, 79, 77

Straatman, C. M. S., Spitler, L. R., Quadri, R. F., et al. 2016, ApJ, 830, 51

Szokoly, G. P., Bergeron, J., Hasinger, G., et al. 2004, ApJS, 155, 271

Tasca, L. A. M., Le Fevre, O., Ribeiro, B., et al. 2017, A\&A, submitted (arXiv:1602.01842)

Taylor, E. N., Franx, M., van Dokkum, P. G., et al. 2009, ApJS, 183, 295

Townsley, L. K., Broos, P. S., Garmire, G. P., \& Nousek, J. A. 2000, ApJ, 534, L139

Townsley, L. K., Broos, P. S., Nousek, J. A., \& Garmire, G. P. 2002, NIMPA, 486, 751

Treister, E., Virani, S., Gawiser, E., et al. 2009, ApJ, 693, 1713

Vanzella, E., Cristiani, S., Dickinson, M., et al. 2008, A\&A, 478, 83

Vikhlinin, A. 2001, Reducing ACIS Quiescent Background Using Very Faint Mode (Cambridge: CXC) http://cxc.harvard.edu/cal/Acis/Cal_prods/ vfbkgrnd/

Vito, F., Gilli, R., Vignali, C., et al. 2016, MNRAS, 463, 348

Weisskopf, M. C., Gaskin, J., Tananbaum, H., \& Vikhlinin, A. 2015, Proc. SPIE, 9510, 2

Weisskopf, M. C., Tananbaum, H. D., Van Speybroeck, L. P., \& O’Dell, S. L. 2000, Proc. SPIE, 4012, 2

Wuyts, S., van Dokkum, P. G., Franx, M., et al. 2009, ApJ, 706, 885

Xia, L., Malhotra, S., Rhoads, J., et al. 2011, AJ, 141, 64

Xue, Y. Q., Luo, B., Brandt, W. N., et al. 2011, ApJS, 195, 10

Xue, Y. Q., Luo, B., Brandt, W. N., et al. 2016, ApJS, 224, 15

Yang, G., Brandt, W., Luo, B., et al. 2016, ApJ, 831, 145

Young, M., Brandt, W. N., Xue, Y. Q., et al. 2012, ApJ, 748, 124

Zheng, W., Mikles, V. J., Mainieri, V., et al. 2004, ApJS, 155, 73 